Aus der Abteilung Anaesthesiologie

(Prof. Dr. med. M. Quintel)

Im Zentrum Anaesthesiologie, Rettungs- und Intensivmedizin

der Medizinischen Fakultät der Universität Göttingen

\title{
Patientenwege von Patienten mit nicht - spezifischen Rückenschmerzen Eine retrospektive Beobachtungsstudie
}

\author{
INAUGURAL - DISSERTATION \\ zur Erlangung des Doktorgrades \\ der Medizinischen Fakultät \\ der Georg - August - Universität zu Göttingen \\ vorgelegt von \\ Teresa Helbing \\ aus \\ Leinefelde
}

Göttingen 2013 
Dekan: Prof. Dr. rer. nat. H. K. Kroemer

I. Berichterstatter: Prof. Dr. phil. Dipl.-Psych. M. Pfingsten

II. Berichterstatterin: Prof. Dr. E. Hummers- Pradier

Tag der mündlichen Prüfung: 11.03.2014 
$\begin{array}{lr}\text { 1. Einleitung } & 4\end{array}$

2. Wissenschaftlicher Hintergrund $\quad 6$

2.1 Rückenschmerz: Definition (Graduierung) 6

$\begin{array}{lll}2.2 & \text { Epidemiologie und Kosten } & 7\end{array}$

$\begin{array}{lll}2.3 & \text { Verursachung (Klassifikation) } & 10\end{array}$

$\begin{array}{lll}2.4 & \text { Chronifizierung } & 14\end{array}$

2.5 Nationale Versorgungsleitlinie Kreuzschmerz 16

(Version 1.2. August 2011)

$\begin{array}{ll}2.5 .1 \text { Diagnostik } & 16\end{array}$

$\begin{array}{ll}\text { 2.5.1.1 Anamnese } & 16\end{array}$

2.5.1.2 Körperliche Untersuchung, Einstufung 18

$\begin{array}{ll}2.5 .1 .3 \text { Bildgebung } & 18\end{array}$

$\begin{array}{ll}2.5 .2 \text { Therapie } & 19\end{array}$

$\begin{array}{ll}\text { 2.5.2.1 Nichtmedikamentöse Therapie } & 19\end{array}$

2.5.2.2 Medikamentöse Therapie $\quad 22$

2.5.2.3 Invasive Verfahren $\quad 25$

2.5.3 Prävention 25

2.5.4 Multimodale Therapie $\quad 26$

$\begin{array}{ll}2.5 .5 \text { Versorgungskoordination } & 27\end{array}$

2.6 Bisherige Behandlung und Behandlungswege 31

3. Ableitung der Fragestellung/ Problematik der Umsetzung von 35 Leitlinien in der alltäglichen Versorgung 
$\begin{array}{lll}4.1 & \text { Datengenerierung } & 38\end{array}$

4.2 Studienablaufl Patientenrekrutierung 45

$\begin{array}{lll}4.3 & \text { Auswertung } & 47\end{array}$

5. Ergebnisse $\quad 48$

$\begin{array}{lll}5.1 & \text { Patientenprofil } & 48\end{array}$

5.1.1 Alter und BMI $\quad 48$

5.1.2 Soziale Angaben: Personenstand, Schulabschluss, $\quad 49$ berufliche Situation

5.1.3 Laufende Rentenverfahren, MdE, GdB $\mathbf{5 0}$

5.1.4 Rückenschmerzbezogene Krankschreibungen $\mathbf{5 0}$

5.1.5 Schmerzintensität und Chronifizierung (MPSS) $\mathbf{5 0}$

5.1.6 Beeinträchtigungserleben und Depressions-Screening 51 (PDI, ADS)

5.2 Erster Arztkontakt 53

5.2.1 Diagnostische Erstmaßnahmen 53

5.2.1.1 Körperliche Untersuchung 53

5.2.1.2 Bildgebende Diagnostik $\mathbf{5 4}$

5.2.1.2.1 Diagnosemitteilung und Besprechung der Befunde $\quad \mathbf{5 4}$

5.2.2 Berücksichtigung psychosozialer Risikofaktoren 55

5.2.3 Therapeutische Maßnahmen des Erstbehandlers $\quad 57$

5.3 Therapie 58

$\begin{array}{ll}\text { 5.3.1 Medikamente } & \mathbf{5 8}\end{array}$

(einschl. Aufklärung Dosierung, Einnahmedauer, UAW)

5.3.2 Physiotherapie $\quad 60$

5.3.3 Rehabilitationsmaßnahmen $\quad 60$

5.3.4 Operationen $\quad 62$

5.3.5 Weitere Behandlungen insgesamt $\quad 62$ 
5.4 Weiterbehandelnde Arztgruppen neben dem Hausarzt 65

5.4.1. Fachrichtungen $\quad 65$

5.4.1.1 Orthopädie $\quad 66$

5.4.1.2 Neurologie $\quad 67$

5.4.1.3 Neurochirurgie $\quad 68$

5.4.1.4 Sonstige $\quad 69$

5.4.2 Ärztewechsel $\quad 69$

$\begin{array}{ll}5.4 .3 \text { Informationsweitergabe } & \mathbf{7 0}\end{array}$

5.4.3.1 Motivation zu körperlicher Aktivität und Teilnahme an $\quad \mathbf{7 0}$ lokalen Bewegungsprogrammen

5.4.3.2 Vermittlung von Hintergrundwissen/ Edukation $\quad \mathbf{7 0}$

$\begin{array}{ll}\text { 5.4.4 Zufriedenheit mit den behandelnden Arztgruppen } & \mathbf{7 0}\end{array}$

6. Diskussion der Ergebnisse, Vergleich mit der aktuellen Leitlinie 73

6.1 Zusammenfassung der Fragestellung 73

$\begin{array}{lll}6.2 & \text { Patientenbeschreibung } & 76\end{array}$

6.3 Hauptergebnisse der Befragung und Untersuchung 81 auf ihre Leitlinienkonformität

6.4 Beeinträchtigungen der Interpretierbarkeit 100

$\begin{array}{ll}\text { 7. Zusammenfassung } & 103\end{array}$

$\begin{array}{ll}\text { 8. Abkürzungsverzeichnis } & 105\end{array}$

$\begin{array}{ll}\text { 9. Literaturverzeichnis } & 108\end{array}$

$\begin{array}{ll}\text { 10. Abbildungsverzeichnis } & 115\end{array}$

$\begin{array}{ll}\text { 11. Tabellenverzeichnis } & 117\end{array}$ 


\section{Einleitung}

Rückenschmerzen sind einer der häufigsten Anlässe für einen Arztbesuch in Deutschland und verursachen hohe Kosten (s. 2.1; Kohlmann, Schmidt 2005; Schmidt et al. 2007). Die am häufigsten vorzufindenden unkomplizierten Verläufe, bei denen die Beschwerden schnell wieder abklingen, müssen von denen unterschieden werden, die rezidivierend auftreten bzw. in einen chronischen Schmerz münden (siehe Kap. 2.4). Betroffene Patienten haben oft einen langen Leidensweg hinter sich, wobei viele Behandlungsmaßnahmen erfolglos bleiben. Eine der bedeutendsten Folgen der Chronifizierung ist die z.T. lang anhaltende Arbeitsunfähigkeit (Rückenerkrankungen belegen hinter den psychischen Krankheiten und Tumorerkrankungen den Platz 3 der Anlässe für eine Frühberentung (Kohlmann, Schmidt 2005)).

Aufgrund der Vielfalt an angebotenen Behandlungsmethoden, die häufig ohne Effekt bleiben, wurde im Jahr 2005 von der Bundesärztekammer eine Nationale Versorgungsleitlinie in Auftrag gegeben (siehe Kap. 2.5). Darin werden diagnostische und therapeutische Verfahren bzgl. ihrer Evidenz beurteilt und Patientenwege vorgeschlagen, die sich weitgehend am Verlauf der Rückenschmerzen orientieren (akut-subakut-chronisch). Die für die Behandlung von Rückenschmerzen vorliegenden Leitlinien (siehe. Kap. 2.5) unterscheiden sich in ihrer grundsätzlichen Ausrichtung nicht. Im Grunde gehen die Empfehlungen daher seit Jahren in eine einheitliche Richtung, die durch die Abkehr von passiven Maßnahmen, eine Hinwendung zu Beratung und Aktivierung sowie möglichst wenig Invasivität der Behandlungen gekennzeichnet ist.

Insofern liegt es nahe, zu prüfen, ob diese grundsätzlichen diagnostischen und therapeutischen Orientierungen im Versorgungsalltag eingehalten werden. Hierbei ist besonders zu beachten, dass durch bestimmte Abläufe (z.B. Umgang mit Bildgebung) Chronifizierungen sogar gefördert werden können. In dieser Studie beschäftigen wir uns daher mit der Frage, wie Patienten, die an bereits chronifizierten Rückenschmerzen leiden, in ihrem Krankheitsverlauf behandelt wurden.

Die Datengrundlage stellte die Auswertung von Arztbriefen, Befundberichten, Ergebnissen des Deutschen Schmerzfragebogens (DSF 2012) sowie Ergebnissen 
unserer standardisierten Patientenbefragung bei einem retrospektivem Befragungszeitraum von 3 Jahren dar.

Ziel unserer Studie war es, die Behandlungsvorgeschichte chronischer Rückenschmerz-Patienten systematisch bis zur Vorstellung in einer spezialisierten Schmerzklinik (Schmerztagesklinik und -ambulanz der Universitätsmedizin Göttingen) zu erfassen, zu analysieren und auf ihre Leitlinien-Kompatibilität zu prüfen, um die dadurch gewonnenen Erkenntnisse bezüglich möglicher Schwachstellen im Versorgungssystem für eine Verbesserung und Beschleunigung der Behandlungsabläufe zu nutzen. 


\section{Wissenschaftlicher Hintergrund}

\subsection{Rückenschmerz: Definition (Graduierung)}

Eine Definition von Rückenschmerzen ist dadurch erschwert, dass sie umgangssprachlich bzgl. der Lokalisation den gesamten Rücken umfassen. Im medizinischen Sprachgebrauch ist jedoch mindestens die Unterteilung in die Bereiche HWS/obere BWS, BWS und LWS zu unterscheiden. Der Bereich der Lendenwirbelsäule (mit oder ohne Ausstrahlung der Schmerzen) macht die meisten Fälle aus und steht auch wissenschaftlich im Fokus des Interesses der letzten Jahre. Eine präzise Definition müsste dementsprechend lauten: Schmerzen im Bereich der (unteren) Lendenwirbelsäule. Im Wording von modernen Leitlinien, wie der „Nationalen Versorgungsleitlinie Kreuzschmerz" (Version 1.2., 2011) oder der DEGAM-Leitlinie Nr. 3: Kreuzschmerzen (2003), wird dieser Bereich mit dem Begriff „Kreuzschmerz" bezeichnet.

Unter Kreuzschmerzen versteht man laut ICD-10 (WHO, International Classification of Diseases, 2012) (M 54.5) Schmerzen im Bereich des Rückens vom unteren Rippenbogen bis zu den Glutäalfalten, die mit einer Ausstrahlung in die Beine einhergehen können (Abb.1).

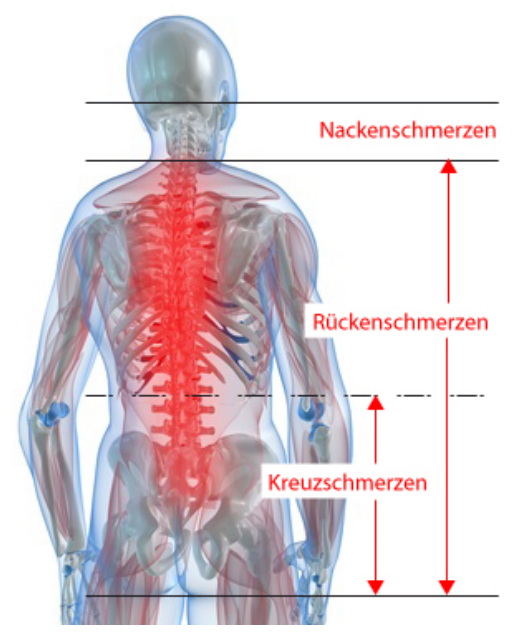

Abb. 1

Lokalisation von: Nackenschmerzen - Rückenschmerzen - Kreuzschmerzen http://www.kreuzschmerzen-rueckenschmerzen.de/ (2011) 
Neben der Lokalisation ist die zeitliche Dimension ein weiteres Differenzierungsmerkmal:

- Als akute Kreuzschmerzen bezeichnet man Schmerzepisoden von weniger als 12 Wochen Dauer. Die Schmerzintensität kann während dieses Zeitraumes variieren.

- Bestehen die akuten Beschwerden länger als 6 Wochen, bezeichnet man sie als "subakut".

- Treten die Kreuzschmerzen nach einem symptomfreien Intervall von mindestens 6 Monaten wieder auf, spricht man von rezidivierenden akuten Kreuzschmerzen.

- Als chronisch sind die Schmerzen anzusehen, wenn sie mindestens 12 Wochen oder länger bestehen (DEGAM-Leitlinie Nr. 3: Kreuzschmerzen 2003).

„Der zugrunde liegende Pathomechanismus reicht von (seltenen) spezifischen Ursachen mit erheblichen Beschwerden bis zu (unbedenklichen) Belastungsschmerzen als Zeichen körperlicher Beanspruchung bei mangelnder Fitness." (Pfingsten, Hildebrandt 2010, S. 1) Der Mehrzahl der Erkrankungsfälle liegen keine spezifischen Krankheitsprozesse zu Grunde. Oft handelt es sich um sogenannte nicht-spezifische Rückenschmerzen (Pfingsten, Hildebrandt 2010; siehe Kapitel 2.3 (S.10)).

Laut Pfingsten \& Hildebrandt (2010) fehlt eine klar abgrenzbare Krankheitsentität auf Grund der Vielzahl möglicher Symptome sowie zahlreicher unterschiedlicher Entstehungsmechanismen.

\subsection{Epidemiologie und Kosten}

Bzgl. der epidemiologischen Angaben rangieren Rückenschmerzen meist sehr oft auf den ersten Plätzen (Kohlmann, Schmidt 2005).

"Es gibt kaum ein Krankheitsbild, das in Bezug auf Prävalenz- und Inzidenzraten (Inzidenz 2\%) sowie in Bezug auf die entstehenden Kosten eine derart ansteigende Tendenz aufweist wie muskuloskelettale Beschwerden und insbesondere Rückenschmerzen." (Pfingsten, Hildebrandt 2010, S. 1). Verglichen mit anderen Erkrankungen lässt sich ein „unvermindert aufwärts steigender Trend hinsichtlich 
Behandlungszahlen und Invaliditätsraten" beobachten (Hildebrandt, Mense 2001, S. 411).

Epidemiologischen Schätzungen zufolge leiden ca. 85\% der Bevölkerung westlicher Industriestaaten mindestens einmal in ihrem Leben an Rückenbeschwerden. Dabei beträgt die Punktprävalenz bis zu 40\% (Hildebrandt, Mense 2001).

Laut Becker et al. (2003) sowie Kohlmann \& Schmidt (2005) findet man bei niedergelassenen Ärzten eine Praxisprävalenz von 6-8\% pro Woche (in DEGAMLeitlinie Nr. 3: Kreuzschmerzen (2003)).

Insgesamt sind Schmerzsymptome in etwa $20 \%$ der Fälle der Anlass für die Inanspruchnahme ärztlicher Primärversorgung. Davon belegen Rückenschmerzen mit ca. 48\% eindeutig Platz 1 (Kohlmann, Schmidt 2005).

Häufigste Lokalisation mit ca. 18\% ist die Lendenwirbelsäule (Pfingsten, Hildebrandt 2010). Frauen sind deutlich häufiger betroffen als Männer. Verantwortlich für diese erhöhte Symptombelastung ist laut Kohlmann \& Schmidt (2005) „ein komplexes Zusammenspiel von biologischen, psychischen und sozialen Faktoren" (Kohlmann, Schmidt 2005, S. 2).

Laut Pfingsten \& Hildebrandt (2010) ist es wichtig, eine Unterscheidung zwischen degenerativen Altersveränderungen der Wirbelsäule (die im Laufe des Lebens bei nahezu allen Menschen auftreten) und schmerzhaften Rückenveränderungen mit eindeutiger körperlicher Pathologie zu treffen. Während degenerative Veränderungen der Wirbelsäule oft in der 3. Lebensdekade beginnen und im Altersverlauf einen ständigen Zuwachs zeigen, treten Rückenschmerzen zwar sehr häufig in der Altersspanne um 40 Jahre herum auf, danach sinkt ihre Inzidenz jedoch stetig ab.

Obwohl Rückenschmerzen häufig zwischen der 3. und 5. Lebensdekade auftreten, kann nicht von einem (einzigen) Schwerpunkt in dieser Lebensspanne gesprochen werden, es existiert eine breite Streuung der Schweregrade. Dass der zeitliche Schwerpunkt von Rückenschmerzen oft im mittleren Lebensalter gesehen wird, lässt sich laut Pfingsten \& Hildebrandt (2010) dadurch erklären, dass in dieser Altersspanne „die Folgen der Erkrankung (Arbeitsunfähigkeit) besonders ins Gewicht fallen“ (S. 2) und die gesellschaftliche Relevanz daher in dieser Spanne am höchsten ist. Denn die „sozialmedizinisch und volkswirtschaftlich bedeutsamste Folge von Rückenschmerzen 
ist die zeitlich befristete Arbeitsunfähigkeit. Im statistischen Durchschnitt verliert jedes Pflichtmitglied der gesetzlichen Krankenversicherung in Deutschland pro Jahr etwa 2 bis 3,5 Arbeitstage aufgrund von Rückenschmerzen." (Kohlmann, Schmidt 2005, S. 5)

Betrachtet man die Frühberentungen wegen verminderter Erwerbsfähigkeit, so war dafür im Jahr 2002 in 14,5\% der Fälle bei den Männern eine Dorsopathie ursächlich (bei den Frauen in 11,1 \% der Fälle). Damit rangierten die Rückenerkrankungen hinter den psychischen Krankheiten und Tumorerkrankungen auf Platz 3 der Anlässe für eine Frühberentung und lagen somit noch vor den Herz-Kreislauf-Erkrankungen.

Die Bedeutung der Frühberentungsproblematik spiegelt sich verständlicherweise auch in den resultierenden Kosten wider. Davon entfallen lediglich $30 \%$ auf die direkten Kosten (medizinische Behandlung), wohingegen die indirekten Kosten, zu denen man z.B. die durch Produktivitätsausfälle bedingten Kosten zählt, wie Berentung und Arbeitsunfähigkeit, 70\% der Gesamtausgaben betragen (Kohlmann, Schmidt 2005).

Insgesamt ist die Behandlung von Rückenschmerzen kostenintensiv. Rückenschmerzen zählen zu den sieben teuersten Erkrankungen in Deutschland (Becker et al. 2010, Statistisches Bundesamt 2012). Für Kohlmann \& Schmidt (2005) stehen die Behandlungskosten von Rückenschmerzen unter allen Krankheitsgruppen sogar an erster Stelle. Insgesamt betragen die Ausgaben fast 50 Mrd. Euro pro Jahr, machen 2,2\% des Bruttoinlandproduktes aus (Pfingsten, Hildebrandt 2010) und laut Kohlmann \& Schmidt (2005) ca. 1\% des Bruttosozialproduktes.

5\% aller Fälle sind sogenannte Problemfälle, das heißt, für diese kleine, aber dennoch sehr kostenintensive Patientengruppe gibt es derzeit „keine adäquaten Behandlungsmöglichkeiten" (Hildebrandt, Mense 2001, S. 411). Diese Problemfälle verursachen laut Hildebrandt \& Mense (2001) jedoch die meisten Kosten, nämlich ca. 50\% der Gesamtkosten. Auch die Studie von Becker et al. (2010) bestätigt: die Kosten für chronische Rückenschmerzpatienten sind doppelt so hoch wie für akute Kreuzschmerzen. Als wichtige Kosten-Prädiktoren gelten der Studie zufolge die Bewertung und Einschätzung der Patienten bezüglich ihrer Rückkehr zum Arbeitsplatz sowie weitere psychosoziale Einflussfaktoren (wie z.B. das Vorhandensein von Depressionen). 


\subsection{Verursachung (Klassifikation)}

Die Definition und Beschreibung der Pathogenese von Rückenschmerzen ist problematisch. Pathogenetisch betrachtet, muss die überwiegende Mehrzahl aller Rückenschmerzen (ca. 85\%) als nicht-spezifischer Rückenschmerz beurteilt werden (Pfingsten, Hildebrandt 2010). Dieser Begriff wurde erstmals vom schottischen Orthopäden Gordon Waddell benutzt und bedeutet, dass die genaue Angabe einer Ursache zur Erklärung der Schmerzen im pathologischen Sinne nicht möglich ist (Hildebrandt, Pfingsten 2000).

Dennoch gibt es natürlich eindeutige somatische Auslöser von Rückenschmerzen: neben sehr selten auftretenden infektiösen (z.B. Diszitis) und seltenen entzündlichen rheumatischen Erkrankungen (z.B. M. Bechterew, Reiter-Syndrom) können anhaltende Rückenschmerzen in ca. 0,7\% als Symptom oder als in den Bereich der Wirbelsäule projizierte Schmerzen durch neoplastische Prozesse sowie eine Reihe von gynäkologischen, urologischen und internistischen Erkrankungen (z.B. Endometriose, Nephrolithiasis, Pankreaskarzinom) hervorgerufen werden.

Laut Pfingsten \& Hildebrandt (2010) lassen sich bei den spezifischen Rückenschmerzen 2 Arten von Schmerzen unterscheiden: radikuläre und nichtradikuläre Schmerzen.

Radikuläre Schmerzen (welche in der DEGAM-Leitlinie Nr. 3: Kreuzschmerzen 2003 auch als Lumboischialgie bezeichnet werden) beinhalten z.B. durch Bandscheibenvorfälle verursachte Rückenschmerzen. Hierbei kommt es zu einer „mechanischen Kompression bzw. Dehnung der Nervenwurzel durch protrahiertes Bandscheibenmaterial“. Dieser Wurzelkompression folgt eine lokale Entzündung und Schwellung der betroffenen Wurzel. Manchmal können kleine Bandscheibenvorfälle „erhebliche Schmerzen“ verursachen, andererseits können auch größere Bandscheibenvorfälle symptomlos bleiben. Kriterien eines bandscheibenbedingten radikulären Schmerzes sind zum einen Schmerzen im Bein (einschließlich Gesäß), die jedoch stärker sind als die Schmerzen im Rücken. Weiterhin findet man oft Sensibilitätsstörungen des betroffenen zugehörigen Dermatoms, eine Parese der entsprechenden Kennmuskulatur (Pfingsten, Hildebrandt 2010) sowie eine Ausstrahlung in den Fuß oder die Zehen. Reflexauffälligkeiten und motorische oder 
sensible Ausfälle im Versorgungsgebiet einer Nervenwurzel sind keine Seltenheit. (DEGAM-Leitlinie Nr. 3: Kreuzschmerzen 2003); ein wichtiges diagnostisches Zeichen ist der Lasègue-Test (Abb. 2), wenn er einen Wert $<50 \%$ des normalen anderen Beines erbringt.

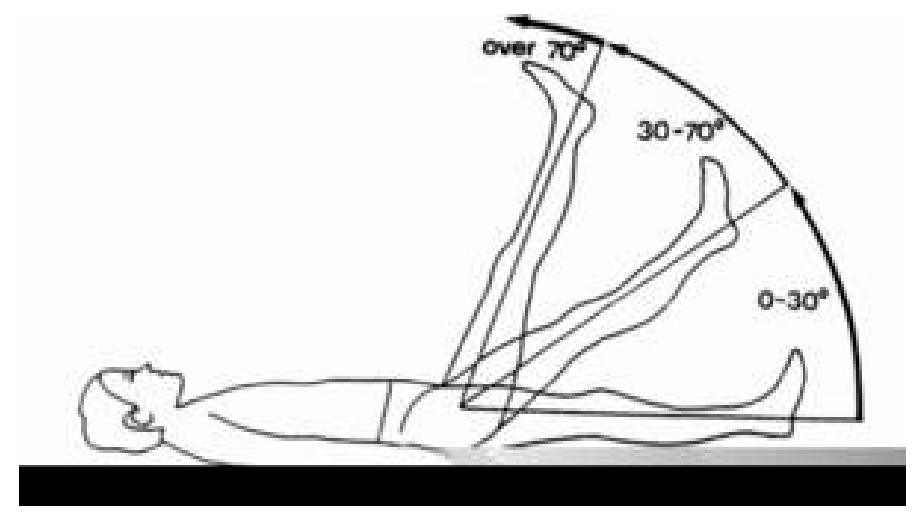

Abb. 2

Lasègue-Test

http://www.erste-hilfe-infos.de/index.php?id=1505 (2011)

„Abgesehen von Bandscheibenvorfällen können radikuläre Schmerzen auch durch knöcherne Irritationen bzw. eine Engpasssituation (Stenose) im Verlauf des Spinalnervs verursacht werden" (Pfingsten, Hildebrandt 2010, S. 5). Die typische Beschwerdesymptomatik äußert sich durch eine sog. neurogene Claudicatio, das bedeutet, dass die Patienten schon nach einer sehr kurzen Gehstrecke (50-100m) wegen zunehmender Schmerzen oder Schwäche in den Beinen stehen bleiben müssen. Dies hält nur wenige Minuten an, danach verschwindet die Symptomatik wieder. Typisch für Patienten mit spinaler Stenose ist eine Abnahme der Beschwerden beim Fahrradfahren. Außerdem sind die neurologischen Symptome eher „flüchtig und nicht so manifest wie bei Bandscheibenvorfällen" (Pfingsten, Hildebrandt 2010, S. 5). Ein weiterer Grund für radikuläre Beschwerden kann auch eine Spondylolisthese (Wirbelgleiten, angeboren oder erworben) sein, die jedoch oft symptomlos bleibt. 
Hierbei führt eine Nervenwurzel über eine entstandene Knochenstufe und wird bei Bewegung gedehnt.

Wie bereits oben ausgeführt, ist eine einzige konkrete Ursache der Beschwerden jedoch eher selten zu finden. In der Mehrzahl der Fälle wird man mit einer Kombination verschiedener Störungen konfrontiert (z.B. eine zentrale Bandscheibenprotrusion und eine laterale Stenose oder eine laterale Bandscheibenprotrusion und eine laterale Stenose, die beide für sich allein keine Beschwerden hervorrufen würden, aber in Kombination doch zu radikulären Schmerzen führen) (Pfingsten, Hildebrandt 2010).

Die zweite große Gruppe der Schmerzen sind die nicht-radikulären Beschwerden. Diese sind „dumpf, tiefsitzend, schlecht lokalisierbar und können nach proximal oder weit distal ausstrahlen, ohne dass ein eindeutiger pathologischer Befund zu erheben ist" (Pfingsten, Hildebrandt 2010, S. 5). Auch sind sie deutlich häufiger als radikulär bedingte Schmerzen anzutreffen. „Sie können von vorderen Anteilen der Wirbelsäule (Bandscheiben) oder hinteren Strukturen (Gelenken, Muskeln, Bändern) ausgehen." (Pfingsten, Hildebrandt 2010, S. 5). Es lässt sich erkennen, dass die genauen Ursachen dieser Schmerzen "wesentlich schwieriger zu diagnostizieren" sind (Pfingsten, Hildebrandt 2010, S. 5).

Wenn z.B. der hintere Bereich des Anulus fibrosus der Bandscheiben (der viele Schmerzrezeptoren besitzt) durch Degeneration gereizt wird, kann es zu sog. diskogenen Schmerzen kommen.

Außerdem kann eine segmentale Instabilität zu nicht-radikulären Schmerzen führen, die vor allem im Tagesverlauf mit zunehmender Belastung (v. a. nach längerem Sitzen und Stehen) ansteigen. Sie bessern sich deutlich im Liegen.

Auch sind die hinten liegenden Zwischenwirbelgelenke häufig in den degenerativen Prozess einbezogen. "Möglicherweise kommt es im Gefolge der Bandscheibendegeneration mit Funktionsstörungen im Bewegungssegment zu einer zunehmenden Irritation und zum Teil zur Degeneration dieser Gelenke. Die Beschwerden werden in der Regel im Bereich des Rückens, des Gesäßes und der Hinterseite der Oberschenkel empfunden, manchmal auch in der Leiste und seltener im Unterschenkel und Fuß." (Pfingsten, Hildebrandt 2010, S. 6) Typisch hierfür sind sog. 
Anlaufschmerzen morgens nach dem Aufstehen, die im Tagesverlauf abnehmen. „Die Schmerzen verstärken sich bei Lagewechsel und wenn längere Zeit eine eintönige Haltung eingenommen wird sowie bei der Reklination und nach dem Wiederaufrichten aus nach vorn gebückter Haltung." (Pfingsten, Hildebrandt 2010, S. 6) Leider ist eine Abgrenzung zu diskogenen oder muskulären Beschwerden schwer möglich.

Weiterhin zählt man funktionelle Störungen der lliosakralgelenke zu den nichtradikulären Beschwerden. „Störungen im Bereich dieser Gelenke gehen mit lokalisiertem Schmerz im Bereich der unteren Wirbelsäule sowie einer Ausstrahlung in die untere Extremität (Gesäß, dorsolateraler Oberschenkel, Wade, laterale Ferse) einher und können akut auftreten (bei Bewegungen, die eine Rumpfbeugung mit gleichzeitiger Rotation und Beckenkippung beinhalten, oder bei einem Sturz sowie während der Schwangerschaft) oder sich langsam einstellen (oft begleitend bei Bandscheibenerkrankungen bzw. nach Bandscheibenoperationen)." (Pfingsten, Hildebrandt 2010, S. 6)

Insgesamt kommt es oft zu einer additiven Wirkung verschiedener Verursachungsmechanismen: Eine ständige Überlastung der Wirbelsäule kann z.B. zu Koordinationsstörungen und Fehlinnervationen der Muskulatur führen, die dadurch degenerative Veränderungen in den Bereichen der Bewegungssegmente beschleunigen können. Diese segmentale Störung im Bereich der Wirbelsäule (z.B. Blockierung bzw. Fehlstellung der Gelenke oder Degeneration einer Bandscheibe) kann dann neben einer unmittelbaren Reizung der Schmerzrezeptoren eine reflektorische Muskelverspannung (sog. spondylogenes Reflexsyndrom) zur Folge haben. „Diese reflektorisch bedingte Muskelspannung führt über spezielle physiologische Prozesse zu einer erneuten Nozizeption. " (Pfingsten, Hildebrandt 2010, S. 6)

Ein wichtiger Punkt betrifft auch psychische Spannungen, die sich auf die Muskulatur übertragen können, „da das $\gamma$-System (dessen Ausgangsort die Muskelspindeln sind) einer supraspinalen Kontrolle (besonders der Formatio reticularis) untersteht". (Pfingsten, Hildebrandt, S. 6). Da in der Nackenmuskulatur und im M. erector trunci besonders viele Muskelspindeln enthalten sind, ist es naheliegend und nachvollziehbar, 
dass sich psychische Spannungen häufig in Form von Schulter-, Nacken-, Kopf- bzw. Kreuzschmerzen manifestieren (Pfingsten, Hildebrandt 2010).

\subsection{Chronifizierung}

Bei ca. 35\% aller Betroffenen mit Rückenschmerzen lässt sich eine Chronifizierungstendenz beobachten (Mesrian et al. 2005). Unter dem Begriff der yellow flags fasst man eine Vielzahl Chronifizierungs-beschleunigenden Faktoren zusammen. Ein erhöhtes Alter zu Krankheitsbeginn sowie bereits vorliegende degenerative Prozesse, wie z.B. Osteophyten oder Sklerosierungen, können sich ebenso negativ auf den Krankheitsverlauf auswirken wie beispielsweise ein generell ungesunder Lebensstil (Rauchen, Adipositas, geringe körperliche Fitness, mangelnde Bewegung) (Handlungsleitlinie Kreuzschmerzen 3. Auflage, Arzneimittelkommission der deutschen Ärzteschaft 2007).

Die vielfältig dazu vorliegende Literatur zeigt, dass das erstmalige Auftreten der Beschwerden oftmals eher durch exogene Faktoren ausgelöst wird (z.B. die körperliche Belastung am Arbeitsplatz), während bei Rezidiven und der Chronifizierung vor allem psychosoziale Variablen (z.B. Krankheitsverarbeitung) eine wichtige Rolle spielen (Pfingsten, Hildebrandt 2010).

Schon- und Vermeidungsverhalten, falsche Krankheitsmodellvorstellungen, eine Fixierung auf Schaden mit selbstständiger Initiierung vielfältiger diagnostischer Maßnahmen (z.B. sog. Ärzte-Hopping) und psychosoziale Beeinträchtigungen sind Faktoren auf Seiten der Patienten, die als negative Prädiktoren für einen chronischen Krankheitsverlauf bekannt sind (Müller, Pfingsten 2004).

Sehr große Bedeutung kommt demnach der frühzeitigen Erfassung möglicher psychischer Einflussfaktoren zu (siehe auch Kapitel 2.5.4 (S. 26), 2.5 .5 (S. 27) und 5.2.2 (S. 55)). Hierzu zählt man z.B. eine pessimistische Lebenseinstellung gekoppelt mit einem "starken Krankheitsgefühl und Schmerzerleben" (DEGAM-Leitlinie Nr. 3: Kreuzschmerzen 2003, S.7) sowie „Defizite bezüglich assertiver Kompetenzen (Selbstsicherheit, Selbstbehauptung)“ (Handlungsleitlinie Kreuzschmerzen 3. Auflage, Arzneimittelkommission der deutschen Ärzteschaft 2007, S. 1) und „emotionale 
Beeinträchtigungen“ wie z.B. Depressionen und Angststörungen (Handlungsleitlinie Kreuzschmerzen 3. Auflage, Arzneimittelkommission der deutschen Ärzteschaft 2007, S. 1).

Des Weiteren sind Arbeitsunfähigkeitszeiten von mehr als 4-6 Wochen (Von Korff et al. 1993 in: DEGAM-Leitlinie Nr. 3: Kreuzschmerzen 2003) sowie ein unsicherer Arbeitsplatz bzw. Unzufriedenheit mit der Arbeit und ein Rentenwunsch eher hinderlich bezüglich eines positiven Outcome. Auch bereits rezidivierende Schmerzattacken sind eher als negativer Prädiktor zu werten (DEGAM-Leitlinie Nr. 3: Kreuzschmerzen 2003). Es ist wichtig zu unterscheiden „zwischen den Mechanismen, die Rückenschmerzen auslösen können, und solchen, die für seine Aufrechterhaltung verantwortlich sind" (Pfingsten, Hildebrandt 2010, S. 9).

Auch sog. iatrogene Faktoren können an der Aufrechterhaltung von Rückenschmerzen oder ihrer Chronifizierung beteiligt sein: dazu gehören z.B. die fehlende Berücksichtigung psychosozialer Risikofaktoren durch den Arzt, eine inadäquate Kommunikation des radiologischen Ergebnisses oder die fehlende Motivation zur körperlichen Aktivität (Müller, Pfingsten 2004). 


\subsection{Nationale Versorgungsleitlinie Kreuzschmerz \\ (Version 1.2. August 2011)}

\subsubsection{Diagnostik}

\subsubsection{Anamnese}

Die Basis für die Behandlung von Kreuzschmerzen ist eine sorgfältige Anamnese, die sowohl „biologische, psychische als auch soziale Aspekte“ einbezieht (Nationale Versorgungsleitlinie Kreuzschmerz, Version 1.2., 2011, S. 45). Erfragt werden sollten jegliche Begleitsymptome und Vorerkrankungen, die richtungweisend für eine dringliche Behandlung aufgrund spezifischer Krankheitsursachen sein können. Um die im Abschnitt 2.3 genannten ernsten Erkrankungen (auf traumatischer, tumoröser, radikulärer oder infektiöser Basis) als Ursache der Rückenschmerzen zu identifizieren, sollten die sogenannten red flags (s. Tab. 1) als spezifische Warnsignale genau erhoben werden. Als Frakturhinweise dienen z.B. Traumata in der Anamnese, eine Osteoporose-Erkrankung oder eine kürzlich stattgefundene Steroidtherapie. Als Tumorzeichen gelten Gewichtsverlust, Appetitlosigkeit, rasche Ermüdbarkeit und nächtliche Schmerzen. Möglicherweise besteht sogar bereits ein Tumorleiden in der Vorgeschichte. Auch sollte erfragt werden, ob im Liegen eine Schmerzverstärkung auftritt, ob Probleme beim Wasserlassen bestehen (z.B. Harnverhalt oder Inkontinenz) oder ob fortschreitende neurologische Ausfälle beobachtet wurden (z.B. Gefühlsstörungen wie Taubheitsgefühle, Schwäche und Kribbelparästhesien oder eine Lähmung der unteren Extremität, wie z.B. eine Fußheberschwäche) (Pfingsten, Hildebrandt 2010). Weiterhin sollte erhoben werden, ob kürzlich Fieber oder Schüttelfrost bestanden. Außerdem können ein sehr junges Alter zum Zeitpunkt der Beschwerden (< 20 Jahre) und ein „zunehmender, nicht bewegungsabhängiger Schmerz oder eine Persistenz der Beschwerden trotz Therapie“" (DEGAM-Leitlinie Nr. 3: Kreuzschmerzen 2003, S. 8) richtungweisend sein. Mit zunehmender Anzahl der genannten Warnhinweise steigt die Wahrscheinlichkeit, dass die Symptome einen eher schwerwiegenden Verlauf nehmen. Jedoch erlaubt erst das Gesamtbild aller Symptome 
eine Aussagekraft und die Einschätzung des individuellen Risikos des Patienten (DEGAM-Leitlinie Nr. 3: Kreuzschmerzen 2003).

\begin{tabular}{|c|c|c|c|}
\hline Fraktur & Tumor & Infektion & Radikulopathien \\
\hline $\begin{array}{l}\text { • schwerwiegendes } \\
\text { Trauma z. B. durch } \\
\text { Autounfall oder Sturz } \\
\text { aus größerer Höhe, } \\
\text { Sportunfall } \\
\text { - Bagatelltrauma (z. B. } \\
\text { Husten, Niesen oder } \\
\text { schweres Heben bei } \\
\text { älteren oder } \\
\text { potentiellen } \\
\text { Osteoporose-patienten) } \\
\text { • systemische } \\
\text { Steroidtherapie }\end{array}$ & $\begin{array}{l}\text { • höheres Alter } \\
\text { • Tumorleiden in der } \\
\text { Vorgeschichte } \\
\text { • allgemeine } \\
\text { Symptome: } \\
\text { Gewichtsverlust, } \\
\text { Appetitlosigkeit, rasche } \\
\text { Ermüdbarkeit } \\
\text { • Schmerz, der in } \\
\text { Rückenlage zunimmt } \\
\text { • starker nächtlicher } \\
\text { Schmerz }\end{array}$ & $\begin{array}{l}\text { • allgemeine Symptome, } \\
\text { wie kürzlich aufgetretenes } \\
\text { Fieber oder Schüttelfrost, } \\
\text { Appetitlosigkeit, rasche } \\
\text { Ermüdbarkeit. } \\
\text { • durchgemachte } \\
\text { bakterielle Infektion } \\
\text { • i.v.-Drogenabusus } \\
\text { •Immunsuppression } \\
\text { • konsumierende } \\
\text { Grunderkrankungen } \\
\text { • kürzlich zurückliegende } \\
\text { Infiltrationsbehandlung an } \\
\text { der Wirbelsäule } \\
\text { • starker nächtlicher } \\
\text { Schmerz }\end{array}$ & $\begin{array}{l}\text { • straßenförmig in ein oder } \\
\text { beide Beine ausstrahlende } \\
\text { Schmerzen, ggf. verbunden } \\
\text { mit Gefühlsstörungen wie } \\
\text { Taubheitsgefühlen oder } \\
\text { Kribbelparästhesien im } \\
\text { Schmerzausbreitungsgebiet } \\
\text { oder Schwächegefühl } \\
\text { • Kaudasyndrom: } \\
\text { - plötzlich einsetzende Blasen- } \\
\text { Mastdarmstörung, z. B. } \\
\text { Urinverhalt, vermehrtes } \\
\text { Wasserlassen, Inkontinenz } \\
\text { - Gefühlsstörung } \\
\text { perianal/perineal } \\
\text { • Ausgeprägtes oder } \\
\text { zunehmendes neurologisches } \\
\text { Defizit (Lähmung, } \\
\text { Sensibilitätsstörung) der } \\
\text { unteren Extremität } \\
\text { • Nachlassen des Schmerzes } \\
\text { und zunehmende Lähmung bis } \\
\text { zum kompletten Funk- } \\
\text { tionsverlust des Kennmuskels } \\
\text { (Nervenwurzeltod) }\end{array}$ \\
\hline
\end{tabular}

Tab. 1

Warnhinweise auf eine spezifische vertebragene Ursache („red flags“)

(Nationale Versorgungsleitlinie Kreuzschmerz - Kurzfassung.

Version 1.2. August 2011) 


\subsubsection{Körperliche Untersuchung, Einstufung}

Ist aufgrund der anamnestischen Erhebungen ein Trauma, ein Tumorleiden, ein entzündlicher Prozess oder ein Kaudasyndrom naheliegend, so ist diesen selbstverständlich weiter diagnostisch nachzugehen.

Liegen Hinweise für Erkrankungen anderer Organsysteme vor, soll sich eine Untersuchung dieser Regionen anschließen.

Findet man anamnestisch keine red flags, so wird eine generelle körperliche, klinische Basisuntersuchung empfohlen.

Liegt auch nach der klinischen Untersuchung kein Hinweis auf einen akuten gefährlichen Verlauf und schwerwiegende Erkrankungen vor, werden die Schmerzen als nicht-spezifischer Kreuzschmerz eingeordnet und es soll vorerst auf weitere diagnostische Maßnahmen (wie Röntgen etc.) verzichtet werden.

\subsubsection{Bildgebung}

Laut „Nationaler Versorgungsleitlinie Kreuzschmerz" (Version 1.2., 2011) ist eine routinemäßige Bildgebung obsolet.

Liegen red flags vor, so wird eine bildgebende Untersuchung betreffend der Verdachtsdiagnose empfohlen.

Bei einem akuten Kreuzschmerz, bei dem nach Anamnese und körperlicher Untersuchung ein schwerwiegender Verlauf weitestgehend ausgeschlossen wurde, soll vorerst keine Bildgebung erfolgen.

Für Rückenschmerzen, die nach 6-wöchiger leitlinienorientierter Behandlung keine Besserung und evtl. sogar eine Progression zeigen, wird eine einmalige bildgebende Diagnostik empfohlen. 


\subsubsection{Therapie}

\subsubsection{Nichtmedikamentöse Therapie}

Generell ist laut der „Nationalen Versorgungsleitlinie Kreuzschmerz" (Version 1.2., 2011) bei akutem Kreuzschmerz die Vermeidung unnötiger Medikation und die Konzentration auf aktivierende Maßnahmen wie die Vermeidung passiver Methoden wünschenswert.

\section{- Akupunktur}

Da bei dieser Art der Behandlung keine Aktivierung des Patienten erfolgt, wird eine Akupunkturbehandlung bei akutem Rückenschmerz nicht empfohlen. Bei einer durchschnittlichen Anzahl von 6-10 Sitzungen ist diese Art der Therapie bei einem Krankheitsbild mit zügiger Besserungstendenz nicht sinnvoll.

Auch bei chronischem Kreuzschmerz zeigt die vorliegende Evidenz, dass die Anwendung von Akupunktur nur eingeschränkt hilfreich ist. Zum einen wird dadurch keine Selbstaktivierung des Patienten erreicht, zum anderen unterliegt der Therapieeffekt einer großen Breite aufgrund unterschiedlichster Therapiequalitäten und -standards.

\section{- Bettruhe}

Akuter nicht-spezifischer Kreuzschmerz soll nicht mit Bettruhe behandelt werden. Natürlich kann die Schwere der Schmerzen eine kurzfristige Bettruhe erforderlich machen. Um eine Chronifizierung zu verhindern, wird jedoch eine zügige Rückkehr zu den gewohnten Aktivitäten angestrebt.

Auch bei chronischem Rückenschmerz wird von Bettruhe abgeraten, da diese die Rehabilitation erschwert und das „passive Krankheitsverhalten“ (Nationale Versorgungsleitlinie Kreuzschmerz" Version 1.2., 2011, S. 23) verstärkt. 


\section{- Bewegung}

Generell sollen die Patienten zu körperlicher Aktivität motiviert werden. Dennoch sollen Therapieverfahren wie Krankengymnastik nicht regulär zur Therapie des akuten nichtspezifischen Kreuzschmerzes verschrieben werden und auch bei chronischem Rückenschmerz sollen Bewegungstherapien nicht die primäre Behandlung darstellen.

\section{- Elektrotherapie}

Hierzu zählen die Interferenztherapie, die transkutane elektrische Nervenstimulation (TENS) und die perkutane elektrische Nervenstimulation (PENS). Unsere Untersuchungen beziehen sich jedoch ausschließlich auf die TENS.

Sowohl akuter nicht-spezifischer Kreuzschmerz als auch chronischer Rückenschmerz soll laut der „Nationalen Versorgungsleitlinie Kreuzschmerz“ (Version 1.2., 2011) nicht mit TENS behandelt werden. Es fördert die unerwünschte Passivität des Patienten und wird bisher von keinem Wirksamkeitsnachweis gestützt.

\section{- Entspannungsverfahren}

Da Entspannungstechniken einen gewissen Zeitraum beanspruchen, bis sie erlernt sind, werden solche Verfahren nur eingeschränkt beim akuten nicht-spezifischen Kreuzschmerz eingesetzt. Dennoch gibt es Evidenz dafür, dass sie, in einem biopsycho-sozialen Kontext angewendet, einer Chronifizierung vorbeugen können.

Das Entspannungsverfahren Progressive Muskelrelaxation (PMR) wird somit bei erhöhtem Chronifizierungspotential akuter Patienten sowie bei chronischem nichtspezifischen Kreuzschmerz empfohlen.

\section{- Manipulationen/ Mobilisation}

Sowohl bei akutem als auch bei chronischem nicht-spezifischen Rückenschmerz kann eine Manipulations- bzw. Mobilisationsbehandlung in die Therapie einbezogen werden (bei chronischem Kreuzschmerz in Verbindung mit einer Bewegungstherapie). 
- Massagen

Da Massagen die Passivität des Patienten unterstützen bzw. inn möglicherweise nicht zu ausreichender Eigenaktivität motivieren, wird diese Behandlungsform für akute Kreuzschmerz-Patienten nicht empfohlen. Bei chronischem Rückenschmerz kann eine Kombination aus Massage und Bewegungstherapie erfolgen.

\section{- Rückenschule}

Rückenschulungen basieren auf unterschiedlichen Konzepten. Hierbei sollte laut der „Nationalen Versorgungsleitlinie Kreuzschmerz" (Version 1.2., 2011) bei der Behandlung von (chronischen) Rückenschmerz-Patienten darauf geachtet werden, dass sich die Programme an einem biopsychosozialen Ansatz orientieren sowie an modernen Konzepten, wie z.B. Angst-Vermeidung („fear-avoidance“) („Nationale Versorgungsleitlinie Kreuzschmerz", Version 1.2., 2011, S. 28) und Wiederherstellung der Funktionsfähigkeit („functional restoration“) („Nationale Versorgungsleitlinie Kreuzschmerz", Version 1.2., 2011, S. 28). Die in Rückenschulen gelegentlich anzutreffenden Ratschläge i.S. von klassischen „Richtig-Falsch-Dichotomien“ („Nationale Versorgungsleitlinie Kreuzschmerz“, Version 1.2., 2011, S. 28) sind abzulehnen.

\section{- Thermotherapie}

Bei akutem nicht-spezifischen Kreuzschmerz ist eine Wärmebehandlung mit Wärmepflastern in der Anfangsphase möglich, da diese nicht bewegungseinschränkend für den Patienten sind. Jedoch muss auch hier wieder darauf geachtet werden, dass es zu keiner Unterstützung der Passivität des Patienten kommt, so dass sie nur in Kombination mit aktivierenden Maßnahmen Anwendung finden sollten.

Bei chronischem Kreuzschmerz sollen daher keine verschreibungspflichtigen Thermotherapien stattfinden. Gegen die Anwendung von Wärmflaschen und 
Körnerkissen ist nichts einzuwenden, solange sie nur als unterstützende Maßnahmen zur Verbesserung des persönlichen Wohlbefindens dienen.

\section{- Verhaltenstherapie}

Liegen psychosoziale Risikofaktoren vor (s.o., yellow flags), sollten beim Patienten mit akuten Kreuzschmerzen Techniken aus der kognitiven Verhaltenstherapie einbezogen werden. Bei chronischen Rückenschmerzpatienten soll dies im Rahmen multimodaler Behandlungsprogramme erfolgen.

\subsubsection{Medikamentöse Therapie}

Die medikamentöse Therapie soll symptom-orientiert erfolgen. Dabei soll sie den Patienten vor allem als Unterstützung dienen, damit Alltagsaktivitäten und therapeutische Aktivitäten, wie z.B. Krankengymnastik, möglich werden. Eine intensive Medikamentenanamnese vor Beginn der medikamentösen Therapie ist hierfür unerlässlich. Wichtig ist, dass die Medikamenteneinnahme einem festen Schema folgt. Dabei darf die Überwachung der Medikation nicht vernachlässigt werden. Die Notwendigkeit der Analgetikagabe soll in regelmäßigen Abständen geprüft und ggf. neu evaluiert werden.

\section{- Nicht-Opioid-Analgetika}

\section{Paracetamol:}

Bei akutem nicht-spezifischen Kreuzschmerz von leichter bis mittlerer Intensität kann Paracetamol mit einer Tageshöchstdosis von $3 g$ gegeben werden.

Bei subakutem und chronischem nicht-spezifischen Kreuzschmerz ist eine längere Einnahmedauer von Paracetamol nicht empfohlen. Schmerzepisoden, die kurzfristig mit verstärkter Intensität auftreten, können jedoch für kurze Zeit und niedrig dosiert mit Paracetamol behandelt werden. 
Immer bedacht werden sollten die möglichen unerwünschten Arzneimittelnebenwirkungen, die dieses Medikament hervorrufen kann. So kann z.B. bei längerer Einnahme ein Hypertonus entstehen. In Kombination mit NSAR müssen mögliche gastrointestinale Beschwerden und Blutungen bedacht werden.

\section{NSAR:}

Sowohl ein akuter nicht-spezifischer Kreuzschmerz als auch ein chronischer nichtspezifischer Rückenschmerz können für kurze Zeit in geringer Dosierung mit NSAR oral behandelt werden. Die parenterale Gabe wird nicht empfohlen.

Insgesamt sollten die Tagesdosen von Ibuprofen mit 1,2 g, für Diclofenac mit 100mg und für Naproxen mit 750mg nicht überschritten werden. Unter Betrachtung der möglichen unerwünschten Arzneimittelwirkungen (gastrointestinale Beschwerden) kann Ibuprofen auf 2,4g, Diclofenac auf 150mg und Naproxen auf 1,25g Maximaldosis erhöht werden.

Bei Patienten, die zu Magen-Darm-Beschwerden neigen, sollte die prophylaktische Gabe eines Protonenpumpeninhibitors in Erwägung gezogen werden.

\section{COX 2-Hemmer:}

Cox 2-Hemmer können sowohl beim akuten als auch beim chronischen nichtspezifischem Kreuzschmerz bei NSAR-Unverträglichkeit als off label use verabreicht werden.

\section{- Opioid Analgetika}

Wird die Wirkung von Nicht-Opioid-Analgetika als ungenügend empfunden, können nicht-spezifische Kreuzschmerzen gemäß WHO-Stufenschema mit schwachen Opioiden behandelt werden. Hierzu zählen z.B. Tramadol und Tilidin/Naloxon. Opioide mit einem langsamen Wirkungsbeginn sind zu bevorzugen, um das Suchtpotential zu verringern. 
BTM-pflichtige Opioide sind ausschließlich von Schmerzspezialisten und innerhalb multimodaler Programme einzusetzen.

Wichtig ist die Einnahme der Opioide nach einem konkreten Zeitplan.

Wenn nach 4 Wochen bei akutem nicht-spezifischen Kreuzschmerz und nach 3 Monaten chronischem nicht-spezifischen Kreuzschmerz keine Verbesserung der Schmerzsituation unter Opioid-Anwendung eingetreten ist, sollten sie nicht weiter verwendet werden.

Akute oder subakute nicht-spezifische Kreuzschmerzen sollen nicht mit transdermalen Opioiden behandelt werden.

\section{- Muskelrelaxanzien}

Wenn Nicht-Opioid-Analgetika bzw. nicht-medikamentöse Maßnahmen keinen ausreichenden Behandlungserfolg gezeigt haben, können Muskelrelaxanzien, sowohl beim akuten als auch beim chronischen nicht-spezifischen Kreuzschmerz, angewendet werden. Jedoch müssen auch hier die möglichen unerwünschten Arzneimittelwirkungen, wie allergische Reaktionen, reversible Leberfunktionsschäden und Magen-Darm-Beschwerden, mit in Betracht gezogen werden. Außerdem sollte eine kontinuierliche Einnahme auf 2 Wochen begrenzt werden.

\section{- Psychopharmaka}

\section{Benzodiazepine:}

Aufgrund des hohen Suchtrisikos wird die Vermeidung von Benzodiazepinen (z.B. Tetrazepam) dringend empfohlen. Außerdem wird laut der „Nationalen Versorgungsleitlinie Kreuzschmerz" (Version 1.2., 2011) die Teilnahme an einem multimodalen Programm bei längerfristiger Benzodiazepin-Einnahme deutlich obstruiert. 


\section{Antidepressiva:}

Bei chronischen nicht-spezifischen Kreuzschmerzen können noradrenerge oder noradrenerg-serotonerge Antidepressiva als unterstützende Medikation verschrieben werden. Sie sollten jedoch nicht als Einzel-Therapeutikum verwendet werden.

Die Gabe von SSNRI-Antidepressiva wird hingegen nur bei deutlich ausgeprägter depressiver und ängstlicher Begleitsymptomatik empfohlen und sollte nicht kontinuierlich erfolgen.

\section{- Antiepileptische Medikamente}

Die Einnahme antiepileptischer Medikamente wie Gabapentin, Pregabalin und Carbamazepin wird nicht empfohlen.

\subsubsection{Invasive Verfahren}

Hierzu werden perkutane und operative Verfahren gezählt.

Laut der „Nationalen Versorgungsleitlinie Kreuzschmerz" (Version 1.2., 2011) sollen bei nicht-spezifischen Rückenschmerzen generell keine invasiven Behandlungsverfahren angewendet werden. Sowohl für den akuten als auch den chronischen nichtspezifischen Rückenschmerz liegen keine verlässlichen Studien bezüglich der Effektivität der Verfahren vor.

\subsubsection{Prävention}

\section{- Aktivität}

Grundsätzlich steht beim nicht-spezifischen Kreuzschmerz die Aktivierung der Patienten im Vordergrund. Dabei kommt es weniger auf die Intensität der Bewegung an, viel bedeutsamer ist die Regelmäßigkeit der Aktivität. Hier ist für jeden Patienten individuell zu erarbeiten, welche Form der Aktivität für ihn am besten realisierbar ist. 


\section{- Edukation}

Einen wesentlichen Bestandteil der Behandlung von Rückenschmerzpatienten stellt die Wissensvermittlung und Informationsweitergabe betreffend des persönlichen Krankheitsbildes des Patienten dar, wobei der Betroffene Vorträge, Broschüren oder Bücher hinsichtlich Entstehung und Verlauf seiner Erkrankung erhält, um daraus ggf. Techniken zur Verhaltensänderung zu erlernen. Dieses Wissen soll wesentlich zum Gesundheitsverhalten beitragen. Ziel ist es, den Patienten auf Grundlage dieser Informationen zu selbstständigen körperlichen Betätigungen zu motivieren.

\subsubsection{Multimodale Therapie}

Laut der „Nationalen Versorgungsleitlinie Kreuzschmerz" (Version 1.2., 2011) sollen Patienten mit nicht-spezifischen Kreuzschmerzen so früh wie möglich in ein multimodales und interdisziplinäres Behandlungsprogramm einbezogen werden. Hierunter versteht man eine Therapieform, die mindestens 3 verschiedene Berufsgruppen involviert (Mediziner/Schmerztherapeuten, Psychologen/Psychotherapeuten, Physiotherapeuten, Ergotherapeuten) und in die nach einem gemeinsam abgestimmten Konzept spezifische ärztliche, psychotherapeutische und physiotherapeutische Maßnahmen einbezogen sind.

Besonders hervorzuheben ist, dass zumindest eine psychotherapeutische Fachrichtung gefordert ist (Arnold et al. 2009). Das zentrale Behandlungsziel ist die „Wiederherstellung der objektiven und subjektiven Funktionsfähigkeit mit Steigerung der Kontrollfähigkeit und des Kompetenzgefühls des Betroffenen“ (Arnold et al. 2009, S. 112-113). Bei Beschwerden, die trotz leitliniengerechter Therapie über 12 Wochen anhalten, sollte die Teilnahme an einem multimodalen Programm in Betracht gezogen und ggf. überprüft werden, beim Nachweis von sog. yellow flags (Risikofaktoren für die Chronifizierung, siehe Kapitel 2.4, S. 14) und gleichzeitigen persistierenden Beschwerden bereits nach 6 Wochen.

Arnold et al. (2009) nennen 2 Patientengruppen, für die multimodale Schmerztherapien besonders von Bedeutung sind: Patienten am Beginn eines Chronifizierungsprozesses, bei denen aufgrund ihrer rezidivierenden Beschwerden jedoch ein erhöhtes Risiko zur 
Chronifizierung besteht und Patienten mit bereits deutlich bestehendem Chronifizierungsstatus, deren ambulante monotherapeutische Behandlungen bisher nicht erfolgversprechend waren.

Weitere Indikationen für die Durchführung dieser Behandlungsform umfassen eine Änderung der Schmerzsymptomatik oder auch eine akute Schmerzdekompensation, einen zunehmenden Medikamentenverbrauch, das Vorliegen psychosozialer Risikofaktoren, die gesteigerte Inanspruchnahme des Versorgungssystems sowie eine zunehmende Gefährdung der Arbeits- und Erwerbsfähigkeit.

Klinisch länger anhaltende Wirksamkeitsnachweise sind laut Arnold et al. (2009) bisher jedoch nur für multimodale Programme mit einem Mindestumfang von 100 Behandlungsstunden bekannt.

\subsubsection{Versorgungskoordination}

In der Leitlinie sind in Abhängigkeit vom Stadium der Erkrankung Patientenwege beschrieben, in die die empfohlenen diagnostischen und therapeutischen Maßnahmen eingebettet sind (s. Abb. 3-5):

Bei einer über 2 Wochen andauernden Arbeitsunfähigkeit wird die Hinzuziehung weiterer Fachdisziplinen zur Meinung des Erstversorgenden empfohlen.

Bei erfolgloser Akutbehandlung über 2 bis 4 Wochen wird die Erhebung der yellow flags, der Risikofaktoren für eine Chronifizierung (siehe Kapitel 2.4, S. 14) empfohlen.

Bei Bestehen der Beschwerden von mehr als 4 Wochen trotz leitliniengerechter Therapie soll erneut eine psychosoziale Einschätzung erfolgen, sofern dies nicht bei der Eingangsdiagnostik berücksichtigt wurde. Bei bestehendem Verdacht auf eine psychosoziale Beteiligung ist bereits nach 2 Wochen andauerndem nicht-spezifischen Kreuzschmerz die Konsultation eines schmerztherapeutisch erfahrenen Psychologen oder Psychotherapeuten ratsam.

Bei einer Symptompersistenz von mehr als 6 Wochen sollen nochmals mögliche Chronifizierungs-Risikofaktoren überprüft werden.

Bestehen die Beschwerden länger als 12 Wochen, soll sich eine intensive somatische als auch psychosoziale Diagnostik anschließen. 
Diagnostik von „red flags“ und spezifischen Ursachen (0-2 Wochen nach Erstkonsultation)

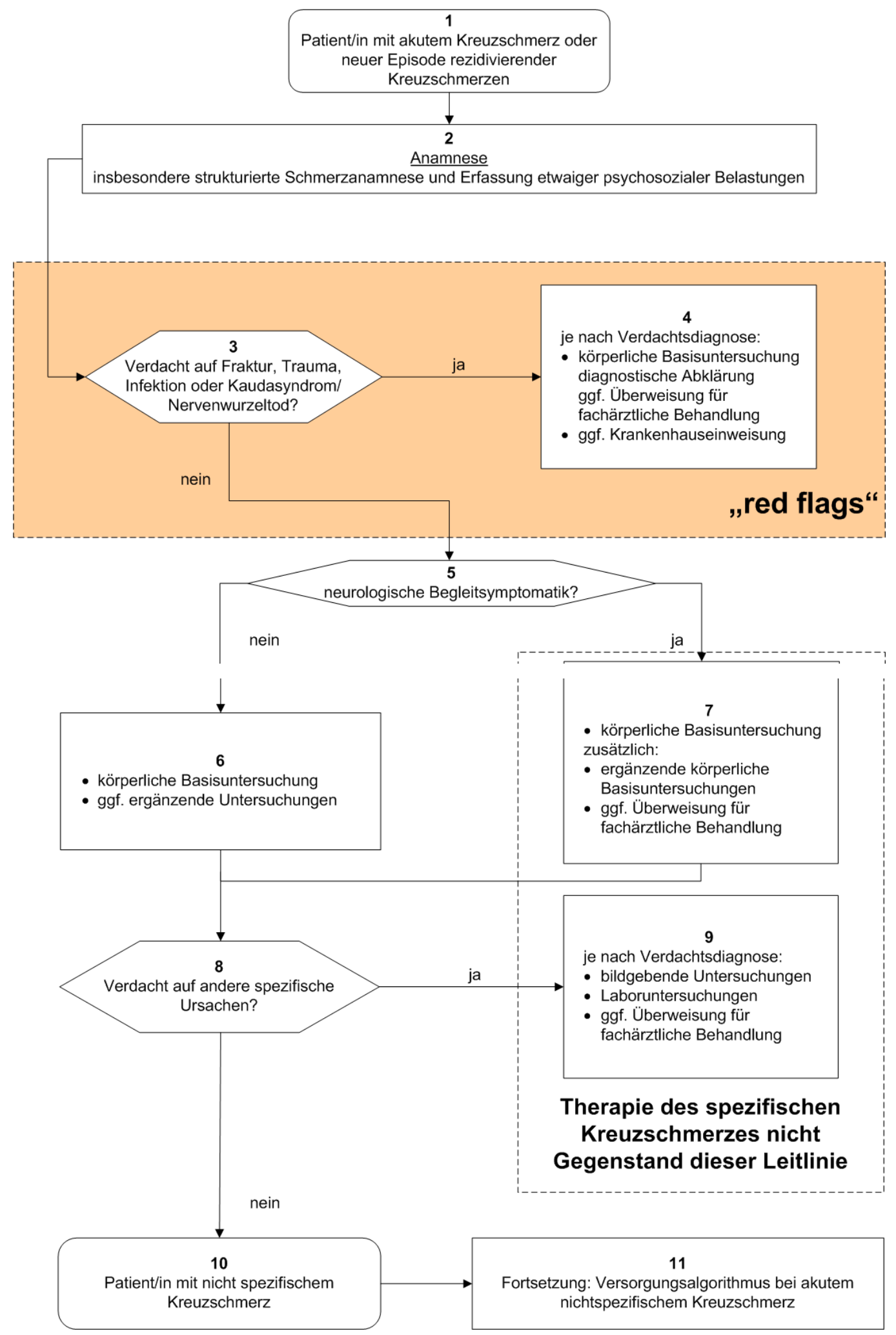

Abb. 3

Nationale Versorgungsleitlinie Kreuzschmerz - Kurzfassung. Version 1.2. (2011) 
Weitere Diagnostik und Therapie bei akutem nicht-spezifischem Kreuzschmerz (2-5 Wochen nach Erstkonsultation)

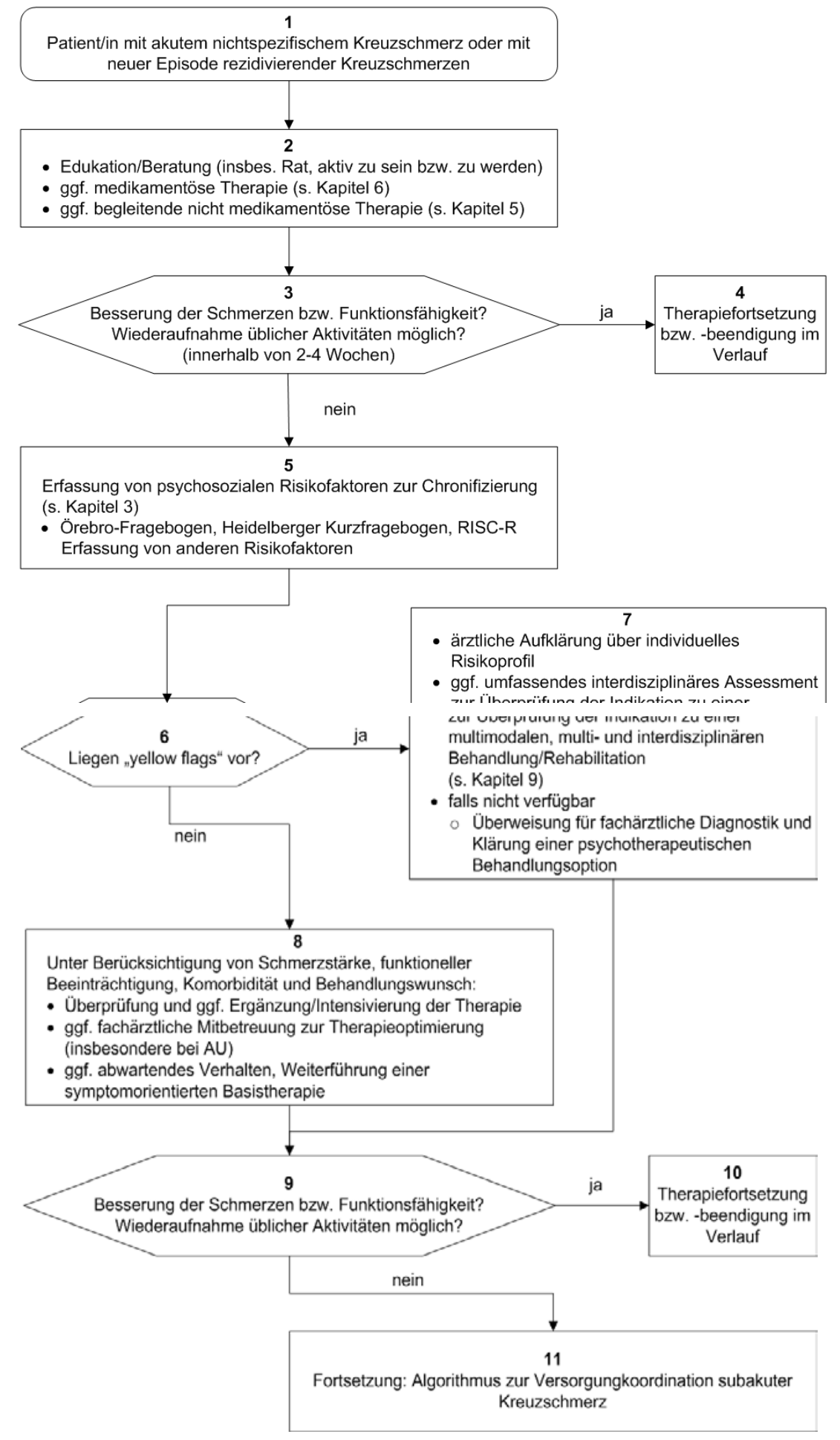

Abb. 4

Nationale Versorgungsleitlinie Kreuzschmerz - Kurzfassung. Version 1.2. (2011) 


\section{Versorgung bei subakutem Kreuzschmerz (6-12 Wochen nach Erstkonsultation)}

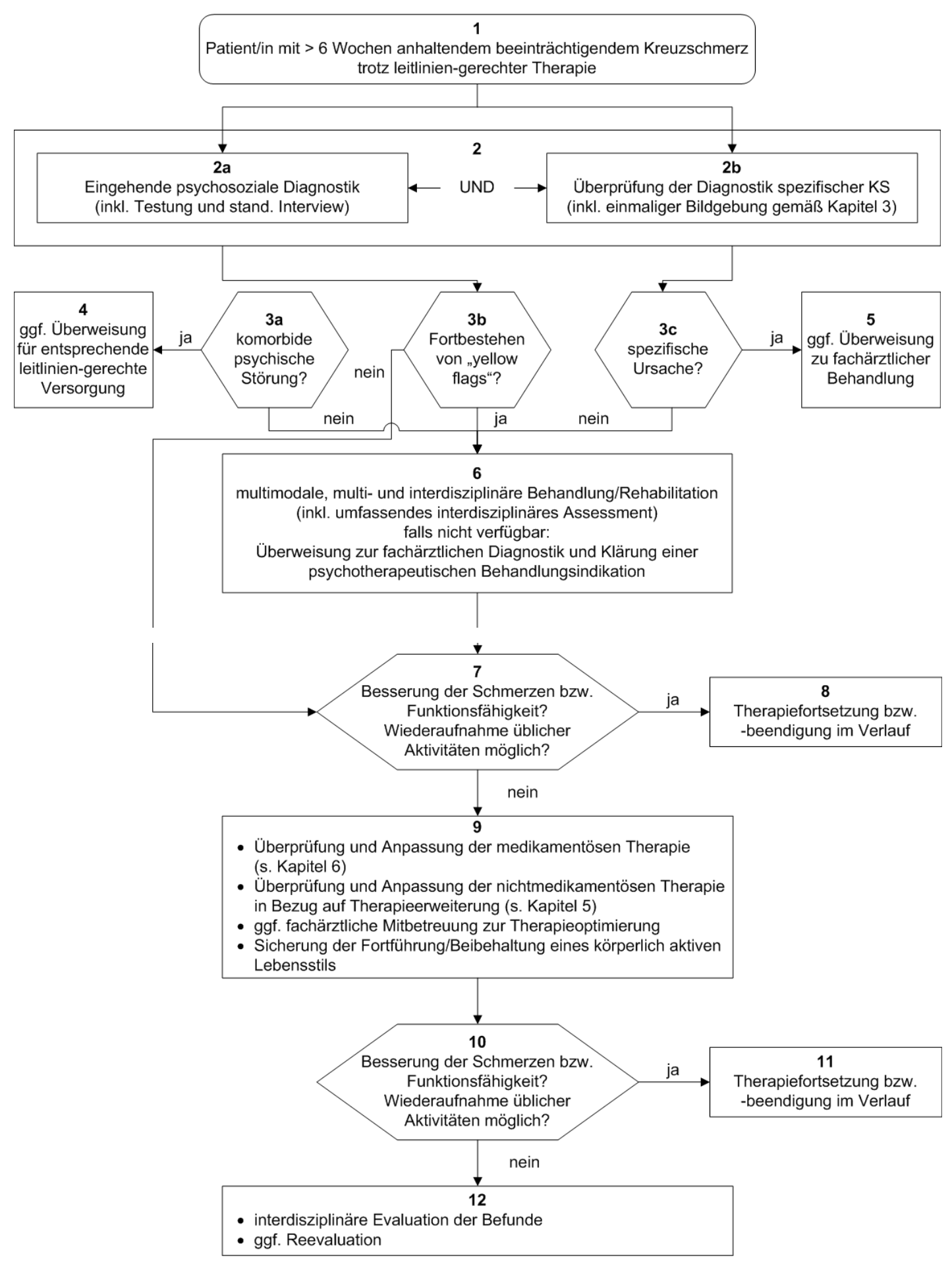

Abb. 5

Nationale Versorgungsleitlinie Kreuzschmerz - Kurzfassung. Version 1.2. (2011) 


\subsection{Bisherige Behandlung und Behandlungswege}

Da die "Weichen“ für den späteren chronischen Verlauf oft bereits am Beginn der Erkrankung gestellt werden, ist das Verhalten des Erstbehandlers von besonderer Wichtigkeit. Jüngste Studien haben diesbezüglich gezeigt, dass es deutliche Defizite in der Behandlungsvorgeschichte von Patienten mit Rückenschmerzen (Chenot et al. 2009 a) wie auch allgemein mit chronischen Schmerzen gibt (Schulte et al. 2009) und dass die Leitlinien-Adhärenz erheblich verbessert werden könnte (Feuerstein et al. 2006).

Eine größere Berliner Studie aus dem Jahr 2009 hat bereits einige wichtige Ergebnisse bezüglich der Patientenwege von Schmerzpatienten gezeigt (Schulte et al. 2009). Die Zielgruppe dieser Studie waren generell Schmerzpatienten, so dass neben Patienten mit Kreuzschmerzen (low back pain) auch Patienten mit Kopfschmerzen sowie neuropathischem Schmerz eingeschlossen wurden.

Die aus 3 Abschnitten bestehende Studie beschäftigte sich im ersten Kapitel mit Fragestellungen zum Thema Patientenwege. Es sollte geprüft werden, welche Arztgruppen bei chronischen Schmerzpatienten am häufigsten involviert sind, wer die Kontakte und mit welcher Häufigkeit initiiert und welche Zeitspannen zwischen den einzelnen Behandlungen liegen.

Im 2. Teil wurde das Behandlungsmanagement in verschiedenen Schmerzkliniken untersucht. Hier wurde unter anderem geprüft, ob die geforderte Interdisziplinarität (in multimodalen Programme) auch außerhalb von Forschungsstudien Eingang in normale Versorgung (Versorgungsrealität) gefunden hat. Auch der Ablauf der in den Schmerzkliniken durchgeführten Behandlungen (z.B. betreffend Kontaktintervalle, Medikationen etc.) wurde geprüft.

Der 3. Abschnitt beinhaltete die Überprüfung der Behandlungseffektivität 6 bzw. 12 Monate nach Beginn der Behandlung, woraus mögliche Prädiktoren für die Verbesserung des Schmerzerlebens und des Therapieerfolges abgeleitet werden sollten. 
Wie bereits erwähnt, standen im ersten Kapitel die im Zeitverlauf stattgefundenen Kontakte bis zum Beginn einer Behandlung in einem Schmerzzentrum im Mittelpunkt der Untersuchung.

Es galt herauszufinden:

- welches Intervall zwischen den ersten Schmerzsymptomen und dem ersten Arztbesuch im Durchschnitt liegt,

- wie die Besuchsfrequenzen, z.B. beim Allgemeinmediziner, sind,

- ob andere Fachrichtungen neben dem Hausarzt einbezogen werden, und wenn ja, welche,

- von wem die Überweisungen ausgingen.

- Des Weiteren wurden die diagnostischen Prozeduren und Resultate sowie die therapeutischen Interventionen vor dem Besuch einer speziellen Schmerzklinik untersucht, und

- die mittlere Zeitdauer ermittelt, bis ein Schmerzpatient in einer spezialisierten Schmerzklinik behandelt wurde.

Es wurden insgesamt 288 Patienten in die Untersuchung einbezogen: 136 Patienten mit Kopfschmerz (davon 79,6 \% weibliche Patienten), 99 mit Kreuzschmerzen (davon $69,7 \%$ weiblich) sowie 53 Patienten mit neuropathischem Schmerz (56,6\% weibliche Patienten).

Die Ergebnisse dieser Untersuchung zeigten, dass im Durchschnitt 12 Jahre zwischen dem Auftreten der ersten Symptome/ Beschwerden und der Vorstellung in einer spezialisierten Schmerzklinik vergingen. Weniger als die Hälfte der Rückenschmerzpatienten hatten zuerst ihren Hausarzt aufgesucht und zwar 1 bis 2 Monate, nachdem der Schmerz das 1. Mal aufgetreten war. Insgesamt war ein Allgemeinmediziner bei 88\% der Befragten mindestens einmal in den Krankheitsverlauf einbezogen. 55\% hatten jedoch schon direkt zu Beginn einen Spezialisten aufgesucht, zumeist einen Orthopäden. In 2/3 der Fälle wurde eine Überweisung an einen Spezialisten ausgestellt, entweder vom Allgemeinmediziner oder einem anderen Facharzt, am häufigsten für Neurologen, Orthopäden und Radiologen. Insgesamt waren 
pro Patient bis zur Vorstellung in einer speziellen Schmerzklinik durchschnittlich 3 Fachärzte in das Krankheitsgeschehen involviert, welches die teilweise problematische Primärversorgung widerspiegelt. Anstelle des vorrangigen Besuchs ihres Hausarztes entscheiden sich viele Patienten oft für den direkten Weg zu einem anderen Facharzt, der ihrer Meinung nach möglicherweise intensiver mit der Schmerzthematik vertraut ist.

Der 2. Abschnitt, der sich mit dem Schmerzmanagement in der speziellen Schmerzklinik beschäftigte, zeigte, dass nur in 32\% der Fälle eine interdisziplinäre Behandlung erfolgt war; ein Psychologe war z.B. nur bei ca. 1/3 der Patienten in die Behandlung einbezogen. Wie bereits im Kapitel 2.5.4 beschrieben ist jedoch bei der Behandlung von Schmerzpatienten (nicht nur mit chronisch fortgeschrittenem Krankheitsstatus, sondern vor allem auch mit einer beginnenden Chronifizierung) der Einbezug von psychotherapeutisch orientierten Ärzten unerlässlich (Arnold et al. 2009).

Im 3. Abschnitt der Studie zeigte sich letztendlich, dass lediglich $20 \%$ der erfassten Patienten eine klinisch relevante Verbesserung ihrer Schmerzen nach der Behandlung in den Schmerzzentren erreichten. Fast $1 / 3$ der Patienten nahmen bereits nach Erstvorstellung keine weiteren Folgetermine war. Nach 6 Monaten war nur noch die Hälfte aller befragten Patienten in der jeweiligen Schmerzklinik in Behandlung.

Die Autoren kommen aufgrund ihrer Ergebnisse zu dem Schluss, dass die Behandlung in einem Schmerzzentrum keine Garantie für eine Verbesserung der Schmerzsituation oder Lebensqualität von chronischen Schmerzpatienten gibt. Die Ergebnisse dieser Studie zeigten u.a., dass die Funktion des Allgemeinmediziners, dem eigentlich die Erstsichtung und Zuweisung des Patienten an die jeweiligen Institutionen obliegen sollte ("a constant attendant and coach of the pain") (Schulte et al. 2009, S. 6), nicht so wahrgenommen wird, wie es wünschenswert wäre. Für mehr als $50 \%$ aller Schmerzpatienten war der Allgemeinmediziner nicht die erste Kontaktperson.

Laut der Berliner Studie hatten die Patienten oft selbst ihre Zuweisung in eine Schmerzklinik initiiert. Dementsprechend ist es wenig verwunderlich, dass chronische Schmerzpatienten in vielen Fällen diese spezielle Hilfe sehr spät in Anspruch nehmen. Die Studie verweist außerdem auf Chenot et al., die bereits 2009 die hohe Frequenz, mit der in Deutschland ärztliche Spezialisten aufgesucht werden, beschrieben hatten. Laut Schulte et al. (2009) könnte dieses insofern nicht funktionierende „primary care- 
gate system" (Schulte et al. 2009, S. 6) eines der Gründe für die hohen Konsultationsraten von Fachärzten sein. Zugleich sprechen sie von einem unstrukturierten Überweisungssystem in Deutschland ("unstructured patient referral system within German health care" (Schulte et al. 2009, S. 7); "non-systematic pathway by which patients arrive in the SPC" (Schulte et al. 2009, S. 7). 


\section{Ableitung der Fragestellung/}

\section{Problematik der Umsetzung von Leitlinien in der alltäglichen Versorgung}

Ziel der Studie war, die Behandlungsvorgeschichte von Patienten mit chronischen Rückenschmerzen chronologisch bis zur Vorstellung in einer spezialisierten Schmerzklinik (Schmerztagesklinik und Ambulanz der Universitätsmedizin Göttingen) zu erfassen, zu analysieren und vor allem in Bezug auf ihre Leitlinien-Kompatibilität zu prüfen.

Zur Planung unserer Studie sichteten wir zuerst die bereits vorhandene Literatur betreffend die Behandlungswege von chronischen Schmerzpatienten. Während unserer Recherchen stellte sich heraus, dass noch viele offene Fragen und Unklarheiten bezüglich dieser Thematik bestehen. Weil die Berliner Studie (Schulte et al. 2009, s.o.) ein sehr gemischtes Klientel mit unterschiedlichen Diagnosen untersuchte, ist es besonders für die Gruppe der nicht-spezifischen chronischen Rückenschmerzpatienten bisher nicht klar, wie ihr Behandlungsverlauf sich vor der Einweisung/Behandlung in einer spezialisierten Schmerzklinik gestaltet. Auch die Frage, wann welcher Patient zum Facharzt überwiesen wird und aus welchen Gründen dies geschieht, war noch nicht gut untersucht und somit ein Ziel unserer Untersuchung.

Unsere Studie sollte sich weniger auf einen Vorher-Nachher-Vergleich von schmerzrelevanten und/oder psychometrischen Daten (NRS, SES, PDI, SF-12, HADS) beziehen. Vielmehr wollten wir diese Daten dafür nutzen, um zu prüfen, an welchem Punkt ihrer Erkrankung sich unsere Patienten zum Zeitpunkt des erstmaligen Kontakts mit unserer Schmerzklinik befanden.

Ein erhebliches Problem ist die Tatsache, dass der Versorgungsalltag oftmals nicht mit dem aktuellen Fachwissen Schritt halten kann.

Grol \& Grimshaw (2003) berichten darüber, dass z.B. Internisten 20 wissenschaftliche Veröffentlichungen pro Tag lesen müssten, um ihr Wissen in Entsprechung zum aktuellen Forschungsstand zu halten (S. 1225); viele Ärzte könnten mit den rasanten Fortschritten der Forschung nicht mithalten. Weiterhin geben sie an, dass laut amerikanischen und niederländischen Studien die Therapie von 30-40\% aller Patienten 
nicht dem aktuellen Stand der Forschung entspräche und dass 20-25\% entweder unnötige oder sogar schädigende Behandlungen erhielten (S. 1225).

Bereits im Jahr 2010 hatten Scott et al. berichtet, dass es Wissenlücken bzw. Problematiken bzgl. der Bewertung von red flags, der Relevanz diagnostischer Bildgebung und dem Einbezug von Patientenmotivation (Ermunterung zu kontinuierlicher Aktivität) gibt. Auch der Gebrauch bestimmter Medikamente (Muskelrelaxantien und Opioide) sei bisher nicht optimiert. Die Autoren berichteten über sog. "knowledge gaps" (S. 392) in Bezug auf den Gebrauch bestimmter Behandlungsmethoden wie z.B. von Physiotherapie, Akupunktur und TENS (S. 392). Scott et al. (2010) kritisieren dabei, dass viele Ärzte bzw. generell medizinisches Personal ihren eigenen Überzeugungen oftmals mehr Beachtung schenken als aktuellen Forschungsergebnissen und Guidelines ("Health care providers often rely on shared beliefs and personal opinion rather than research evidence to make treatment decisions.” S. 392). Auch Grol \& Grimshaw (2010) beschreiben: „Even if doctors are aware of the evidence and are willing to change, to alter well established patterns of care is difficult." (S.1225). Scott et al. (2010) berichten von einer "know-do-gap" - der Diskrepanz zwischen "was ist bekannt" und "wie wird es umgesetzt" (S.393). Sie sind davon überzeugt, dass diese Lücke auch speziell auf die Behandlung von Rückenschmerzpatienten zutrifft ("A know-do gap clearly exists among primary care practitioners with respect to the diagnosis and treatment of LBP." S. 399).

Die für unsere Untersuchung als Grundlage dienende Berliner Studie war eher strukturell und chronologisch orientiert (Wann wurde wer involviert?). Außerdem lag ein gewisser Schwerpunkt auf der Thematik, welchen Anteil die Allgemeinmedizin im Behandlungsverlauf von chronischen Schmerzpatienten hat (Fand eine Konsultation eines Allgemeinmediziner statt, wenn ja: wann, wie oft, regelmäßig?). Außerdem stand die Schmerzklinik als behandelnde Einrichtung im Mittelpunkt der Untersuchungen. Unser Schwerpunkt der Analyse sollte dagegen eher bei der Diagnostik liegen (Wie verlief der Erstkontakt? Welche bildgebenden Verfahren wurden wie oft und vor allem mit welchem Ergebnis durchgeführt?). Nicht nur die Anzahl der durchgeführten 
diagnostischen Untersuchungen, sondern auch die Konsequenzen, die sich daraus ergaben, sowie die Patientenzufriedenheit sollten erfasst werden. Außerdem wollten wir untersuchen, inwieweit Praxen Aufklärungsarbeit leisten bzw. wie weit die Patienten über ihr Krankheitsbild informiert sind.

Darüber hinaus wollten wir feststellen, wie multimodal die ambulanten Therapiemaßnahmen für (chronische) Rückenschmerzpatienten angelegt sind (z.B.: Werden neben Fachärzten auch Psychologen konsultiert? Nach welchem Zeitintervall erfolgt die Beantragung einer Kur oder Rehabilitationsmaßnahme?).

Im Vergleich zu Schulte et al. (2009) war eine ausschließlich retrospektive Studie geplant. Wir erhofften uns dadurch, die Behandlungsvorgeschichte bis zum Beginn der Konsultation in der Schmerzambulanz genauer analysieren und so die vorgelegten Ergebnisse der Berliner Studie noch präzisieren zu können.

Folgende Fragestellungen sollten genauer untersucht werden:

- Wie verläuft der erste Arztkontakt?

- Welche ärztliche Fachgruppe wird von den Patienten als erstes aufgesucht?

- Welche Diagnostik und Therapien werden angewandt?

- Werden psychosoziale Risikofaktoren frühzeitig nachgefragt/berücksichtigt?

- An wen (Fachärzte) werden die Patienten überwiesen?

- Ist die Vorgehensweise in Bezug auf diagnostische und therapeutische Maßnahmen leitlinienorientiert?

- Wie ist die Patientenzufriedenheit mit den jeweiligen Fachgruppen?

Durch die erhaltenen Informationen erhofften wir uns Hinweise auf Schwachstellen im Versorgungssystem sowie die Möglichkeit, durch die Kenntnis dieser Defizite eine Verbesserung und Beschleunigung der Abläufe zu bewirken und letztlich auch die finanziellen Mittel sinnvoller einsetzen zu können. 


\section{Material und Methoden}

\subsection{Datengenerierung}

Die Studie wurde durchgeführt in der Schmerztagesklinik und -Ambulanz des Zentrums Anästhesiologie, Rettungs- und Intensivmedizin, Universitätsmedizin Göttingen.

Die Datengrundlage der Studie bilden:

a) die Auswertung von Arztbriefen und Befundberichten von Patienten mit Rückenschmerzen, die i.d.R. bei der Anmeldung der Patienten vorlagen,

b) die Ergebnisse des Deutschen Schmerzfragebogens (DSF, Nagel et al. 2012), sowie

c) die Ergebnisse einer standardisierten Patientenbefragung zur Behandlungsvorgeschichte.

Ad a) Wenn sich Patienten zur Diagnostik/ Behandlung in der Schmerztagesklinik und Ambulanz der Universitätsmedizin Göttingen anmelden, werden sie im Voraus aufgefordert, zur adäquaten Beurteilung ihres Krankheitsbildes und des Verlaufes möglichst alle medizinischen Unterlagen der letzten Jahre der Anmeldung beizufügen. Diese Unterlagen werden zusammen mit dem ebenfalls ausgefüllten Deutschen Schmerzfragebogen (DSF, s.u.) in einer Teamsitzung gesichtet und bewertet. Dann wird darüber entschieden, welchen Ärzten/ Psychologen/ Physiotherapeuten die betreffenden Patienten beim Erstkontakt vorgestellt werden. Anhand der Informationen aus den Unterlagen wird auch entschieden, ob ggf. vorab weitere diagnostische Befunde erforderlich sind (z.B. neurologische Diagnostik bei chronischen Kopfschmerzen).

Ad b) Personen-bezogene Daten (soziodemographische Daten), eine ausführliche Schmerzbeschreibung (auch Chronifizierungsstatus) sowie der Krankheitsverlauf der betroffenen Patienten wurden aus den bei jedem Patienten vorhandenen Angaben im Deutschen Schmerzfragebogen (DSF) entnommen (s.S. 130). Dieser liegt zusammen mit den Arztberichten/Befunden (s.o.) bei der Anmeldung in der Schmerzklinik vor. Beim DSF handelt es sich um einen standardisierten Fragenkatalog, der sich zur Schmerz- 
erfassung bei Patienten mit länger bestehenden Schmerzerkrankungen eignet. $\mathrm{Er}$ wurde als gemeinsames Erhebungsinstrument von den beiden deutschen Schmerzgesellschaften (DGSS und DGS) herausgegeben. Er stell eine wichtige „Informationsbasis" für die ärztliche und psychologische Anamnese dar sowie eine „Datenbasis“ für spätere ergebnis-bezogene Untersuchungen (Ergebnisqualität, Katamnesen).

Der Basis-Fragebogen enthält die demographischen Informationen (Alter, Geschlecht, Körpergewicht, Körpergröße) und lässt anhand der Bestimmung von Körpergröße und Körpergewicht die Bestimmung des BMI zu. Weiterhin beinhaltet der Fragebogen eine detaillierte subjektive Beschreibung des Schmerzes (Lokalisation, Charakteristik, zeitlicher Verlauf, Intensität) und der erlebten Schmerzqualität (SBL).

Einige Ergebnisse des Fragebogens wie z.B. die Schmerzintensität werden anhand von Numerischen Ratingskalen ermittelt. Darin nehmen die betroffenen Patienten eine Einteilung ihrer Schmerzintensität von 0 (überhaupt kein Schmerz) bis 10 (stärkster vorstellbarer Schmerz) vor.

Außerdem erfolgt die Erfassung schmerzlindernder und -verstärkender Bedingungen sowie Screening-Tests auf depressive und ängstliche Störungen (Hospital Anxiety and Depression Score (HADS); ADS) und Störungen des Beeinträchtigungserlebens (PDI).

Folgende Fragebogen bzw. Beurteilungen sind im DSF enthalten und werden in dieser Studie als einzelne Parameter berücksichtigt

\section{- $\quad$ Schmerzqualitätsliste (SBL)}

Die SBL enthält 12 Adjektive (dumpf, drückend, pochend, klopfend, stechend, ziehend, heiß, brennend, elend, schauderhaft, scheußlich, furchtbar) zur näheren Beschreibung der sensorischen und affektiven Schmerzempfindung. Die Items sind vierfach abgestuft zu beantworten ( $0=$ trifft nicht $\mathrm{zu}, 3=$ trifft genau $\mathrm{zu}$ ).

Zu den affektiven Items zählen „elend“, „schauderhaft“, scheußlich“ und „furchtbar“ (Handbuch Deutscher Schmerzfragebogen Version 2012.1, Nagel et al., S. 13). Die Auswertung erfolgt nur in Bezug auf die affektive Schmerzbeschreibung, indem die Scores der betreffenen 4 Items addiert werden. Ab einem Cutoff-Wert von 8 wird von 
einer markanten affektiven Schmerzbeschreibung gesprochen, die einen Hinweis darauf gibt, dass der affektiv-emotionale Anteil des Schmerzerlebens dieses Patienten überproportional hoch ausgeprägt ist.

- Hospital Anxiety and Depression Score (HADS)

Der HADS spiegelt die allgemeine und seelische Verfassung des Patienten in Bezug auf depressive und ängstliche Symptome innerhalb der letzten 14 Tage wider (DSF).

Der HADS besteht aus den 2 Subskalen $A$ und $D$ ( $A=$ Ängstlichkeit, $D=$ Depressivität), die wiederum aus jeweils 7 als Selbstaussagen formulierten Items bestehen, für die es 4 Antwortmöglichkeiten gibt (0-3). Ausgewertet wird der HADS anhand einfacher Summenscores, für jede der beiden Skalen getrennt. Ab 11 Punkten wird eine klinisch relevante Angst- bzw. Depressionssymptomatik angenommen (Molzen 2007).

- Allgemeine Depressionsskala (ADS) (Hautzinger, Bailer 1993)

Anhand der Allgemeinen Depressionsskala beurteilt der Patient, bezogen auf die letzte Woche, ob und wenn ja wie oft bestimmte "depressive Affekte, körperliche Beschwerden, motorische Hemmungen und negative Denkmuster" (ADS-Handbuch Uni Freiburg 2012) inn beeinträchtigt haben. Dabei werden u.a. folgende depressive Merkmale erfragt: Einsamkeit, Traurigkeit, Angst, Schlafdefizite, Konzentrationsstörungen, Antriebslosigkeit, Demotivation, Selbstabwertung, Zweifel am Leben.

Für jede Frage bestehen 4 Antwortmöglichkeiten:

$0=$ selten oder überhaupt nicht (weniger als einen Tag)

$1=$ manchmal (ein bis zwei Tage lang)

$2=$ öfters (drei bis vier Tage lang)

3 = meistens, die ganze Zeit (fünf und mehr Tage lang)

Ein ADS-Summenwert $>23$ Punkte ist als kritisch $\mathrm{zu}$ betrachten und bedeutet möglicherweise eine ernsthafte depressive Störung. 
- Pain Disability Index (PDI) (Dillmann et al. 1994)

Der Pain Disability Index gibt an, inwieweit 7 verschiedene Lebensbereiche des Patienten durch die Schmerzen (zum momentanen Zeitpunkt der Befragung) beeinflusst werden.

Diese sind: familiäre und häusliche Verpflichtungen, Erholung, soziale Aktivitäten, Beruf, Sexualleben, Selbstversorgung sowie lebensnotwendige Tätigkeiten.

Die Beantwortung der Fragen erfolgt anhand einer numerischen Ratingskala von null bis 10 (null= keine Beeinträchtigung; 10= völlige Beeinträchtigung)

Dementsprechend liegt das Ergebnis zwischen null und 70.

Außerdem erfolgt anhand des Fragebogens eine Erfassung von diagnostischen und therapeutischen Maßnahmen im Krankheitsverlauf (Umfang der bisherigen Behandlung, Fachrichtungen der wegen Schmerzen aufgesuchten Ärzte, Medikamenten-Anamnese, vorherige schmerztherapeutische Behandlungsverfahren, etc. (s. Anhang). Bzgl. der soziodemographischen Daten werden im Fragebogen auch Informationen zur sozialen Situation (Schul-/ Berufsausbildung, derzeitige Arbeitssituation und Rentenstatus) erfasst (DSF, Nagel et al. 2012). Auch ein möglicher Grad der Behinderung bzw. Mögliche vorliegende Minderung der Erwerbsfähigkeit werden thematisiert.

- Grad der Behinderung (GdB)

Laut dem Bundesministerium der Justiz bezieht sich der GdB auf Gesundheitsstörungen jeder Art (die eine „Regelwidrigkeit gegenüber dem für das Alter typischen Zustand" bedeuten) unabhängig von ihrer Ursache und beinhaltet jegliche Folgen der dadurch resultierenden Einschränkungen in allen Lebensbereichen, nicht nur auf das allgemeine Erwerbsleben bezogen. Der Grad der Behinderung ist ein „Maß für die körperlichen, geistigen, seelischen und sozialen Auswirkungen“ der aus der Gesundheitsstörung resultierenden Funktionsbeeinträchtigung (www.gesetze-iminternet.de, Stand Mai 2013).

- $\quad$ Minderung der Erwerbsfähigkeit (MdE)

Die Minderung der Erwerbsfähigkeit ist für die Beurteilung des Bezugs von Leistungen aus der gesetzlichen Unfallversicherung bedeutsam. Diese zahlt jedoch nur, wenn die 
Schädigung der betroffenen Person aufgrund eines Arbeits-/ Wegeunfalls bzw. durch eine anerkannte Berufskrankheit verursacht wurde. Kommt es dementsprechend zu solch einer Schädigung, so wird geprüft, ob dadurch eine Minderung der Erwerbsfähigkeit entstanden ist. Bei der Einordnung und Überprüfung orientiert man sich ausschließlich an Beeinträchtigungen, die das Berufsleben/ die Erwerbsfähigkeit beeinträchtigen. Dabei wird jedoch nicht die derzeitige berufliche Tätigkeit herangezogen, sondern die ganz allgemeine Erwerbsfähigkeit auf dem gesamten Arbeitsmarkt (It.„Wegweiser Berufsunfähigkeitsversicherungen“ 2012).

\section{- $\quad$ V.Korff-Graduierung}

Ein wichtiges Merkmal zur Unterscheidung des breiten Spektrums der unterschiedlichen Erscheinungsformen des Rückenschmerzes ist der Schweregrad gemessen an den Kriterien der Schmerzstärke und Funktionsbeeinträchtigung. International bewährt hat sich diesbezüglich die als "Graded Chronic Pain Status (GCPS)“ (Kohlmann, Schmidt 2005, S. 2) bezeichnete Graduierung von Michael von Korff.

Grundlage des GCPS-Fragebogens (von Korff-Graduierung) sind insgesamt sechs 10fach gestufte Numerische Rating-Skalen (von 0-10; $0=$ kein Schmerz, $10=$ stärkster vorstellbarer Schmerz), anhand derer die Einstufung der Schmerzstärke (aktuelle Schmerzstärke, maximale Schmerzstärke, durchschnittliche Schmerzstärke) sowie die Einordnung des Beeinträchtigungserlebens von bestimmten Aktivitäten (alltägliche Aktivitäten, Freizeitaktivitäten, berufliche Aktivitäten) erfolgt.

Die Auswertung geschieht auf Grundlage von Beeinträchtigungspunkten und der Schmerzintensität nach folgendem Schema:

Die charakteristische Schmerzintensität wird ermittelt, indem die aktuelle, maximale und durchschnittliche Schmerzstärke summiert, gemittelt und dann mit 10 multipliziert werden.

Die subjektive Beeinträchtigung wird dementsprechend anhand der Werte der schmerzbedingten Beeinträchtigung bei alltäglichen Aufgaben, Freizeit- und beruflichen Aktivitäten erhoben. Auch dieser Wert wird gemittelt und mit 10 multipliziert. 
Eine zusätzliche Frage gibt Auskunft über die Anzahl der Tage, an denen der Patient in den letzten Monaten schmerzbedingt nicht seinen gewohnten Tätigkeiten nachgehen konnte.

Der Wert der subjektiven Beeinträchtigung und die Anzahl der „Fehl“-Tage ergeben zusammen die Beeinträchtigungspunkte.

Anhand dieser Beeinträchtigungspunkte und der charakteristischen Schmerzintensität ist dann eine Einstufung in 4 Schweregrade möglich, die die Einteilung in dysfunktionale und funktionale chronische Schmerzen zulassen (Molzen 2007).

Ad c) Eine weitere Datengrundlage der Studie bildet eine standardisierte Befragung der einbezogenen Patienten. Inhalte der Befragung waren vor allem der bisherige Behandlungsverlauf bis zur Vorstellung in der Schmerzklinik aus Sicht der Patienten, die Erfassung der von den Ärzten durchgeführten diagnostischen und therapeutischen Maßnahmen sowie die Zufriedenheit der Patienten mit diesen Maßnahmen. Der Fragenkatalog wurde zunächst aus den Zielsetzungen der Studie heraus generiert, mit der Literatur abgeglichen (insbesondere Berliner Studie (Schulte et al. 2009)) und anschließend mit Prof. Dr. J. Chenot aus der Abteilung für Allgemeinmedizin abgesprochen, weil in dieser Abteilung eine gute Expertise bzgl. der Erfassung von Patientenvorbehandlungen besteht. Die auf diese Weise zusammengestellte standardisierte Befragung wurde anschließend in einer Pilotierung im September 2009 an 4 Patienten in Bezug auf Praktikabilität und Durchführbarkeit geprüft.

Dabei zeigte sich z.B., dass die Frage nach Behandlungsmaßnahmen noch um die Angabe der Initiatoren erweitert werden musste. Auch die Frage nach Rehabilitationsmaßnahmen wurde präzisiert (Reha oder Kur, Zeitraum, Ort, Erfolg). Neu aufgenommen wurde nach der Pilotierung die Frage nach möglichen Arztwechseln und deren Gründen, da sich gezeigt hatte, dass durchaus mehrere Ärzte derselben Fachrichtung teilweise sogar zeitgleich oder nacheinander im Behandlungsverlauf involviert waren.

Als schwierig deutete sich die Erfragung nach einer konkreten Anzahl der Behandlungen und Physiotherapie-Sitzungen an (bzw. der Anzahl von verordneten 
Rezepten). Manchen Patienten fiel es leichter, Zeiträume anzugeben, was widerum die Vergleichbarkeit der Patientenaussagen im Nachhinein erschwerte.

Nachträglich herausgenommen wurde die Erfragung des subjektiven Einflusses der Patienten auf den Behandlungsverlauf. Anhand einer Skala von 1 (das Gefühl nichts persönlich beeinflussen zu können) bis 5 (großer Entscheidungsspielraum von Seiten des Patienten) wollten wir erfragen, wie sehr die Patienten selbst das Gefühl haben, ihren Krankheitsverlauf mitbestimmen bzw. beeinflussen zu können. Diese Frage erwies sich jedoch als schlecht praktikabel, wurde nur schwer verstanden und erschien daher wenig valide. Außerdem verzichteten wir nach Auswertung der Pilotierungsergebnisse auf die Erfragung der Dauer der Erstuntersuchung. Auch diese Frage erschien uns aufgrund der teilweise beträchtlichen Erinnerungsverzerrungen nachträglich als wenig hilfreich.

Entsprechend der Ergebnisse aus der Pilotierung erfolgte die Erstellung einer adaptierten Fragebogen-Version, die in der Anlage ab S. 121 aufgeführt ist. Auf Grundlage der entsprechenden Vorerfahrungen, dem Ergebnis der Pilotierung sowie Ergebnissen der Literatur wurde zur Minimierung des recall-bias (Erinnerungsfehler) ein retrospektiver Befragungszeitraum von 3 Jahren berücksichtigt.

Die Durchführungszeit der Befragung lag im Mittel bei 45 Minuten pro Patient (30-90 Minuten). Bei der Befragung wurden keine Namen von vorbehandelnden Ärzten notiert, sondern lediglich das Fachgebiet.

Die Einstufung des Patienten bzgl. des Ausmaßes seiner Schmerz-Chronifizierung erfolgte regelhaft in der medizinischen Anamnese mit Hilfe des Mainzer Pain Staging System (MPSS), s. S. 129.

Anhand des MPSS lässt sich der Patient einem von drei Chronifizierungsstadien zuordnen. Die zugehörigen Items beziehen sich auf 4 verschiedene Bereiche: zeitliche Aspekte der Schmerzen (Auftretenshäufigkeit, Schmerzdauer, Intensität), räumliche Aspekte des Schmerzes (z.B. betroffene Körperregionen), das Medikamenteneinnahmeverhalten sowie die Inanspruchnahme des Gesundheitswesens aufgrund der Schmerzen (Gerbershagen et al. (o.J.)). 
Die Auswertung erfolgt durch Bildung von Achsensummen, die ebenfalls addiert werden. Anhand dieser Punkte erfolgt die Zuordnung in 3 Schweregrade, zunehmend mit Stadienziffer: 4-6 Punkte entsprechen Stadium I, 7-8 Punkte Stadium II und 9-12 Punkte einem Stadium III (Pfingsten et al. 2000).

\subsection{Studienablauf/ Patientenrekrutierung}

Ca. $50 \%$ des Klientels der Schmerztagesklinik und -ambulanz im Universitätsklinikum Göttingen entfallen auf Patienten mit bewegungsbezogenen Schmerzen, insbesondere Rückenschmerzen. Die Einrichtung weist eine Spezialisierung in diesem Bereich auf und hat seit Jahren eine entscheidende Stellung in der Konzeption und Durchführung sog. multimodaler Behandlungsprogramme.

In unsere Studie wurden in einem Zeitraum von Oktober 2009 bis September 2010 konsekutiv 50 Patienten mit chronischen Rückenschmerzen, die zur Behandlung in die Schmerztagesklinik und -ambulanz überwiesen worden waren, aufgenommen. Ihre Berücksichtigung war primär daran gebunden, ob nach der ausführlichen ärztlichen Untersuchung die Diagnose eines chronischen, nicht-spezifischen Rückenschmerzes gestellt worden war (s. Einleitung). Ein weiteres Kriterium für die Berücksichtigung in der Studie war ein hoher Schmerz-Chronifizierungsgrad im MPSS (s.o.). Wir wollten vorrangig solche Patienten aufnehmen, die bereits länger unter ihren Beschwerden litten, die in ihrer Alltagsfunktion relevant beeinträchtigt waren und die bereits umfangreiche Erfahrungen im medizinischen System gesammelt haben. Diese Patientengruppe lässt sich am besten von anderen Patienten differenzieren über die Ausprägung der Schmerzchronifizierung. Die Operationalisierung dieses Merkmales erfolgte anhand des oben erläuterten sog. Mainzer Pain Staging System. In unsere Studie eingeschlossen wurden Patienten mit einem MPSS Stadium Grad II und III. Diese Gruppe zeichnet sich aus durch a) starke und anhaltende Schmerzen, b) eine multilokuläre Ausbreitung der Schmerzen, c) eine hohe Medikamenteneinnahme (s.u.) und d) ein hohes Inanspruchnahmeverhalten. 
Von der Studie ausgeschlossen wurden Fibromyalgie-Patienten sowie Patienten mit multilokulären Schmerzsyndromen, multiplen Diagnosen und Rückenschmerzpatienten, die lediglich einem MPSS Grad I aufwiesen.

Wenn die Eingangskriterien erfüllt waren (chronischer nicht-spezifischer Rückenschmerz plus Schmerzchronifizierungsgrad II oder III), klärte der behandelnde Arzt der Schmerztagesklinik und -ambulanz die Patienten anhand einer schriftlichen Information (s. Anhang S. 118) über die Inhalte der Studie auf und erfragte die Bereitschaft zur Teilnahme. Die Patienten bestätigten eine Teilnahme und die entsprechenden Bedingungen dann mit ihrer Unterschrift (s. Anhang S. 119).

Vor der eigentlichen Befragung der Patienten verschaffte sich die Doktorandin (Teresa Helbing) einen ausführlichen Überblick über die jeweilige Patientenkarriere und den individuell verschiedenen Krankheitsverlauf anhand der vorliegenden Akten. Besonderer Wert wurde auf jegliche Fakten gelegt, die man bei nicht medizinischvorgebildeten Patienten schwierig erfragen könnte (z.B. spezielle radiologische Diagnosen, therapeutische Interventionen und Medikamente).

An einem der ersten 3 Behandlungstermine erfolgte dann die standardisierte Befragung. Für die Patienten entstand durch die Befragung kein zusätzlicher Termin in der Schmerzambulanz, da die Befragung direkt im Anschluss an einen der ersten drei Behandlungstermine durchgeführt wurde.

Nach stattgefundenem Patienteninterview erfolgte nochmals ein Abgleich der im Interview erhaltenen Informationen mit den vorliegenden Patientenbefunden und Akten. Unsicherheiten bezüglich sozialer Angaben (z.B. Rentenverfahren), Jahreszahlen, Facharzt-Richtungen und therapeutischen Behandlungsverfahren wurden in einem weiteren Gespräch (i.d.R. beim 4. Kontakt) geklärt und mögliche NichtÜbereinstimmungen zwischen Aktenlage und Patientenaussage korrigiert.

Unser Ziel war es, mindestens 50 Patienten auswerten zu können, so dass wir bei Erreichen von 56 Patienten die Befragung beendeten.

Keiner der 56 wegen Teilnahme ärztlicherseits angesprochenen Patienten hat die Teilnahme abgelehnt. Rückwirkend mussten jedoch Patienten, die die oben genannten 
Einschlusskriterien doch nicht erfüllten, von der Studie ausgeschlossen werden. So verzeichnete die Studie letztendlich eine Responserate von 89\% (50 von 56 Patienten).

\subsection{Auswertung}

Alle erfassten Daten wurden in Excel-Tabellen an gesicherter und für Externe unzugänglicher Stelle archiviert. Patientennamen wurden nur während des Befragungszeitraumes in der Excel-Tabelle registriert, nach dem Ende der Datenerfassung wurden die Namen der Patienten gelöscht und ab diesem Zeitpunkt waren die Daten anonymisiert.

Es handelt sich um eine weitgehend in deskriptiver Weise angelegte Studie. Eine statistische Berechnung von Gruppenunterschieden erfolgte allenfalls bzgl. Alter und Geschlecht sowie bzgl. schmerzbezogener Parameter (Schmerzintensität, MPSS). Für die statistische Auswertung wurde SPSS, Version 18 eingesetzt.

Sofern nicht gesondert erwähnt, ist bei der Ergebnisdarstellung von identischen Fallzahlen (50 Patienten) auszugehen. Sollte es sich um unvollständige Datensätze handeln, sind diese und die entsprechenden missing values kenntlich gemacht.

Aufgrund des geringen Stichprobenumfangs erfolgen prozentuale Angaben ohne Dezimalstelle.

An dieser Stelle muss betont werden, dass zwischen dem erstmaligen Arztkontakt unserer Patienten und unserer Befragung in manchen Fällen mehrere Jahre lagen, was die Validität dieser Ergebnisse deutlich einschränkt. Andererseits wurden in der Befragung nur dann Daten erhoben, wenn ein Patient sich über den Ablauf dieser ersten Untersuchung weitestgehend sicher war. Sobald Verunsicherungen im Erinnerungsvermögen auftraten, wurde die Befragung zu dem betreffenden inhaltlichen Punkt abgebrochen. 


\section{Ergebnisse}

Die in den Kapiteln 5.1 bis 5.5.4 dargestellten Ergebnisse wurden zum Zeitpunkt der erstmaligen Vorstellung der Patienten in unserer Schmerzambulanz erfasst.

\subsection{Patientenprofil}

Von 56 befragten Patienten wurden nach nochmaliger Überprüfung 50 Patienten in die Studie einbezogen. Allen teilnehmenden Patienten war zuvor ein nicht-spezifischer chronischer Rückenschmerz diagnostiziert worden. 6 Patienten wurden rückwirkend von der Studie ausgeschlossen, da wir uns nachträglich entschieden, jegliche Patienten mit Nebendiagnosen (wie Fibromyalgie) von der Studie auszuschließen und außerdem nur Patienten mit MPSS Grad 2 und 3 zu werten. Der Fokus unserer Studie sollte ausnahmslos auf nicht-spezifischen Rückenschmerzpatienten liegen.

Die Ergebnisse der Kapitel 5.1.1 bis 5.1.6 wurden dem Deutschen Schmerzfragebogen (DSF) entnommen (s. Kap. 4.1).

Die danach folgenden Ergebnisse (Kapitel 5.2 bis 5.5.3) wurden im Rahmen des Patienteninterviews ermittelt.

\subsubsection{Alter und BMI}

Bei den 50 befragten Patienten handelt es sich um 26 Männer und 24 Frauen, im Alter zwischen 23 und 63 Jahren; dies entspricht einem mittleren Alter von 48,5 Jahren (SD 7,63). 86\% der Befragten waren mindestens 40 Jahre alt. Das Durchschnittsalter der Männer (Spannweite 37 bis 59 Jahre) betrug 49 Jahre (SD 6,21). Das durchschnittliche Alter der Frauen (23 bis 63 Jahre) lag bei 48 Jahren (SD 9,18). Es bestand zwischen Männern und Frauen bzgl. der Altersverteilung kein statistischer Unterschied (T-Test: T $=0,38$; n.s.).

Die Männer waren größer und schwerer als die Frauen (Körpergröße 179,8cm vs. 165,7cm; Körpergewicht: $88,8 \mathrm{~kg}$ vs. $73,4 \mathrm{~kg}$ ); in beiden Variablen gab es einen signifikanten Unterschied (Körpergröße: $T=8,4 ; \quad p<0,000$; Körpergewicht: $T=3,9$, $\mathrm{p}<0,000$ ). Der durchschnittliche BMI-Wert betrug 28 (Spannweite 18 bis 40; SD 4,40). 
Im Vergleich Männer/ Frauen zeigte sich bzgl. des BMI-Wertes kein signifikanter Unterschied ( $T=0,42$; n.s.). Untergewichtig war einer der Patienten, 16 Patienten waren normalgewichtig (32\% der Befragten). 2/3 der Patienten (66\%) zeigten einen BMI von mindestens 25. Insgesamt waren unsere Befragten in 38\% der Fälle übergewichtig und in $28 \%$ der Fälle adipös (s. Abb. 6).

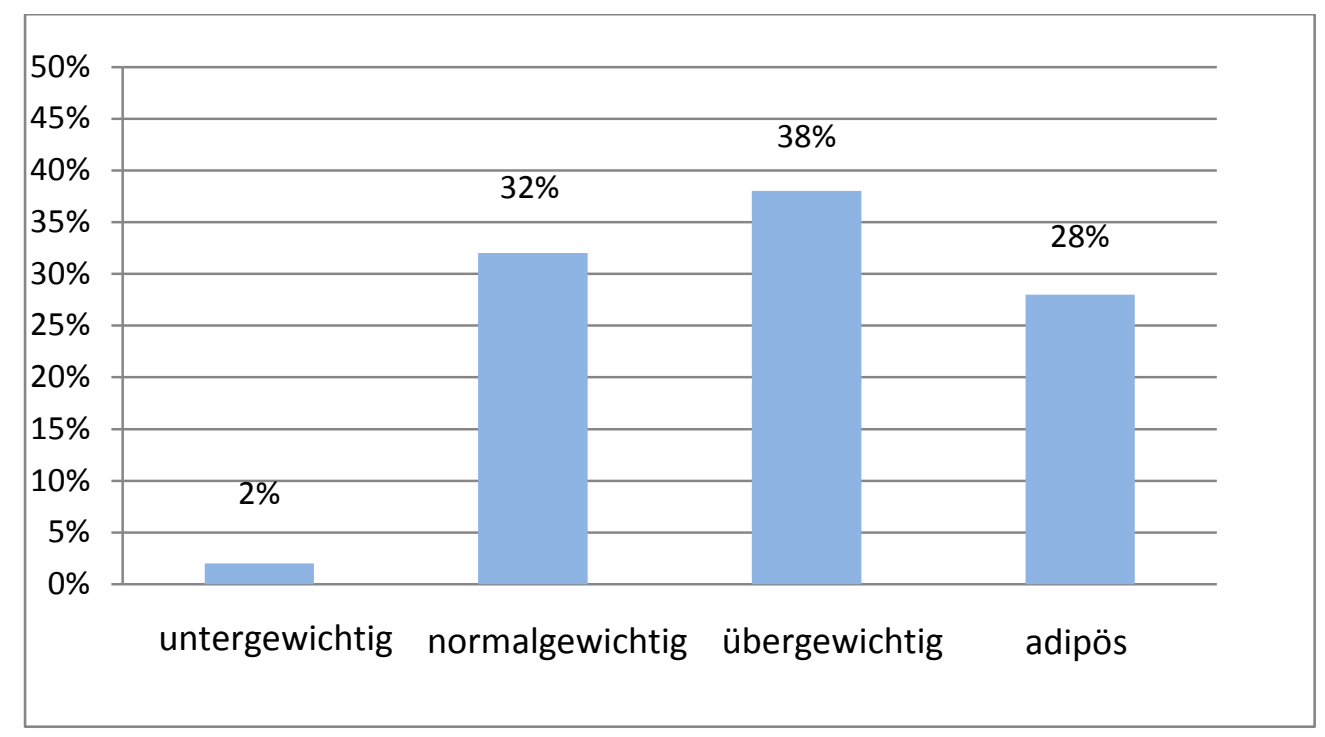

Abb. 6

BMI-Profil der befragten Patientengruppe $(n=50)$

\subsubsection{Soziale Angaben: Personenstand, Schulabschluss, berufliche Situation}

Der größte Anteil der befragten Personen war verheiratet (33 Patienten, 66\%). 16\% waren geschieden und 14\% ledig; 4\% der Befragten waren verwitwet. Von unseren Patienten verfügten $6 \%$ über keinen Schulabschluss. 34\% hatten einen Hauptschulabschluss, 50\% einen Realschulabschluss und 10\% Abitur.

Von den 50 Befragten waren n=12 (24\%) zum Zeitpunkt der Befragung nicht berufstätig. Gründe hierfür sind Studium ( $n=1)$, Arbeitslosigkeit $(n=3)$, Vorruhestand $(n=1)$ sowie befristete bzw. unbefristete Berentungen $(n=7)$ (siehe auch Kapitel 5.1.3). 
Von den 39 zum Zeitpunkt der Befragung berufstätigen Patienten befanden sich 49\% in einer Arbeiter-Position. 46\% waren in einem Angestellten-Verhältnis beschäftigt. Die verbleibenden $5 \%$ waren als Beamte oder Selbständige tätig.

\subsubsection{Laufende Rentenverfahren, MdE, GdB}

7 Patienten (14\%) waren zum Zeitpunkt der Befragung berentet.

Einen anerkannten Grad der Behinderung (GdB) wiesen 20 Patienten (40\%) auf. 80\% dieser Patienten hatten einen GdB von mindestens 30. Als Schwerbehinderung gilt ein GdB ab 50; diesen wiesen 5 der befragten Patienten auf (25\%)

Eine Minderung der Erwerbsfähigkeit aufgrund der Rückenproblematik lag bei $8 \%$ der Befragten vor.

\subsubsection{Rückenschmerzbezogene Krankschreibungen}

Bei den 39 berufstätigen Patienten (Ausschluss von nicht berufstätigen und von berenteten Patienten) lag die mittlere Dauer der Arbeitsunfähigkeit aufgrund der Schmerzen in den letzten 3 Jahren bei 9,1 Monaten; insgesamt schwankten die Angaben zwischen 1 und 20 Monaten (SD 6,94). 64\% (25 der 39 berufstätigen Patienten) beklagten mindestens 3 Monate Arbeitsausfall. Männer (19 gewertete Antworten) konnten dabei durchschnittlich 11,4 Monate (Spannweite 1 Woche bis 24 Monate; SD 6,91) aufgrund ihrer Schmerzen nicht am Berufsleben teilnehmen; Frauen (16 gewertete Antworten) fielen durchschnittlich 6,4 Monate aus (Spannweite 1 Woche bis 18 Monate, SD 6,15). Damit ergab sich ein signifikanter Geschlechtsunterschied bzgl. der Dauer der Arbeitsunfähigkeit $(T=2,4, p<0.05)$ in der Weise, dass Männer eine längere Arbeitsunfähigkeit aufwiesen.

\subsubsection{Schmerzintensität und Chronifizierung (MPSS)}

Auf der Numerischen Ratingsskala zur Erfassung der durchschnittlichen Schmerzintensität gaben die Befragten Werte von 4 bis 10 Punkten an. Dabei lag die 
durchschnittliche Schmerzintensität im Mittel bei 6,9 Punkten (SD 1,46). Bzgl. der durchschnittlichen Schmerzintensität ergab sich zwischen Männern und Frauen kein signifikanter Unterschied ( $\mathrm{T}=-1,2$, n.s.).

84\% der Befragten hatten eine durschnittliche Schmerzintensität von mehr als 5 NRSPunkten.

Die angegebene Schmerzdauer unserer Patientengruppe lag zwischen 1 und 30 Jahren. Davon gab mehr als die Hälfte der Befragten (56\%) an, zum Zeitpunkt der Befragung seit mindestens 10 Jahren Beschwerden zu haben.

Das mit dem Mainzer Pain Staging System (MPSS) ermittelte Chronifizierungsstadium unserer Patienten (nach dem ersten Behandlungstermin ermittelt) zeigte bei ca. 2/3 (68\%) ein Chronifizierungsstadium von Grad III; 16 Patienten (32\%) wiesen bzgl. des Chrofizierungsstadiums einen Grad II auf.

Bzgl. Schmerzintensität, Schmerzdauer und Chronifizierungststadium gab es keinen signifikanten Geschlechtsunterschied.

\subsubsection{Beeinträchtigungserleben und Depressions-Screening (PDI, ADS)} In Bezug auf das Depressions-Screening (s. Kap. 4.1) fanden wir bei unseren Patienten ADS-Werte zwischen 9 und 46 Punkten (s. Abb. 7). Der durchschnittliche ADS-Wert lag bei 26,5 Punkten (SD 10,03). 62\% der Befragten zeigten einen auffälligen Wert von über 23 Punkten. Davon waren 55\% weiblich. Vier der Befragten hatten sogar einen Wert von über 39 Punkten, was als hoch auffällig für eine depressive Symptomatik gewertet werden muss. Frauen erreichten im Mittel einen Wert von 27,3, während Männer im Mittel 25,7 aufwiesen; dieser Unterschied war statistisch jedoch nicht signifikant ( $T=-0,56$; n.s.). 


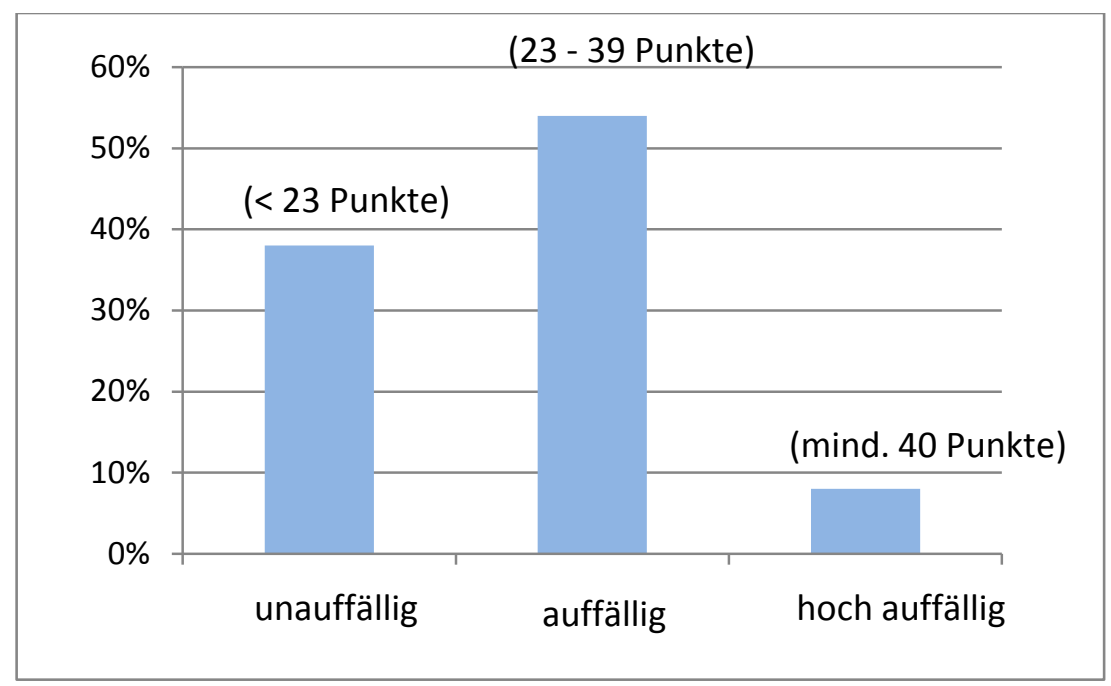

Abb. 7

Klassifizierte Werte der Allgemeinen Depressions-Skala (ADS)

Im Pain Disability Index (PDI) als Maß der erlebten Beeinträchtigung erreichten unsere Patienten Werte zwischen 16 und 67 Punkten. Daraus ergab sich ein Mittelwert von 37,6 Punkten (SD 12,44). Den grenzwertigen Summenwert (Cut-Off) von mehr als 44 Punkten überschritten 28\% unserer Patienten. Im Vergleich der Geschlechter erzielten Frauen mit 33,7 Punkten einen durchschnittlich niedrigeren Wert als Männer mit 41,2 Punkten (statisitsch signifikant, $\mathrm{T}=2,38 ; \mathrm{p}<0.05$ ).

Es zeigten sich korrelative Zusammenhänge zwischen der Schmerzintensität und dem Beeinträchtigungserleben im PDI $(r=.37, p<0.01)$ sowie zwischen der Schmerzintensität und dem Ausmaß der depressiven Symptome im ADS $(r=.44, p<0.01)$.

Die Ergebnisse der folgenden Kapitel (5.2 bis einschließlich 5.5.3) wurden anhand des Patienteninterviews ermittelt und dessen Inhalte mit den vorliegenden Arztbriefen und Befundberichten abgeglichen. 


\subsection{Erster Arztkontakt}

Nach dem erstmaligen Auftreten der Rückenbeschwerden suchten 24 Patienten (60\% bei 40 gewerteten Antworten) nach spätestens ca. einer Woche einen Arzt auf; 73\% innerhalb des ersten Monates. Diese Angaben basieren ausschließlich auf dem Erinnerungsvermögen der Patienten und können aufgrund der sehr komplexen und langjährigen Patientenkarrieren nicht sicher validiert werden.

In der Mehrzahl der befragten Fälle (63\%) stellte der Hausarzt den ersten Ansprechpartner dar (von 46 gewerteten Antworten). 24\% der Befragten suchten beim Erstkontakt direkt einen Orthopäden auf. Die verbleibenden 13\% suchten als erste Maßnahme Hilfe im Krankenhaus bzw. beim diensthabenden Bereitschaftsarzt.

\subsubsection{Diagnostische Erstmaßnahmen}

Bezogen auf die diagnostischen Erstmaßnahmen fragten wir nach der körperlichen Untersuchung, der Verschreibung/ Anfertigung bildgebender Diagnostik sowie der Berücksichtigung möglicher psychosozialer Risikofaktoren in der Anamnese/ Exploration.

\subsubsection{Körperliche Untersuchung}

Von allen Befragten (41 gewertete Antworten) gaben $95 \%$ an, beim Erstvorstellungstermin körperlich untersucht worden zu sein.

Um die Sorgfalt der körperlichen Untersuchung beurteilen zu können, wurde auch danach gefragt, ob die Patienten dafür ihre Kleidung ablegen mussten und ob sie sich an eine Palpation der Wirbelsäule erinnern. Das Ablegen der Kleidung zur Untersuchung geschah (laut den 40 gewerteten Patienten-Antworten) in 83\% der Fälle. Allerdings muss einschränkend festgehalten werden, dass Patienten häufig angaben, sich maximal halb entkleidet zu haben (nur den Oberkörper bis zur Hüfte).

Eine Palpation der Wirbelsäule erfolgte nach Angaben der Befragten in $88 \%$ der Fälle (bei 40 gewerteten Antworten).

In 52\% der Fälle (bei 40 gewerteten Antworten) sei auch der Reflexstatus erhoben worden. 


\subsubsection{Bildgebende Diagnostik}

In ca. der Hälfte der Fälle (51\% bei 43 gewerteten Antworten) wurde vom Erstbehandler direkt beim ersten Arztkontakt ein bildgebendes Verfahren veranlasst, dabei finden sich die Röntgenaufnahmen mit $64 \%$ an erster Stelle. Weiterhin wurden ein CT in 14\% der Fälle und ein MRT bei 23\% der Befragten beim ersten Arztbesuch verordnet.

Von 32 Patienten, deren erster Arztkontakt mit ihrem Hausarzt stattfand, wurde 13 von ihnen (41\%) ein bildgebendes Verfahren verordnet.

Von den 12 Patienten, die direkt einen Orthopäden konsultierten, wurden 7 Patienten (58\%) beim ersten Arztkontakt einer Bildgebung zugeführt.

Insgesamt bekamen 48 unserer 50 befragten Patienten (96\%) in den letzten 3 Jahren mindestens eine bildgebende Diagnostik aufgrund ihrer Rückenproblematik. Davon sind $78 \%$ der 50 befragten Patienten in den letzten 3 Jahren aufgrund ihres Rückenleidens mindestens einmal geröntgt worden. Noch häufiger (mit 80\%) wird die Erstellung mindestens einer MRT-Aufnahme in den letzten 3 Jahren angegeben. 60\% der Befragten berichteten über mindestens ein durchgeführtes $\mathrm{CT}$.

Andere Verfahren, wie z.B. Myelographien, Szintigraphien oder 3D-Ausmessungen der Wirbelsäule stellen Ausnahmen in der Diagnostik der befragten Gruppe dar.

\subsection{Diagnosemitteilung und Besprechung der Befunde}

Offensichtlich werden radiologische Befunde relativ häufig thematisiert (bei $80 \%$ der befragten Patienten). Über die Qualität der Befundübermittlung konnte jedoch wenig berichtet werden. Ein Großteil der Patienten (70\%) berichtete darüber, innen sei mitgeteilt worden, dass bei innen in der Bildgebung eine deutliche oder sogar schwere Schädigung des Rückens erkennbar sei. Nur 10\% der Befragten erinnerten sich daran, dass innen ärztlicherseits mitgeteilt worden sei, alles sei weitgehend normal.

Bzgl. der ihnen nach der Bildgebung mitgeteilten Befunde/ Diagnosen gaben $56 \%$ der Befragten an, dass ein Bandscheibenvorfall identifiziert worden sei.

Als weitere häufige Diagnose wurden arthrotische Veränderungen der Wirbelsäule erinnert (bei $1 / 4$ der Patienten). Auch Ileosakralgelenk-Blockierungen und Spinalkanalstenosen fanden sich auf den vorderen Plätzen. 
44\% unserer Befragten gaben an, mindestens 2 verschiedene Rücken-Diagnosen erhalten zu haben (s. Tab. 2)

\begin{tabular}{|l|l|}
\hline Anzahl Diagnosen & Anzahl Patienten \\
\hline 1 Diagnose & 28 Patienten (56\%) \\
\hline 2 Diagnosen & 12 Patienten (24\%) \\
\hline 3 Diagnosen & 8 Patienten (16\%) \\
\hline 4 Diagnosen & 2 Patienten (4\%) \\
\hline
\end{tabular}

Tab. 2

Anzahl der radiologisch ermittelten Diagnosen pro Patient

\subsubsection{Berücksichtigung psychosozialer Risikofaktoren}

Wie im Kapitel 2.4 bereits beschrieben, sind bestimmte psychosoziale (Risiko-Faktoren für eine Beschleunigung der Chronifizierung von Rückenschmerzen bekannt (sogenannte yellow flags). Um Chronifizierung zu verhindern, ist es deshalb hilfreich, diese psychosozialen Komponenten so früh wie möglich zu erfassen und möglichst in die erste Anamnese einzubeziehen. Darunter fallen Fragen zur familiären Situation, zum Beruf und zur Arbeitsplatzzufriedenheit, aber auch der Umgang mit den Schmerzen (sog. Schmerzbewältigung).

40\% der Befragten erinnern sich daran, dass Fragen zum beruflichen Bereich gestellt wurden; die Familiensituation wurde in $21 \%$ der Fälle (9 von 42 Antworten) erfragt, an Fragen nach dem Umgang mit den Schmerzen kann sich keiner unserer Patienten erinnern.

Im Verlauf der Erkrankung, d.h. mit zunehmenden Behandlungskontakten, ist ein verstärktes ärztliches Interesse an der Erfragung der beruflichen Situation zu verzeichnen: 37 von 50 Patienten (74\%) erinnern sich daran, dass zu einem späteren Zeitpunkt ihrer Erkrankung Fragen nach dem beruflichen Umfeld gestellt worden seien. 
Die familiäre Situation sowie der Umgang mit den Schmerzen seien im Verlauf nicht weiter thematisiert worden.

Einschränkend teilen viele der Befragten (ca. 40\%) mit, dass aufgrund des langjährig bestehenden Kontaktes zum Hausarzt, der sie schon viele Jahre betreuen würde, auf dessen Seite sehr gute Kenntnisse der privaten Situation vorliegen würden, so dass eine Befragung zu diesen Punkten nicht notwendig sei.

Im Laufe der Erkrankung wurde 30\% unserer befragten Patienten ärztlicherseits eine Psychotherapie empfohlen. Diese Empfehlung ergab sich nach Erinnerung der Patienten oftmals erst im Laufe der Behandlung (genauere Angaben sind nicht möglich). Diese Empfehlung wurde zu einem sehr großen Teil auch durchgeführt (in $80 \%$ der betreffenden Fälle).

11 der 15 Patienten (73\%), denen eine Psychotherapie im Laufe ihrer Behandlung empfohlen worden war, wiesen (später) im Deutschen Schmerzfragebogen einen auffälligen ADS-Wert über 23 auf. Der durchschnittliche ADS-Wert dieser Gruppe betrug 30,2 Punkte (Spannweite 9-42, SD: 11,25).

Einen erhöhten PDI-Wert, der den Cut-Off Wert von 44 überschreitet, wiesen 6 dieser 15 Patienten auf (40\%). Der durchschnittliche PDI-Wert der Patienten mit empfohlener Psychotherapie lag bei 42,3 Punkten (Spannweite 22-57, SD: 9,99). Die Patienten, denen eine Psychotherapie empfohlen wurde, zeigten anhand der NRS eine mittlere durchschnittliche Schmerzintensität von 7,5 (Spannweite 5-10, SD: 1,36) (siehe auch Tab. 3) 


\begin{tabular}{|l|c|c|}
\hline & $\begin{array}{l}\text { Patienten mit empfohlener } \\
\text { Psychotherapie }(n=15)\end{array}$ & $\begin{array}{l}\text { Restliche } \\
\text { Befragtengruppe }(\mathrm{n}=35)\end{array}$ \\
\hline Durschnittlicher ADS-Wert & 30,2 & 24,1 \\
\hline Durchschnittlicher PDI-Wert & 42,3 & 35,6 \\
\hline $\begin{array}{l}\text { Mittlere durchschnittliche } \\
\text { Schmerzintensität }\end{array}$ & 7,5 & 6,7 \\
\hline
\end{tabular}

Tab. 3

Depressive Symptome, Beeinträchtigungserleben und subjektive Schmerzintensität bei Patienten mit der Empfehlung, eine Psychotherapie aufzunehmen im Vergleich zu Patienten ohne eine solche Empfehlung

\subsubsection{Therapeutische Maßnahmen des Erstbehandlers}

Bzgl. der durchgeführten therapeutischen Maßnahmen (bei 44 Patienten) wurden in $73 \%$ der Fälle als erstes Analgetika verordnet (s. Abb. 8). In 34\% der Fälle wurden beim ersten Kontakt Injektionen in den Rücken verabreicht. 14\% gaben an, beim Erstkontakt Krankengymnastik/ Physiotherapie verordnet bekommen zu haben.

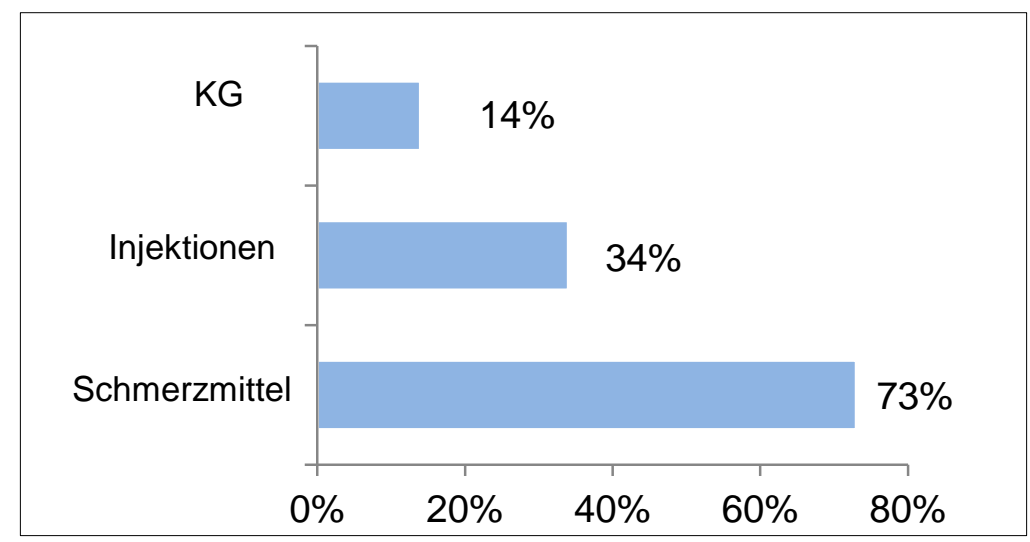

Abb. 8

Verordnete Maßnahmen im Erstkontakt

(Doppelnennungen möglich) 
Die am häufigsten von den Patienten genannten therapeutischen Maßnahmen (Schmerzmittel, Physiotherapie/ Sport, Rehabilitationsmaßnahmen, Operationen) haben wir genauer erfragt (s. Kap. 5.3.1- 5.3.4).

\subsection{Therapie}

\subsubsection{Medikamente (einschl. Aufklärung, Dosierung, Einnahmedauer, UAW)}

Die Medikamentenangaben wurden im Patienteninterview ermittelt und anhand des Chart Review und der Angaben im DSF überprüft und abgeglichen.

Die von uns befragten Patienten hatten aufgrund der Rückenschmerzen bis zum Zeitpunkt der Befragung durchschnittlich 3,9 verschreibungspflichtige Medikamente (Spannweite: 1 bis 8 Medikamente, SD: 1,94) im Verlauf ihrer Erkrankung eingenommen (s. Abb. 9).

Am häufigsten erinnern sich die befragten Patienten an die Verschreibung von Nichtsteroidalen Antirheumatika: Ibuprofen: 74\%, Diclofenac (Voltaren®): 46\%.

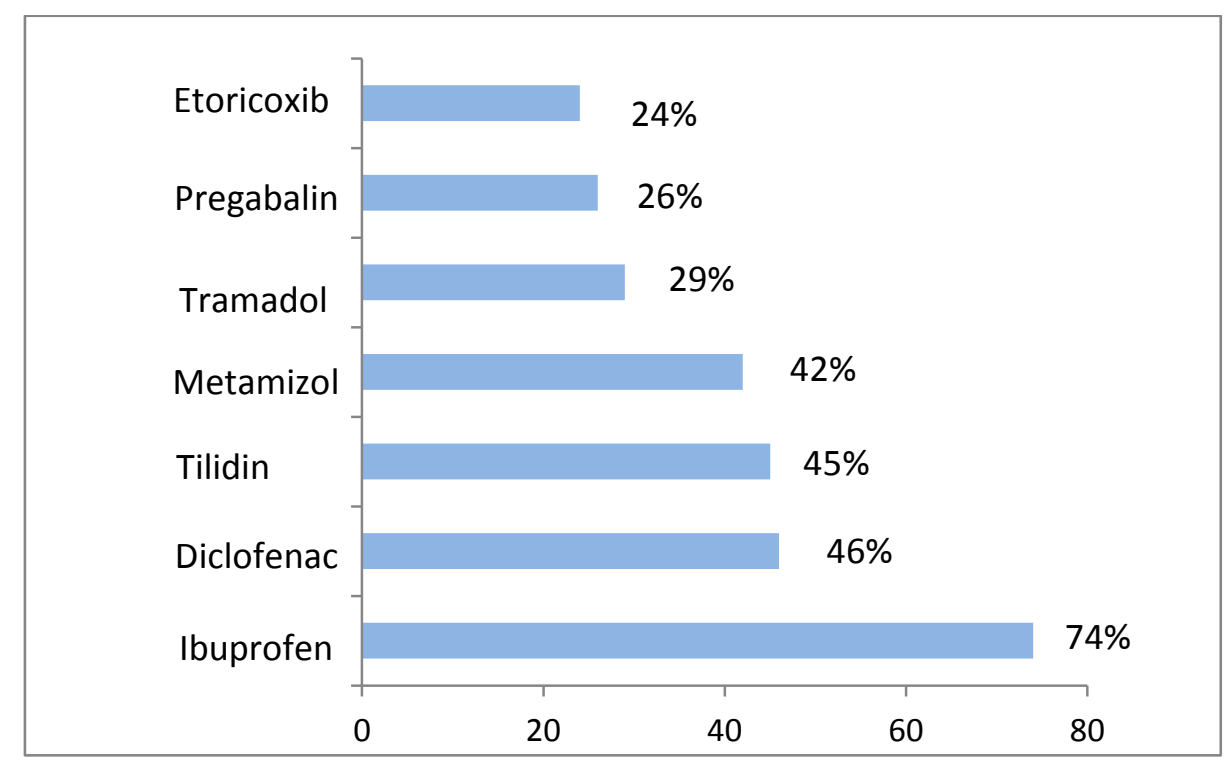

Abb. 9

Verschriebene Medikamente

(Doppelnennungen möglich) 
Weiterhin einen hohen Stellenwert besaßen Tilidin (Valoron ${ }^{\circledR}$ ), das 45\% der Befragte verschrieben bekamen, sowie Metamizol (Novalgin $\AA$ ). 42\% gaben an, dieses Medikament regelmäßig über einen längeren Zeitraum (mindestens 3 Monate) eingenommen zu haben. Mit geringem Abstand folgten Tramadol (29\%), Pregabalin (Lyrika $\left.{ }^{\circledR}\right)(26 \%)$ sowie Coxibe wie Etoricoxib (Arcoxia $\left.{ }^{\circledR}\right)$ und Celecoxib (Celebrex®) mit $24 \%$.

Auch trizyklische Antidepressiva wie Amitriptylin (16\%) und Benzodiazepine wie Tetrazepam (13\%) fanden mehrfach Anwendung. Eher gering vertreten waren Hydromorphon (Palladon $®)$ (9\%) und Gabapentin (7\%).

$20 \%$ der Befragten gaben an, zusätzlich freiverkäufliche Medikamente eingenommen zu haben; in $80 \%$ der Fälle war dies Paracetamol.

96\% der befragten Patienten gaben an, dass innen die Dosierung der Medikamente vom verordnenden Arzt erklärt worden sei (s. Abb. 10).

$28 \%$ seien zusätzlich explizit darauf hingewiesen worden, wie lange sie das gewählte Präparat einnehmen sollen. Sehr oft sei der Begriff bei Bedarf verwendet worden (72\%), Informationen über eine definierte und begrenzte Einnahmedauer und -häufigkeit habe es nicht gegeben.

$18 \%$ der Patienten gaben an, über etwaige unerwünschte Arzneimittelwirkungen aufgeklärt worden zu sein.

84\% der befragten Patienten berichteten darüber, Medikamenten-Nebenwirkungen erlitten zu haben. Diese beinhalteten zum größten Teil Übelkeit und Magenschmerzen (85\%) gefolgt von gastrointestinalen Beschwerden wie Diarrhoen bzw. Obstipation (35\%) Weitere mehrmals genannte unerwünschte Arzneimittelwirkungen waren Müdigkeit, Kopfschmerzen, Schwindel, Stimmungsschwankungen, Appetitlosigkeit sowie Wortfindungsstörungen und Schreibschwächen. 


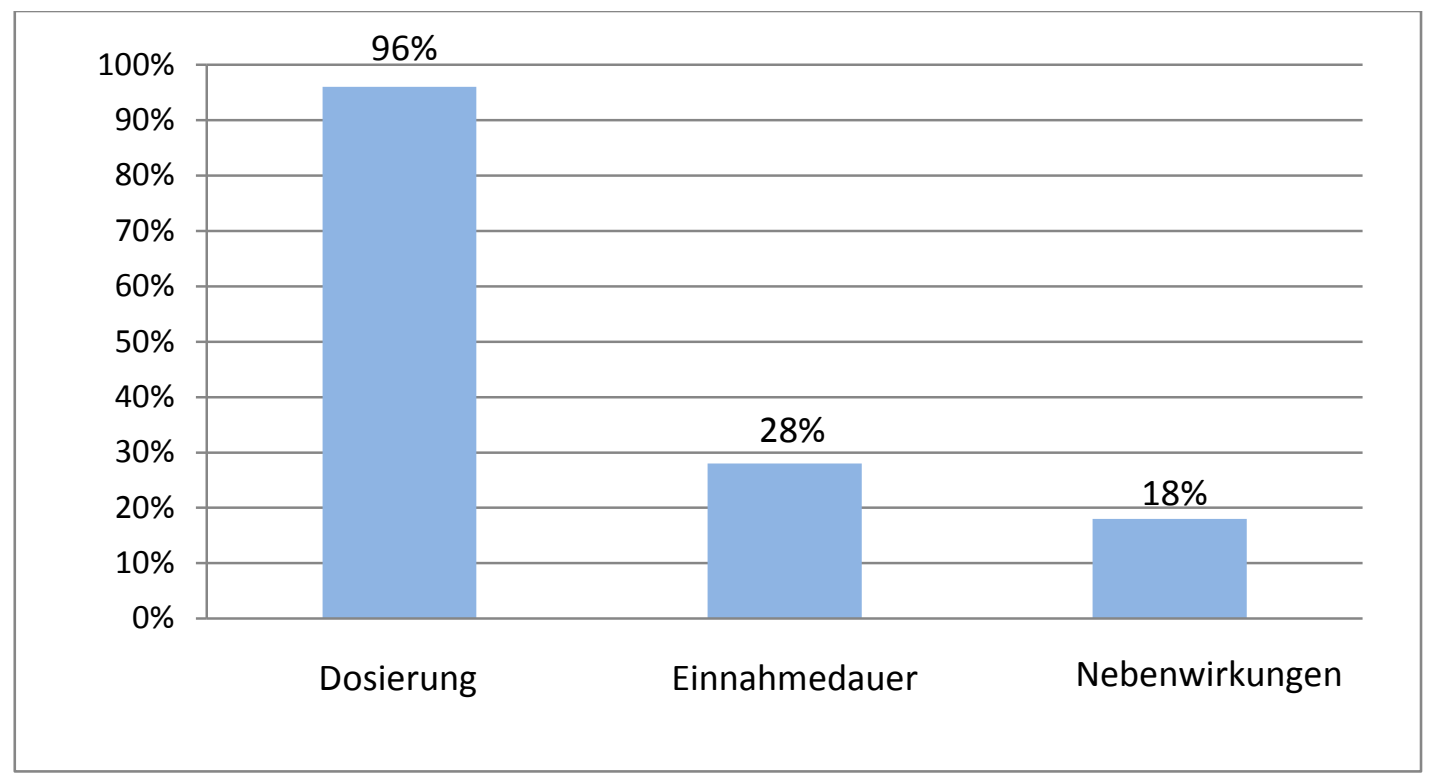

Abb. 10

Erinnerte Information zur Verordnung der Medikamente

\subsubsection{Physiotherapie}

Insgesamt wurden fast alle unserer Befragten (92\%) in den letzten 3 Jahren mit rückenspezifischer Physiotherapie behandelt. Davon erhielten unsere Patienten (basierend auf 41 Antworten) durchschnittlich 7,3 Rezepte à 6 Termine pro Person. (Spannweite 1-25 Rezepte, SD 6,06). Die Verordnung ging nahezu gleichermaßen sowohl von den Hausärzten (47\%) als auch von den Orthopäden aus (45\%). 8\% der Physiotherapie-Rezepte wurden von Neurochirurgen ausgestellt.

Die Frage, ob sie im Verlauf ihrer Behandlung von den Ärzten zu vermehrter körperlicher Aktivität motiviert worden seien, bejahten $76 \%$ der Befragten.

\subsubsection{Rehabilitationsmaßnahmen}

Insgesamt hatten 78\% unserer Studienteilnehmer bis zum Zeitpunkt der Befragung mindestens einmal während ihrer Erkrankung an einer Rehabilitationsmaßnahme teilgenommen. Bei 40\% der Befragten erfolgte noch ein 2. Rehabilitationsaufenthalt. 
Über einen dritten Aufenthalt berichtete noch fast $1 / 4$ der Patienten(24\%). In 10\% der Fälle erfolgte sogar eine 4. Rehabilitationsmaßnahme (s. Abb. 11).

Von den 39 Befragten, die von mindestens einer Rehabilitationsmaßnahme berichteten, blieben 39\% während ihres ersten Aufenthaltes für 3 Wochen in der Einrichtung; in 41\% der Fälle erstreckte sich der erste Aufenthalt über 4 Wochen.

44\% zeigten sich nach dem ersten Rehabilitationsaufenthalt sehr zufrieden und vergaben auf einer Skala von 1-5 (1: überhaupt nicht zufrieden; 5: sehr zufrieden) 4 bzw. 5 Punkte. Fast der gleiche Anteil aller Befragten (41\%) beurteilte den Erfolg ihrer ersten Rehabilitationsmaßnahme aber sehr kritisch und vergab nur 1 bzw. 2 Punkte.

Ca. 2/3 (65\%) aller, die mit der ersten Behandlung sehr zufrieden waren, nahmen auch an einer 2. Maßnahme teil. Von denen, die ihre erste Maßnahme als nicht erfolgreich eingeordnet hatten, beantragten fast die Hälfte (44\%) nochmals eine 2. Reha.

Die Patienten, die an 4 Rehabilitationsmaßnahmen teilnahmen, vergaben stets mindestens 3 Punkte auf der Zufriedenheitsskala.

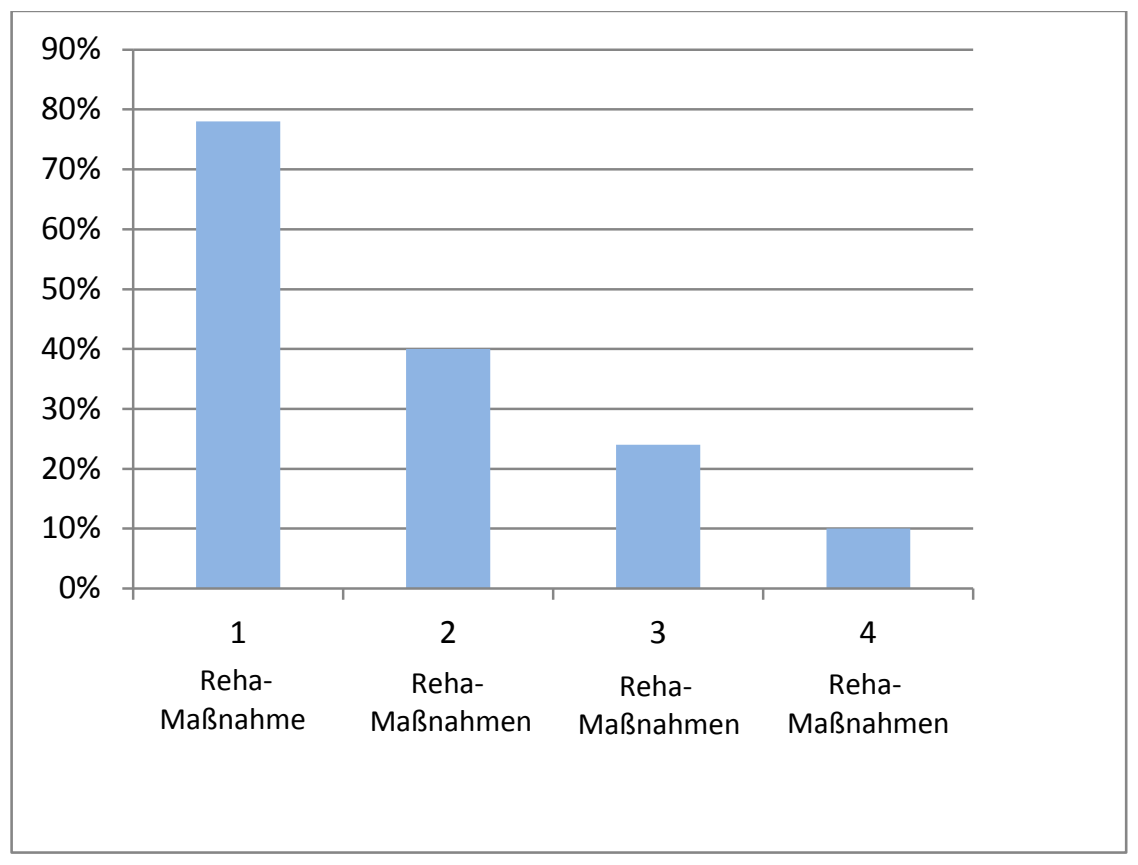

Abb. 11

Häufigkeit von Rehabilitationsaufenthalten 
Die Angaben zu den Rehabilitationsmaßnahmen wurden im Patienteninterview erfragt und anhand des DSF und des Chart Review auf ihre Richtigkeit und Vollständigkeit geprüft.

\subsubsection{Operationen}

Insgesamt $40 \%$ der befragten Patienten hatten seit Erstvorstellung mit Rückenschmerzen und bis zur Vorstellung in der Schmerzambulanz mindestens eine rückenbezogene Operation erhalten; $82 \%$ von ihnen bei einem Neurochirurgen oder in einer neurochirurgischen Abteilung. 18\% wurden von Orthopäden bzw. in einer orthopädischen Einrichtung operiert.

Die Angaben bezüglich stattgefundener Operationen wurden anhand des Chart Review überprüft.

\subsubsection{Weitere Behandlungen insgesamt}

Das Patienteninterview diente außerdem der Erfragung weiterer Behandlungsmaßnahmen, die zusätzlich zu Medikamenten, Physiotherapie, Rehabilitationsmaßnahmen und Operationen angewendet worden waren (s. Abb. 12). Bezogen auf den gesamten Behandlungsverlauf hatten $76 \%$ mindestens einmal Injektionen in den Rücken ohne eine begleitende Bildgebung erhalten. Injektionen in den Rücken mit Bildgebung sind bei knapp der Hälfte der Befragten 48\% erfolgt. An Genaueres (z.B. die spezielle Technik oder die verwendeten Medikamente) konnte sich fast niemand der Befragten erinnern.

Elektrische Behandlungsmethoden wie Reizstrom (68\%) oder die Benutzung eines TENS-Gerätes (57\%), sowie Manipulationen (57\%) (wie z.B. das „Einrenken“) folgen auf den Plätzen 2 und 3 der zusätzlich durchgeführten Maßnahmen. Mit Schuheinlagen oder einer kassenärztlich getragenen Rückenschulung wurden etwas über 1/3 der Patienten (jeweils 36\%) therapiert.

Die Rückenschulung (z.B. Gesundheitstraining im Göttinger Rehazentrum Rainer Junge $\mathrm{GmbH}$ ) sollte vor allem Personen mit Rücken- belastenden Berufen dienen sowie unspezifischen Rückenschmerz-Patienten den Umgang mit ihren Schmerzen 
erleichtern. Das Training beinhaltete Übungen zur verbesserten Körperwahrnehmung, Funktionsgymnastik, das Erlernen von Entspannungstechniken. Simuliert wurden außerdem alltägliche Bewegungen aus Beruf und Haushalt, anhand derer rückenschonende Bewegungen erlernt werden sollten. Muskuläre Dysbalancen sollten erkannt und Haltungsfehler behoben werden.

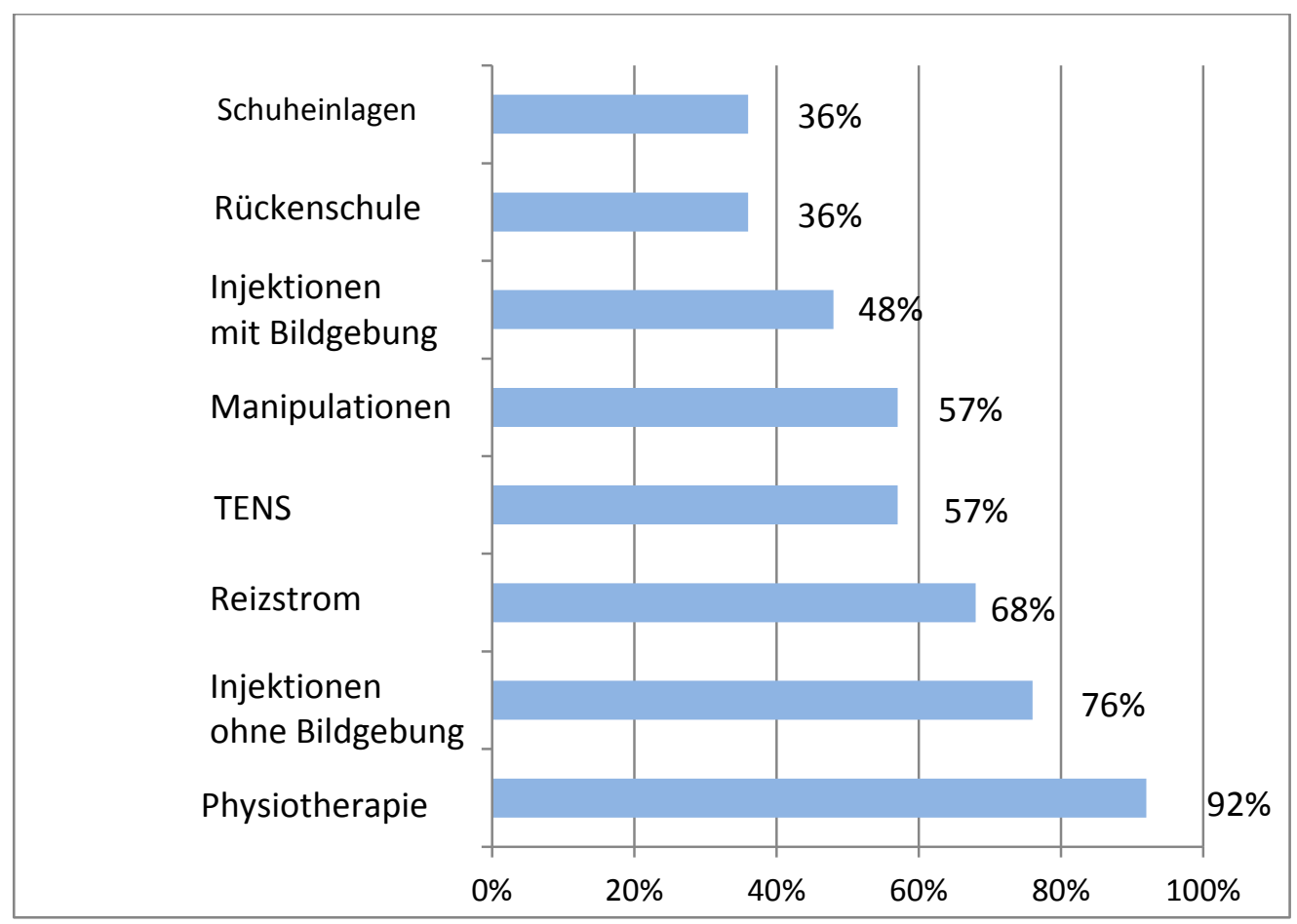

Abb. 12

Von den Befragten angegebene therapeutische Maßnahmen

\subsubsection{Selbst ergriffene Maßnahmen}

Rund 94\% aller Befragten gaben an, dass sie zusätzlich zu ärztlichen Interventionen selbstständig weitere Maßnahmen ergriffen haben, um ihre Beschwerden zu lindern. In fast 2/3 der Fälle (60\%) war dies der Kauf einer neuen Matratze (s. Abb. 13). 54\% kauften sich außerdem diverse Geräte bzw. Hilfsmittel (Heimtrainer, Hanteln, Terrabänder, Gymnastikbälle). 
Über die Teilnahme an verschiedenen Sportarten, wie regelmäßiges Schwimmen, Nordic Walking, Reiten und Wassergymnastik, wurde ca. von der Hälfte aller Patienten (49\%) berichtet.

42\% nahmen an Akupunktur-Sitzungen teil.

Entspannungs-Verfahren, wie Yoga, Pilates, Qi Gong, Tai-Chi und Feldenkrais wurden von $16 \%$ der befragten Patienten angegeben.

Fast $1 / 3$ der Patienten (32\%) investierte zusätzlich in Massagen, deren Kosten sie selbst trugen.

$16 \%$ suchten außerdem einen Osteopathen auf.

Bei der Erfragung der selbst ergriffenen Maßnahmen wurde intensiv auf die Trennung und Unterscheidung von Zuzahlungsleistungen und Selbstzahlerleistungen geachtet. Bezogen auf selbstbezahlte (Behandlungs- Maßnahmen zur Linderung der Rückenbeschwerden ergab sich bei Auswertung von 25 relativ differenzierten Antworten schätzungsweise ein Wert von um 2.250 Euro pro Patient (Spannweite 100 Euro bis 8.000 Euro; SD 2162,12).

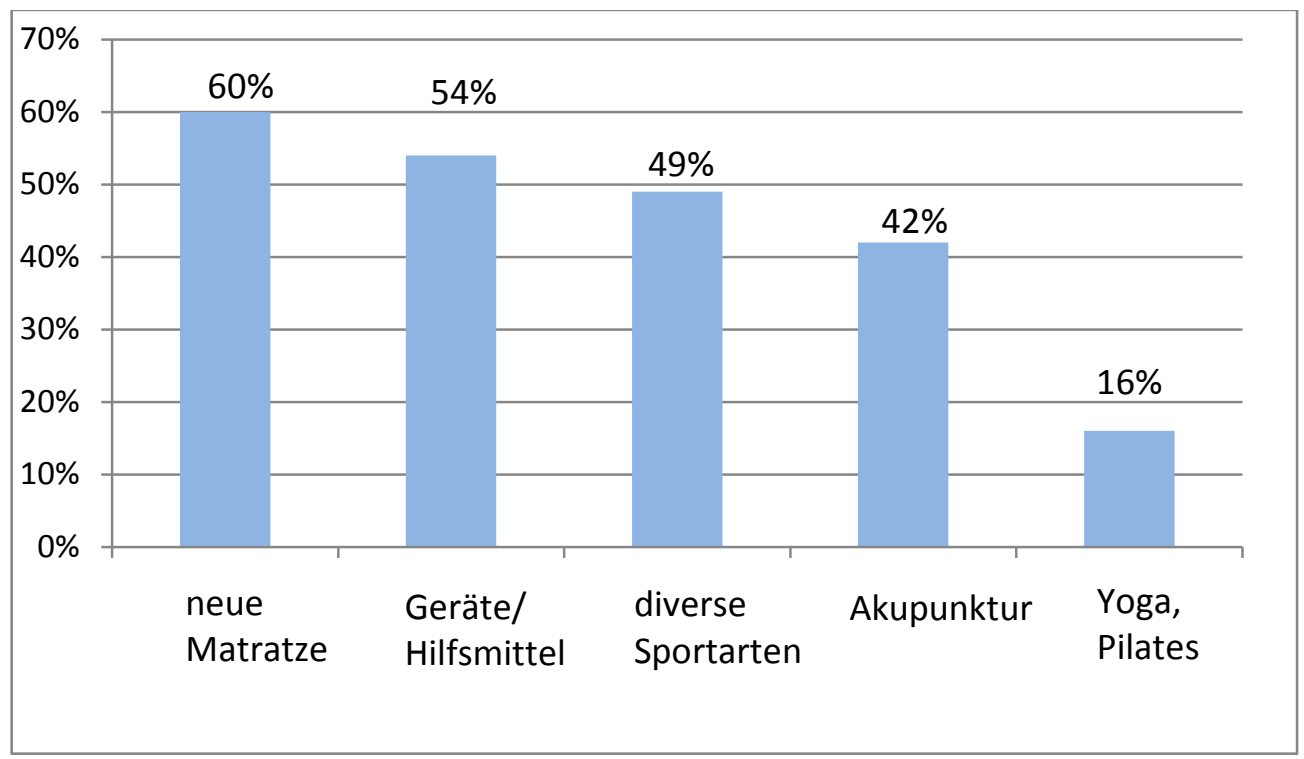

Abb. 13

Eigens angewendete Maßnahmen 


\subsection{Weiterbehandelnde Arztgruppen neben dem Hausarzt}

Im Patienteninterview erfragt und anhand des Chart Review nochmals überprüft erfolgte auch eine Erfassung bzgl. der weiterbehandelnden Arztgruppen.

\subsubsection{Fachrichtungen}

Wie schon im Kapitel 5.2.1 beschrieben, erfolgte bei unserer befragten Patientenstichprobe die Einbeziehung von Fachärzten zu einem relativ frühen Zeitpunkt. Überweisungen erfolgten an Orthopäden, Neurochirurgen, Neurologen, Psychologen, Rheumatologen und Algesiologen (s. Abb. 14).

In den meisten Fällen handelte es sich dabei um einen Orthopäden. 84\% aller befragten Patienten gaben an, bei mindestens einem Orthopäden langfristig in Behandlung (gewesen) zu sein. 52\% suchten wegen der Rückenbeschwerden einen Neurologen auf. $40 \%$ aller Befragten waren außerdem in neurochirurgischer Behandlung. Psychotherapeutische Betreuung erfolgte in $24 \%$ der Fälle. Fast $1 / 5$ der Patienten (14\%) hatte sich außerdem von einem Heilpraktiker behandeln lassen.

In diese Statistik nicht einbezogen wurden die Radiologen, da diese zum größten Teil ausschließlich für die Befundung zuständig waren und kaum einen Einfluss auf die Behandlung hatten.

Wichtig festzuhalten ist, dass viele Patienten oft mehrere Ärzte derselben Fachrichtung (zum Teil drei verschiedene Orthopäden gleichzeitig) parallel besuchten.

Insgesamt ergab sich inklusive Hausarzt eine durchschnittliche Behandler-Anzahl von 3,3 Ärzten (Spannweite 1-5 Ärzte; SD 1,02). 


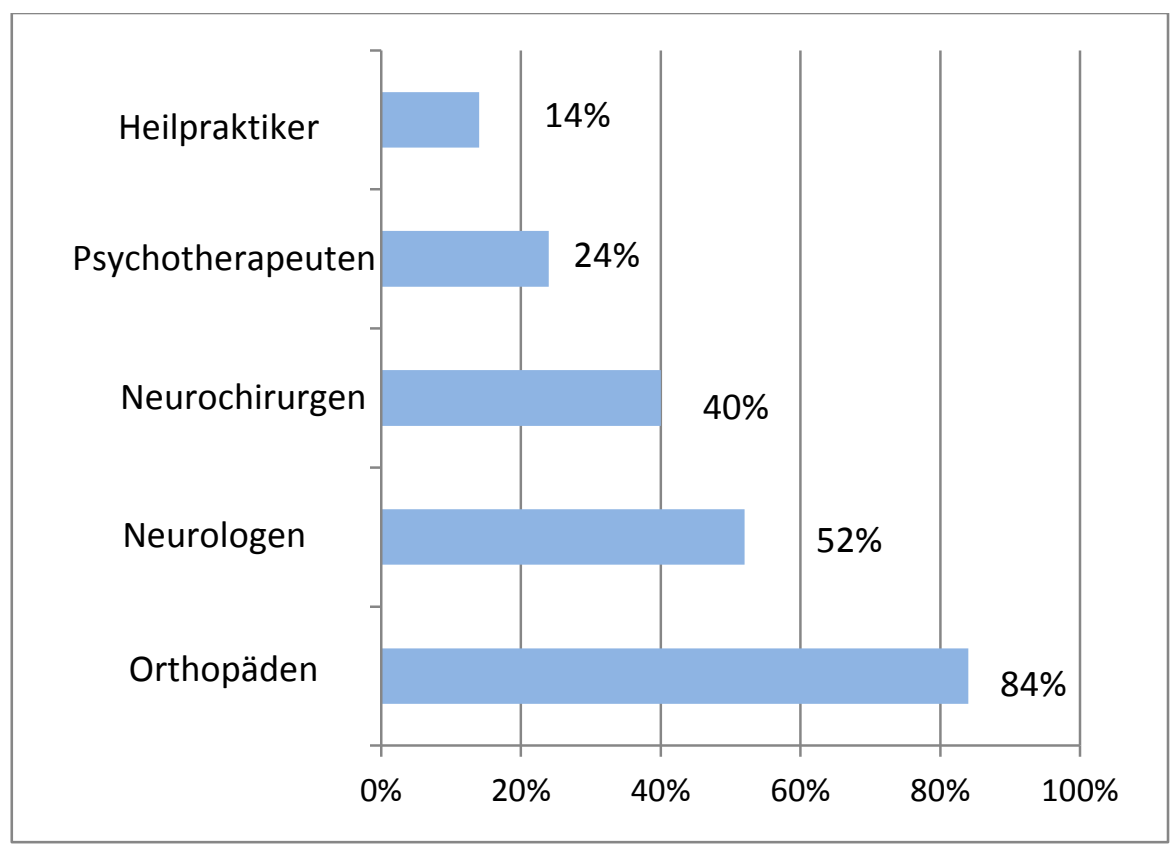

Abb. 14

Weiterbehandelnde Arztgruppen

Im Folgenden werden die einzelnen ärztlichen Fachrichtungen bzgl. der Besuchsfrequenz und der jeweiligen Therapiemaßnahmen gesondert betrachtet.

\subsubsection{Orthopädie}

Die Fachrichtung der Orthopädie zeichnet sich durch durchschnittlich 4,7 jährliche Termine aus (37 gewertete Antworten, Spannweite 2-15 Termine jährlich; SD 3,5).

Die befragten Patienten gaben an, in den orthopädischen Praxen folgende Therapien erhalten zu haben (s. auch Abb. 15):

$58 \%$ wurde Physiotherapie verschrieben.

45\% wurden mit Hilfe von Manipulationen (z.B. „Einrenken“) behandelt.

$44 \%$ bekamen Injektionen in den Rücken, in $84 \%$ erfolgte dies ohne Bildgebung.

35\% bekamen ein TENS-Gerät oder Reizstrom verabreicht.

$20 \%$ wurden Einlagen für die Schuhe verschrieben. 


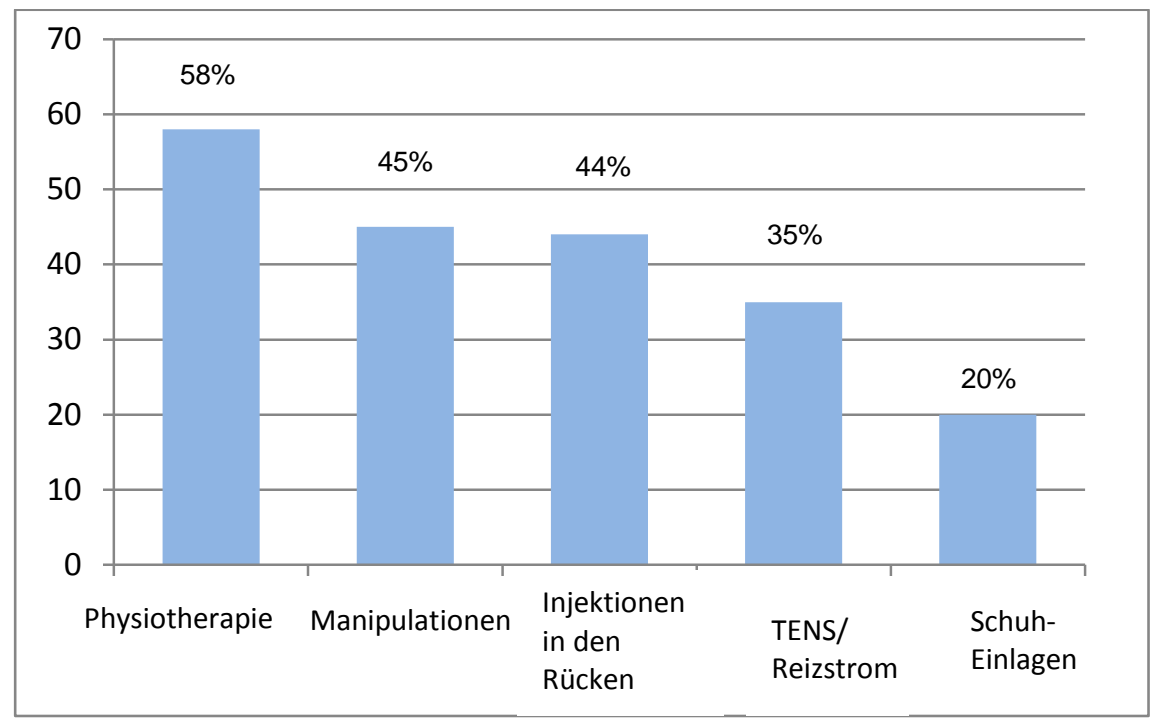

Abb. 15

In orthopädischen Praxen durchgeführte/ verordnete Therapien

44\% der befragten Patienten gaben an, dass Ratschläge zur Intensivierung der körperlichen Aktivität von Orthopäden gegeben wurden.

\subsubsection{Neurologie}

Ein deutliches Problem bei der Befragung ergab sich bei der Unterscheidung von Neurochirurgen und Neurologen. Die Trennung beider Fachgebiete war für manche Patienten nur schwer möglich. Patienten, bei denen aufgrund ihrer Antworten eindeutig geschlossen werden konnte, dass sie von einem Neurologen behandelt worden waren (18 gewertete Antworten), hatten laut ihrer Angaben bei diesem Facharzt insgesamt durchschnittlich 2,4 Termine (Spannweite 1-6 Termine; SD 1,73). Die meisten Neurologen-Kontakte waren jedoch einmalige Besuche, bei denen z.B. die Nervenleitgeschwindigkeit überprüft wurde. In 78\% der Fälle erfolgte überhaupt keine Therapie, ansonsten seien ausschließlich Schmerzmittel verordnet worden. 


\subsubsection{Neurochirurgie}

Mit durchschnittlich insgesamt 4,6 Terminen (20 gewertete Antworten, Spannweite 1-10 Termine; SD 2,98) pro Patient ist diese Disziplin bei unserer befragten Patientenstichprobe bzgl. der Terminhäufigkeit noch vor den Orthopäden platziert (s. aber Einschränkung oben).

Bzgl. der durchgeführten Behandlungen gaben 58\% unserer befragten Patienten an, Injektionen in den Rücken bekommen zu haben (Vgl. Orthopädie: 44\%), davon 2/3 (67\%) mit Bildgebung.

Insgesamt haben 23 von 26 Patienten bei den Neurochirurgen entweder eine Operation (30\%) oder Injektionen in den Rücken (44\%) oder beides bekommen (26\%). 3 Patienten wurden ausschließlich mit Medikamenten oder Physiotherapie behandelt (s. Abb. 16). Weiterhin zu beachten ist, dass beim Neurochirurgen in einer Vielzahl der Fälle die Injektionen mit Bildgebung stattfinden (67\%), während beim Orthopäden die Injektionen ohne Bildgebung dominieren (in 84\% der Fälle).

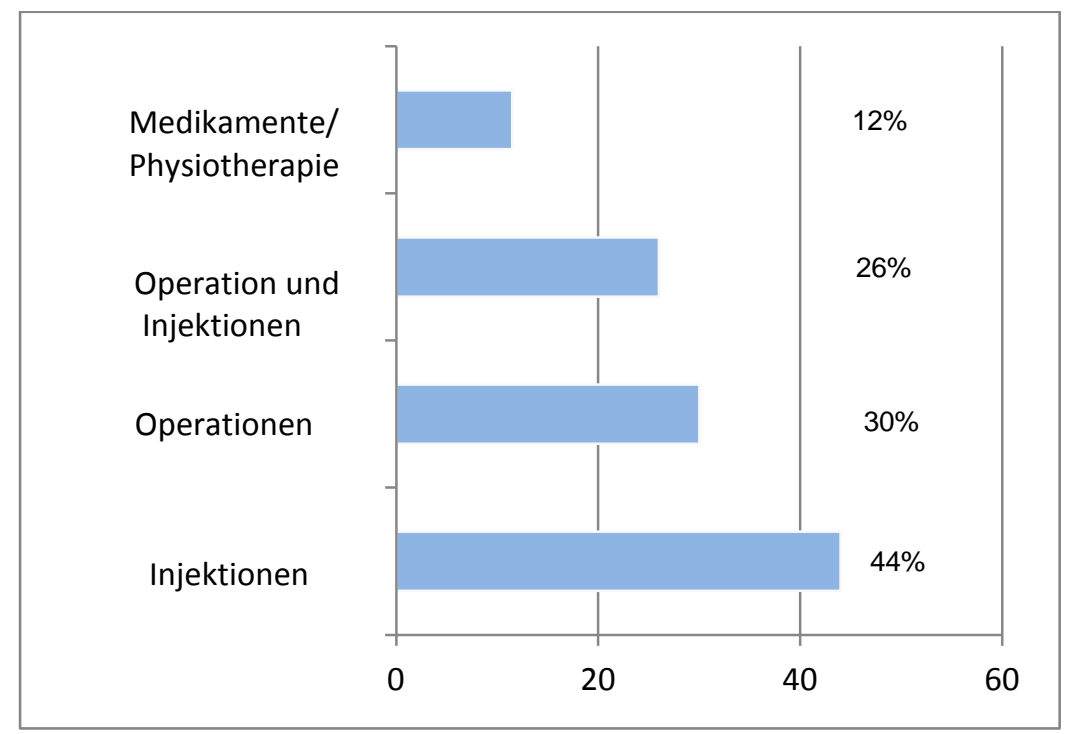

Abb. 16

Neurochirurgische Therapiemaßnahmen 


\subsubsection{Sonstige}

Sehr selten aufgesucht, aber mit der höchsten durchschnittlichen Anzahl der wahrgenommenen Termine sind die Heilpraktiker zu nennen. 14\% der Patienten waren bei einem Heilpraktiker in Behandlung. Mit durchschnittlich 5,7 Terminen pro Patient (Spannweite 1-15 Termine; SD 4,57) führt diese Berufsgruppe die Spitze der Behandlungsanzahlen an.

\subsection{2 Ärztewechsel}

Bei einem Beschwerdebild, dessen Symptomatik sich sehr häufig über einen langen Zeitraum erstreckt, sind, wie oben erwähnt, oft viele therapeutische Berufsgruppen involviert. Es kommt häufig auch zu Therapeutenwechseln.

In über der Hälfte der betrachteten Fälle (52\%) wechselten die Patienten ihren Arzt bzw. ihre Ärzte im Laufe des Krankheitsprozesses. Anlass dafür war in 77\% Unzufriedenheit mit dem Arzt/ seiner Praxis. Gründe für die Unzufriedenheit waren jedoch oftmals nicht die ihrer Meinung nach mangelnde Kompetenz des Arztes, sondern strukturelle Probleme wie zu lange Wartezeiten bei der Terminvergabe, zu volle Wartezimmer oder zu wenig Zeit für das Vorbringen ihrer Anliegen.19\% der Befragten mussten aufgrund eines Wohnortwechsels den Arzt wechseln. 4\% wollten wegen des Wunsches nach Zweit-Meinung einen anderen Arzt derselben Fachrichtung einbeziehen. 


\subsubsection{Informationsweitergabe}

\subsubsection{Motivation zu körperlicher Aktivität und Teilnahme an lokalen Bewegungsprogrammen}

74\% der befragten Patienten gaben im Interview an, innerhalb des ärztlichen Gespräches motiviert worden zu sein, ihre körperlichen Leistungen zu intensivieren. Diese Anregung sei vor allem von den Orthopäden ausgegangen (in $46 \%$ der Fälle); bzgl. des Hausarztes erinnern sich ein Viertel der Patienten (27\%) daran, diesbezüglich motiviert worden zu sein. Die Erwartung, dass vor allem in Rehabilitationseinrichtungen verstärkt zum Sport aufgefordert wird, kann anhand unserer Patienten-Antworten nicht bestätigt werden. Rehabilitationseinrichtungen und Neurochirurgen sind in diese Thematik offensichtlich kaum involviert.

Wir wollten außerdem wissen, inwieweit Ärzte über lokale Angebote von Bewegungsprogrammen (z.B. im nächstgelegenen Fitnessstudio) informiert sind und ob sie dazu Empfehlungen aussprechen. Dies erfolgte jedoch nur in 28\% der Fälle.

\subsubsection{Vermittlung von Hintergrundwissen/ Edukation}

Im Rahmen unseres Gespräches gaben ca. die Hälfte der Patienten (52\%) an, ihrer Meinung nach adäquat über die Ursachen, die verschiedenen Formen sowie Behandlungsmöglichkeiten von Rückenschmerzen aufgeklärt worden zu sein. In ungefähr 2/3 der Fälle (62\%) geschah dies erst im weit fortgeschrittenen Stadium der Erkrankung.

\subsubsection{Zufriedenheit mit den behandelnden Arztgruppen}

Zur Beurteilung der Zufriedenheit der Patienten mit der Behandlung gaben wir eine Skala von 1-5 vor (1: überhaupt nicht zufrieden, 5: hervorragend); (Gesamtvergleich s. Tab. 4). 
Bzgl. der Orthopäden (s. Abb. 17) wird über eine durchschnittliche Zufriedenheit von 3,3 berichtet (36 gewertete Antworten, Spannweite 1-5; SD 1,32).

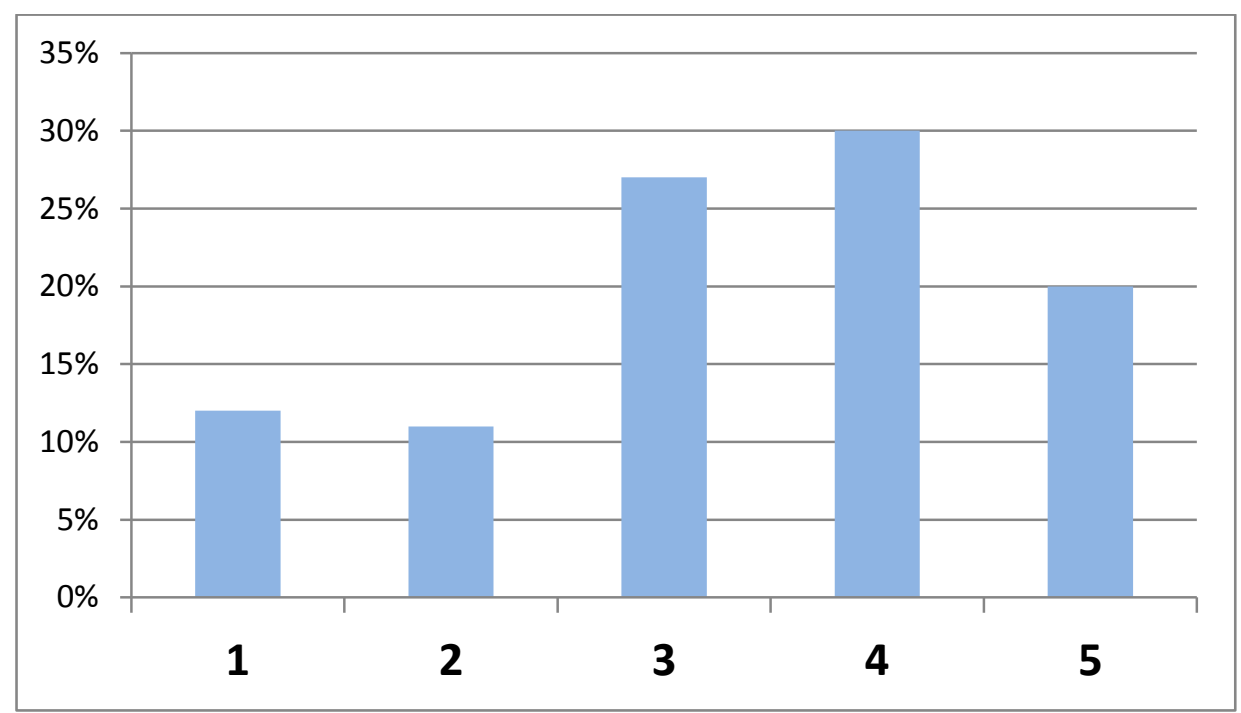

Abb. 17

Zufriedenheit mit orthopädischer Behandlung (Noten von 1-5)

Die Patienten, die mindestens zweimal einen Neurologen kontaktiert hatten (11 Patienten), gaben eine Zufriedenheit von durchschnittlich 3,1 Punkten (Spannweite 1-5; SD 1,03) an.

Bei den Neurochirurgen vergaben ca. die Hälfte aller Befragten ( $46 \%$ bei 26 gewerteten Antworten) die Bestnote 5 (s. Abb. 18). Im Mittel wird eine Note von 3,8 erreicht. 


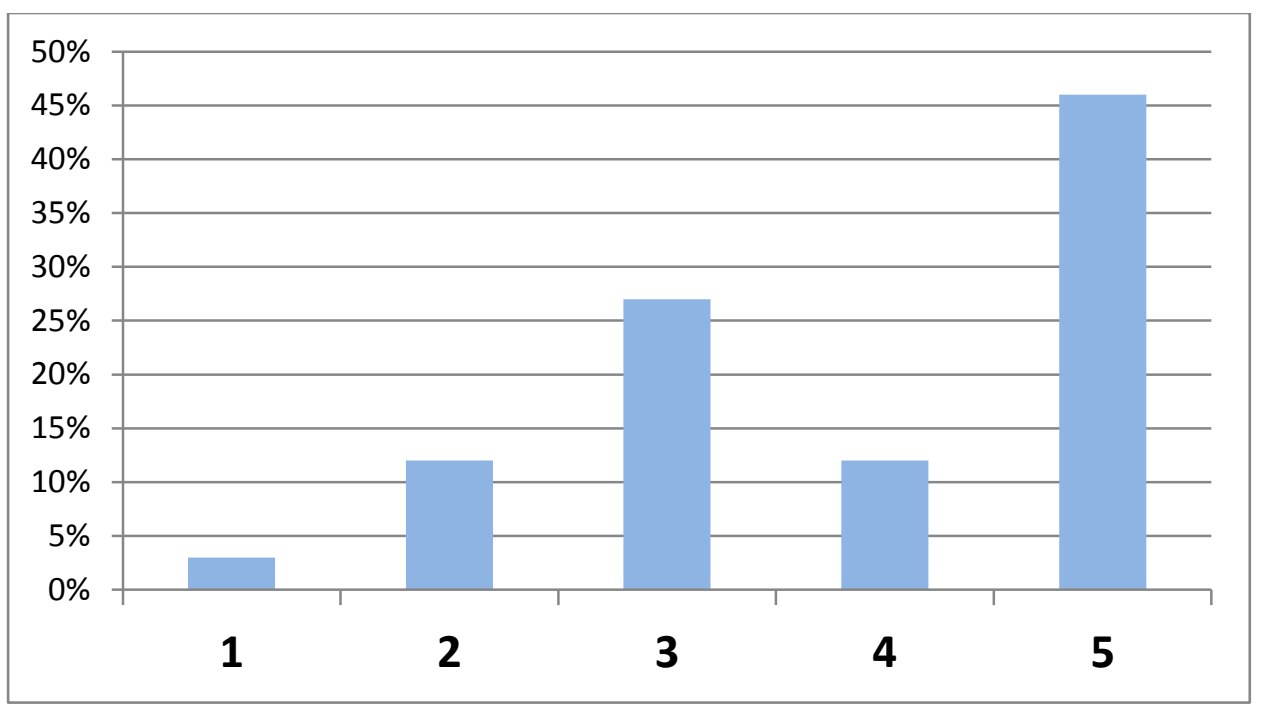

Abb. 18

Zufriedenheit mit neurochirurgischer Behandlung (Noten 1-5)

Bezüglich der Heilpraktiker ergab sich eine Zufriedenheits-Gesamtnote von durchschnittlich 3 (Spannweite 1-5; SD 1,29).

\begin{tabular}{|l|c|}
\hline Arztgruppe & $\begin{array}{c}\text { Durchschnittliche Zufriedenheit } \\
\text { Gesamtnote: Skala 1-5, Bestnote 5 }\end{array}$ \\
\hline Orthopäden & 3,3 \\
\hline Neurologen & 3,1 \\
\hline Neurochirurgen & 3,8 \\
\hline Heilpraktiker & 3,0 \\
\hline
\end{tabular}

Tab. 4

Vergleich der Zufriedenheits-Werte

mit allen beteiligten Facharzt-Gruppen 


\section{Diskussion der Ergebnisse, Vergleich mit der aktuellen Leitlinie}

\subsection{Zusammenfassung der Fragestellung}

Leitlinien entsprechen unter Berücksichtigung von Evidenz-basierter Medizin „dem besten Stand der Erkenntnisse aus Wissenschaft und Praxis“ (Nationale Versorgungsleitlinie Kreuzschmerz" Version 1.2. 2011, S. 9). Angesprochen sind sowohl Ärzte und nicht-ärztliche Berufsgruppen, die direkt an der Versorgung erkrankter Personen beteiligt sind, als auch Betroffene und ihre Angehörigen selbst. Durch Aufstellen von Leitlinien soll die Kooperation zwischen den verschiedenen Behandlungssektoren (ambulant, stationär, rehabilitativ) sowie die Zusammenarbeit von Primär- und Sekundärversorgung erleichtert werden („Nationale Versorgungsleitlinie Kreuzschmerz" Version 1.2. 2011).

Leitlinien für die Behandlung von (nicht-spezifischen) Rückenschmerzen gibt es seit längerer Zeit (z.B. DEGAM-Leitlinien, Nationale Versorgungsleitlinien, DGOOCLeitlinien, DGS-Leitlinien). Für unsere Studie diente die "Nationale Versorgungsleitlinie Kreuzschmerz Version 1.2. 2011" als Grundlage. Diese wurde zwischen August 2006 und 2010 erarbeitet und zuerst im November 2010 veröffentlicht. Durch stetige Überarbeitung und Weiterentwicklung entstanden bis heute 4 Versionen (Version 4 im August 2013 veröffentlicht) (http://www.versorgungsleitlinien.de/themen/kreuzschmerz, September 2013).

Die Empfehlungen der genannten LL unterscheiden sich in wesentlichen Punkten nicht. Aufgabe soll es u.a. sein, die Behandler für eine frühzeitige Erkennung und Wahrnehmung möglicher Chronifizierungsrisikofaktoren zu sensibilisieren. Weiterhin soll die Leitlinie dazu beitragen, dass diagnostische Maßnahmen, die weitgehend ohne therapeutische Konsequenzen bleiben würden, verringert werden, und dass ein größeres Augenmerk auf die Vermeidung unnötiger und obsoleter Maßnahmen gerichtet wird. Aufgabe ist außerdem die Konzentration der Therapiemaßnahmen auf Behandlungen, die eine zügige Rückkehr zum alltäglichen Leben bewirken. 
Wie im Kapitel 3 beschrieben, haben verschiedene Studien (Feuerstein et al. 2006, Schulte et al. 2009 sowie Chenot et al. 2009a) gezeigt, dass es deutliche Defizite im Bereich der Behandlung von akuten sowie chronischen Kreuzschmerzen gibt und dass die Leitlinien-Adhärenz oftmals als ungenügend bezeichnet werden muss.

Probleme bei der Umsetzung von Leitlinien entstehen oft nicht auf Grundlage des NichtWissens. Oft sind laut Schers et al. (2001) beide Seiten (Ärzte und Patienten) gut informiert, dennoch würden viele Patienten eine simple Diagnose und einen einfachen Ratschlag vom Arzt erwarten, anstelle von neuesten veröffentlichten leitliniengerechten Behandlungsmethoden. Gründe für die Non-Adherence seien vor allem vorausgehende Erfahrungen der Patienten und das Beharren der Ärzte auf ihre individuellen preferierten Behandlungsmaßnahmen.

Grol (2001) beschreibt, dass die Leitlinien-Empfehlungen zu $67 \%$ befolgt werden würden. Zu dieser Einschätzung kam Grol (2001), nachdem er innerhalb eines Beobachtungszeitraums von 10 Jahren 70 verschiedene niederländische evidenzbasierte Leitlinien der Familienmedizin auf ihre Umsetzung überprüfte. Dabei unterschied sich die Befolgung der Leitlinien sowohl innerhalb der Ärzte als auch innerhalb der Leitlinien selber deutlich. Die Grundlage für eine praktikable und erfolgreich angenommene Leitlinie sei seiner Meinung nach eine vorab gute Planung der Leitlinien-Einführung, bevorzugt mit Pilotierung.

Die Behandlung von nicht-spezifischen chronischen Rückenschmerzen stellt ein besonderes Problem dar. Wie aus der Literatur bekannt ist (z.B. in Pfingsten, Hildebrandt 2010), zeigen sich mögliche Faktoren für die Entwicklung eines chronischen Krankheitsverlaufs oftmals schon zu Beginn der Erkrankung, weswegen dem Verhalten des Erstbehandlers eine enorme Bedeutung zukommt. Über dieses Verhalten ist jedoch noch relativ wenig bekannt und es macht Sinn, genauer zu untersuchen, ob die Empfehlungen der Leitlinie in diesem entscheidenden Zeitraum des Krankheitsgeschehens adäquat umgesetzt werden.

In schmerztherapeutischen Einrichtungen werden vorrangig chronische Schmerzpatienten behandelt. Diese haben oftmals eine lange Krankheitsgeschichte und bereits vielfältige Behandlungen hinter sich. Aus den Erfahrungen mit diesem 
Patientenklientel und den Kenntnissen über die Behandlungsvorgeschichte lässt sich die Hypothese aufstellen, dass ihre Chronifizierung (mindestens zu einem gewissen Anteil bzw. bei einem Teil der Betroffenen) auch deswegen eingetreten ist, weil es im Vorfeld in den diagnostischen Maßnahmen bzw. den durchgeführten therapeutischen Interventionen zu Versäumnissen bzw. sogar Fehlern gekommen ist und Leitlinienempfehlungen nicht ausreichend befolgt wurden. Daher war es das Ziel der vorliegenden Studie, Patienten mit chronischen Rückenschmerzen standardisiert und chronologisch zu ihren Vorbehandlungen zu befragen, die vom Zeitpunkt der Erstkonsultation eines Arztes bis zur Vorstellung in einer spezialisierten multimodal ausgerichteten Schmerzklinik stattfanden, sowie anhand der Analyse der Ergebnisse deren Leitlinien-Kompatibilität zu überprüfen. Dabei lag unser Schwerpunkt vor allem auf den diagnostischen Maßnahmen (Ablauf des Erstkontaktes, FacharztÜberweisungen, Bildgebung etc.).

Aus einer genaueren Analyse der Schwachstellen im Versorgungssystem und der Kenntnis der Defizite erhoffen wir uns eine zukünftige Verbesserung der Versorgung, so dass eine Chronifizierung der Beschwerden mit den ausgesprochen nachteiligen Auswirkungen für die Betroffenen wie auch für das Gesundheitssystem in Zukunft besser vermieden werden kann.

Um dieses Ziel zu erreichen, wurde die Behandlungsvorgeschichte von 50 konsekutiven Patienten der Göttinger Schmerzklinik, bei denen ein chronischer nicht-spezifischer Kreuzschmerz diagnostiziert worden war, genauer analysiert. Anhand der vorliegenden Befunde, der Ergebnisse des standardmäßig eingesetzten Deutschen Schmerzfragebogens und einer standardisierten Befragung wurden die Schmerzvorgeschichte, die Behandlungswege, das Überweisungsverhalten, sowie diagnostische und therapeutische Maßnahmen erfasst.

Die Patienten wurden in einem Zeitraum von Oktober 2009 bis September 2010 mit einem standardisierten Fragenkatalog befragt. Auf Grundlage dieses Fragebogens erhielten wir Aufschluss über Alter und Geschlecht unserer Patienten, deren BMI, Personenstand, Schulabschluss und momentaner beruflicher Situation, laufenden 
Rentenverfahren, MdE und GdB sowie die Schmerzintensität und deren Einordnung im MPSS. Die Befragung fand i.d.R. nach dem ersten Arztkontakt statt, in dem die Patienten über die Studie aufgeklärt und um Teilnahme gebeten wurden.

\subsection{Patientenbeschreibung}

In die Studie eingeschlossen wurden 50 Patienten, 26 Männer und 24 Frauen im Alter zwischen 23 und 63 Jahren.

Unsere Analyse basierte auf den Angaben einer hoch chronifizierten Patientengruppe, die sich durch eine umfangreiche Vorgeschichte, lange Krankheitsdauer mit vielfältigen Arztkontakten, multiplen diagnostischen und therapeutischen Maßnahmen sowie langer Arbeitsunfähigkeit auszeichnete. Sie repräsentierten mit diesen Merkmalen das typische Klientel einer Schmerzklinik.

Nach Auswertung unserer Ergebnisse zeigt sich ein möglicher Zusammenhang von Prävalenz und den Patienten-bezogenen Merkmalen Übergewicht, Sozialstatus und Arbeitsfähigkeit:

- Übergewicht:

Die Befragten zeigten einen durchschnittlichen BMI von 28. Ca. 2/3 der Befragten hatten mindestens einen BMI von 25. In der Literatur beschreiben z.B. Heuch et al. sowohl im Jahr 2010 als auch im Jahr 2012 eine hohe Assoziation von Übergewicht und Rückenschmerzprävalenz. Insgesamt scheint es, dass ein erhöhter Body Maß Index ein gewisses Risiko für das Auftreten von chronischen nicht-spezifischen Rückenschmerzen birgt, dennoch beschreiben Fahland et al. (2011), dass bisher ein unmittelbarer Zusammenhang von Übergewicht und chronischen nicht-spezifischen Rückenschmerzen nicht ausreichend belegt ist. Als Merkmal einer schlechten Selbstfürsorge steht Übergewicht möglicherweise nur in mittelbarem Zusammenhang zu Rückenschmerzen. 
- Sozialstatus:

Wie in Kapitel 5.1.2 dargestellt, findet sich bei unseren Befragten die größte Prävalenz der Rückenschmerzen bei einem niedrigen bis mittleren Bildungsniveau (10-11 Schuljahre), wohingegen die in unserer Befragung von Rückenschmerz betroffenen Patienten mit 12 oder mehr Bildungsjahren nur 1/10 der Betroffenen ausmachten. Die größere Prävalenz der Rückenschmerzen bei Personen mit einem Haupt- (34\%) oder Realschulabschluss (50\%) lässt die Frage aufkommen, ob möglicherweise ein Zusammenhang zwischen der Kreuzschmerz-Prävalenz und einem mittleren bis niedrigerem Sozialstatus besteht.

Diese Thematik ist in der Literatur in den letzten Jahren oftmals untersucht und diskutiert worden. Dionne et al. (2001) untersuchten diese potentielle Assoziation zwischen einem geringen sozioökonomischen Status und Rückenschmerzen. Sie kamen zu dem Ergebnis, dass Bildung potentiell in dreifacher Hinsicht mit dem Auftreten von Rückenschmerzen in Verbindung steht:

- $\quad$ zum einen als Prädiktor für die Frequenz von Rückenschmerzen (Inzidenz, Rekurrenz, Prävalenz),

- $\quad$ als Prädiktor für das Outcome nach einer Rückenschmerzepisode (bezüglich Arbeitsplatz- und Freizeit-Interferenzen und Gesundheitsvorsorge) und

- $\quad$ als Prädiktor für das Outcome nach chirurgischen und rehabilitativen Interventionen nach einem Rückenschmerzereignis.

Zusammenhänge sind in der Weise vorstellbar, dass Menschen mit geringerem Bildungsniveau möglicherweise einen geringeren Zugang zu spezialisierten medizinischen Interventionen haben als diejenigen mit höherer Bildung. Sie warten möglicherweise länger, bis sie bei Beschwerden einen Arzt konsultieren und zeichnen sich eventuell durch eine geringere Compliance bzgl. der therapeutischen Empfehlungen aus. Laut Dionne et al. (2001) belegen jedoch mehrere Studien, dass Rückenschmerzpatienten mit geringerer Bildung mit einer höheren Frequenz Ärzte konsultieren und öfter hospitalisiert sind. Fest steht, dass Menschen mit geringerem sozioökonomischen Status und gering bezahlten Beschäftigungsverhältnissen öfter körperlich belastendere Tätigkeiten ausüben. Außerdem kehren laut Reisbord \& Greenland (1985) Menschen mit niedrigerem sozioökonomischem Status aus Sorge um 
ihren Arbeitsplatz schneller nach einer durchgemachten Rückenschmerzepisode zum Arbeitsalltag und körperlich belastenden Tätigkeiten zurück, auch wenn die Symptome noch persistieren. Hier zeigt sich ein deutlicher Zwiespalt: laut Leitlinie wird eine zügige Rückkehr zum Arbeitsplatz angestrebt, um einer Chronifizierung vorzubeugen, andererseits sollte aber auch eine ausreichende Genesungszeit eingehalten werden. Wie eine zu frühe Rückkehr definiert werden sollte, bleibt offen; die Grenzen zu einem nicht genügend „auskuriertem“ Problem sind fließend. Prinzipiell stellen bestehende (nicht-spezifische) Rückenbeschwerden keine Kontraindikation für eine schnelle Rückkehr zum Arbeitsalltag dar, solange die Symptome rückläufig sind und idealerweise eine gewisse Flexibilität bei den Arbeitshaltungen/-belastungen möglich ist. Anstelle der Aussage, dass generell zügig zum Arbeitsplatz zurückgekehrt werden sollte, wäre möglicherweise der Zusatz sinnvoll, dass solange Patienten trotz noch bestehender Schmerzen eine Besserung spüren, eine (ggf. flexibel gestaltete) Rückkehr zum Arbeitsplatz angestrebt werden sollte. Erfahrungsgemäß sind derartige flexible Gestaltungen der Rückkehr an den Arbeitsplatz in größeren Firmen/ Einrichtungen, die über eine mitwirkende betriebsärztliche Abteilung verfügen, einfacher umzusetzen.

Franks \& Boisseau (1980) sehen zwischen Bildungsniveau und Rückenschmerzen allenfalls einen mittelbaren Zusammenhang, indem Personen mit höherem Bildungsniveau eine bessere und gesündere Lebensführung und ein größeres Bewusstsein für Gesundheitsrisiken haben, als es ihrer Meinung nach Menschen mit niedrigerem Bildungsniveau aufweisen.

Man muss zusammengefasst davon ausgehen, dass es keine einfache kausale Assoziation zwischen geringer Bildung und hoher Kreuzschmerzprävalenz gibt („Educational status may be a "marker" for other factors involved in the aetiology or natural history of the disease." Dionne et al. 2001, S. 466).

Nach jetzigem Erkenntnisstand lässt sich zusammenfassend nicht davon ausgehen, dass Personen mit geringerer Bildung bzgl. der Erstmanifestation öfter an Rückenschmerzen leiden, sondern Bildung vor allem bzgl. des weiteren Verlaufes der Erkrankung relevant wird. Dies betrifft also den Umgang der Betroffenen mit ihrer Erkrankung sowie ihre Fähigkeit, auf (körperliche) Beeinträchtigungen zu reagieren und 
ggf. auch ihr Verhalten zu ändern. "The evidence is stronger for an effect of education on the duration and/ or recurrence of back pain episodes than for an effect of education on the incidence of back pain" (Dionne et al. 2001, S. 466). Als Konsequenz aus diesem Befund ist die Forderung zu stellen, dass z.B. edukative Inhalte "bildungsgerecht" formuliert sein sollten. Präventive Angebote sollten dahingehend geprüft werden, ob sie auch von Menschen mit niedrigerem Berufsstand wirklich in Anspruch genommen und umgesetzt werden können (z.B. Flexibilität der Angebote, so dass Patienten z.B. mit Schichtarbeit teilnehmen können).

- Beruf/ Arbeitsfähigkeit:

64\% unserer Befragten beklagten in den letzten 3 Jahren mindestens 3 Monate rückenschmerzbedingten Arbeitsausfall. Dabei fällt auf, dass Männer durchschnittlich 11 Monate aufgrund der Kreuzschmerzen nicht am Berufsleben teilnehmen konnten, wohingegen Frauen nur 6 Monate im Durchschnitt ausfielen.

Dies lässt sich möglicherweise dadurch erklären, dass die an unserer Studie teilnehmenden Männer oftmals körperlich belastende als auch den Witterungsbedingungen ausgesetzte Berufe ausübten und schlichtweg diese Umstände eine zügigere Rückkehr zum Arbeitsplatz verzögerten, wohingegen die Frauen unserer Studie oftmals administrative Berufe ausübten, die sich mit bestehenden Schmerzen möglicherweise leichter ausüben ließen.

Die Zahlen verdeutlichen, dass Rückenschmerzen bei den betrachteten Patienten erhebliche Auswirkungen i.S. einer Beeinträchtigung der Erwerbsfähigkeit haben. Auch Pfingsten et al. (2011) beschreiben, dass das "häufigste sozialmedizinische Problem“ (Pfingsten et al. 2011, S. 107) bei Rückenschmerzen die Arbeitsunfähigkeit ist. Oft würden die Patienten der Meinung sein, dass ihre Rückenschmerzen von der Arbeitstätigkeit herrühren bzw. dass sie bei Wiederaufnahme der Tätigkeit verschlimmert werden könnten. „Der Wunsch nach Krankschreibung geht in den meisten Fällen vom Patienten aus und ist beim Arztbesuch häufig der wichtigste Konsultationsgrund“" (Pfingsten et al. 2011, S. 107). Die Beurteilung/ Einschätzung der beruflichen Leistungsfähigkeit des individuellen Patienten vor dem Hintergrund der vorhandenen Berufsbelastung ist von Seiten des Arztes nicht immer einfach. Es gibt 
durchaus Evidenz dafür, dass ungünstige Körperhaltungen während der Arbeit zu Rückenschmerzen führen können, allerdings kann nicht von einem einfachen biomechanischem Zusammenhang zwischen Art der Körperhaltung und dem Auftreten von Rückenschmerzen gesprochen werden (Pfingsten et al. 2011). Hier handelt es sich eher um ein komplexes Phänomen, in das insbesondere subjektive Bewertungen der Betroffenen über die Schwere ihrer Arbeitsbelastung Eingang finden. Diese „subjektive“ Schwere muss mit den objektiv gegebenen Bedingungen nicht unbedingt in Einklang stehen.

Arbeitszufriedenheit wird oft als einer der wichtigsten Prädiktoren für das Auftreten von Rückenschmerzen genannt. Arbeitsunzufriedenheit führt laut Pfingsten et al. (2011) zu einer subjektiven Überschätzung der objektiven Arbeitsbelastung. Es gibt deutliche Hinweise dafür, dass psychologische Einflussfaktoren wie Zeitdruck, das Erleben starker Kontrolle und ein geringes Autonomie-Erleben im Beruf das subjektive Beeinträchtigungserleben erheblich beeinflussen. In diesen Fällen ist die Veränderung der Arbeitsbedingungen (Verhältnisprävention), z.B. über ergonomische Gestaltung oder Veränderungen der Arbeitsmittel und -umwelt, weniger geeignet, um das Auftreten bzw. den Verlauf von Rückenschmerzen zu beeinflussen. Wichtiger erscheint dann die Verhaltensprävention. Diese betrifft das Verhalten jedes einzelnen Arbeitnehmers am Arbeitsplatz. Die Verhaltensprävention beinhaltet einerseits Aufklärungsmaßnahmen, die beim Einzelnen zu "gesundheitsfördernden Verhaltensweisen“ beitragen sollen, andererseits vermittelt sie Bewältigungstechniken für Verhaltensänderungen, z.B. Bewegungsprogramme und Raucher-Entwöhnungs-Programme (http://www.arbeitssicherheit.de/de/html/lexikon/224/) (2013). Auch bestimmte Charakteristika des jeweiligen Berufs (z.B. viel sitzende Tätigkeiten) sollen ausgeglichen werden (Hildebrandt, Pfingsten 2011).

Um dem therapeutischen Ziel (Wiederaufnahme der beruflichen Tätigkeit) zu entsprechen, erscheint die Verhaltensprävention von größerer Bedeutung. Diese betrifft das Verhalten jedes einzelnen Arbeitnehmers am Arbeitsplatz: einerseits Aufklärungsmaßnahmen, die beim Einzelnen zu "gesundheitsfördernden Verhaltensweisen" beitragen sollen, andererseits die Vermittlung von Bewältigungstechniken für Verhaltensänderungen (z.B. Bewegungsprogramme, 
Stressbewältigung, Training sozialer Kompetenz). Der Umgang mit vorhandenen beruflichen Belastungssituationen soll anhand von gesundheitsfördernden Strategien entwickelt und unterstützt werden. Die Patienten sollen lernen ihre Arbeitsabläufe selbst zu regulieren. Auch perfektionistische Tendenzen und überhöhte Ansprüche sollen relativiert werden. Ein weiteres Ziel ist außerdem den Arbeitnehmern eine Work-LifeBalance zu vermitteln: so soll auch eine gewisse Distanzierungsfähigkeit vom Beruf erlernt werden (Bethge 2010).

\subsection{Hauptergebnisse der Befragung und Untersuchung auf ihre Leitlinienkonformität}

Im folgenden Abschnitt werden die Hauptergebnisse der standardisierten Befragung dargestellt und auf ihre Leitlinienkonformität geprüft. Dieses Kapitel ist in 3 Bereiche unterteilt:

- Merkmale der Diagnostik

- Merkmale der Behandlung

- Weitere Ergebnisse

\section{Merkmale der Diagnostik}

- $\quad$ Diagnostik durch Hausärzte und Orthopäden, zweifelhaft geregelte Primär- und Sekundärversorgungsstruktur

Fast 2/3 aller Patienten (63\%) gaben an, nach erstmaligem Auftreten der Beschwerden (so wie es das Prinzip der primärärztlichen Grundversorgung vorsieht) zuerst ihren Hausarzt konsultiert zu haben.

Bei 24\% unserer Befragten fand die Erstkonsultation bei einem Orthopäden statt. Insgesamt war bei $84 \%$ aller Patienten (mindestens) ein Orthopäde in die Behandlung einbezogen. Diese stellen somit die am meisten konsultierte Facharzt-Gruppe dar.

Die Orthopäden waren laut unserer Studie wesentlich an der Patienten-Motivierung (bezüglich Intensivierung von sportlichen Aktivitäten) beteiligt und diejenigen, die am häufigsten die potentiellen Risikofaktoren für eine Chronifizierung (yellow flags) anamnestisch erfragt haben sollen. 
Gründe hierfür könnten sein, dass Hausärzte meist sehr gut über den privaten Hintergrund ihrer Patienten Bescheid wissen, sodass sie deswegen möglicherweise weniger Notwendigkeit gesehen haben, bei Konsultationen repetitierend nach dem Familien- und Berufsstatus zu fragen, wohingegen die Orthopäden wenig Hintergrundinformationen im Vorab erhalten und deshalb explizierter danach fragen müssen. Auch die Ergebnisse unserer Befragung (Meinung der Patienten, s. S. 101) lassen vermuten, dass Hausärzte die yellow flags nicht aus Ignoranz nicht erfragt haben, sondern aufgrund der nicht gesehenen Notwendigkeit, weil innen die Lebensumstände der Patienten schon viele Jahre bekannt sind.

Erwartungsgemäß verschrieben die Orthopäden jedoch deutlich mehr bildgebende Diagnostik (58\%) beim ersten Arztkontakt als die Hausärzte (41\%).

Die Verordnung von Physiotherapie ging nahezu gleichermaßen sowohl von den Hausärzten (47\%) als von den Orthopäden aus (45\%).

Die frühe Einbeziehung von Fachärzten und die Involvierung meist mehrerer Ärzte derselben Fachrichtung (teilweise drei verschiedene Orthopäden gleichzeitig) deutet an, dass es bei der Behandlung von Kreuzschmerzen offensichtlich keine geregelte Unterscheidung bzgl. Primär- und Sekundärversorgungsstruktur gibt. Die Ergebnisse unserer Befragung spiegeln damit nicht die Empfehlungen wider, die in der Leitlinie Kreuzschmerz für eine effektive wie gleichzeitig auch kostengünstige Versorgung gegeben werden (Chenot et al. 2009a). Die Leitlinie empfiehlt, zwischen einer "hausärztlichen Primärversorgung" und einer "spezialisierten Sekundärversorgung" (Chenot et al. 2009a, S. 150) zu unterscheiden. In der "Nationalen Versorgungsleitlinie Kreuzschmerz" (Version 1.2., 2011) wird erst bei einer über 2 Wochen andauernden Arbeitsunfähigkeit die Hinzuziehung weiterer Fachdisziplinen empfohlen. Der hohe Anteil derer, die schon beim ersten Arztkontakt einen Spezialisten kontaktieren und somit den Facharzt zu früh aufsuchen, läuft dem Prinzip der sequentiellen Versorgung zuwider. Dadurch entstehen vermutlich unnötige und vermeidbare Kosten, die an anderer Stelle sinnvoller eingesetzt werden könnten.

Insgesamt gesehen beschreiben Hauswaldt et al. (2012) außerdem interessanterweise, dass die Anzahl der Hausarzt-Konsultationen von Patienten mit chronischen Erkrankungen, anders als erwartet, nicht die Frequenz der Hausarzt-Besuche anderer 
Patientengruppen übersteigt. Sie sollen laut Hauswaldt et al. (2012) tendenziell insgesamt sogar weniger Kontakte mit primärversorgenden Institutionen wahrnehmen.

Bei unserer Befragung entstand außerdem der Eindruck, dass es aufgrund der teilweise fehlenden Kommunikation der parallel beteiligten Ärzte untereinander vermutlich zu „Doppel-Diagnostik“ und sich nicht unbedingt ergänzenden therapeutischen Verschreibungen kommt.

\section{- $\quad$ Körperliche Untersuchung oft zu oberflächlich}

Die Grundlage der körperlichen Untersuchung stellt zuallererst die Inspektion des Patienten dar, d.h. die Beurteilung seines Haltungsmusters und des Gangbildes. Weiterhin sollen mögliche Schonhaltungen überprüft und z.B. Skoliosen aufgedeckt werden. Anschließend sollten die Wirbelsäule des entkleideten Patienten gründlich palpiert und auf mögliche Klopfschmerzhaftigkeiten untersucht werden. Außerdem sollten Beweglichkeitsprüfungen (z.B. Inklinations- und Reklinationsübungen) und Wurzeldehnungstests (Lasegue) durchgeführt werden.

Erfreulicherweise können sich 95\% der Befragten daran erinnern, bei ihrer ersten rückenschmerzbedingten Arztkonsultation körperlich untersucht worden zu sein. 83\% von ihnen gaben an, dass sie ihre Kleidung ablegen sollten. Dies beinhaltete jedoch in den meisten Fällen nur eine unvollständige Entkleidung (Oberkörper bis zur Hüfte). Obwohl wie bereits oben erwähnt bei neu aufgetretenen Kreuzschmerzen außerdem eine ausführliche Untersuchung der Wirbelsäule obligatorisch sein sollte, gaben auch hier nur $88 \%$ der Patienten an, dass die Wirbelsäule und paravertebrale Muskulatur palpiert worden sei. Jedoch muss an dieser Stelle nochmals betont werden, dass aufgrund der schon lange zurückliegenden Erstuntersuchung keine eindeutig zuverlässigen Aussagen bezüglich der Erstkonsultation erhoben werden konnten.

Auch war es schwierig adäquate aussagekräftige Antworten zu stattgefundenen Beweglichkeitsprüfungen bzw. dem Lasegue-Test (siehe Kapitel 2.3, Abb. 2) zu erheben. 
Zusammenfassend muss man trotzdem bzgl. der Sorgfalt der körperlichen Untersuchung davon ausgehen, dass dieselbe verbesserungsbedürftig erscheint. Bezogen auf die Angaben der "Nationalen Versorgungsleitlinie Kreuzschmerz" (Version 1.2., 2011) scheint die Qualität der körperlichen Untersuchung wahrscheinlich unzureichend. Das Minimum der Untersuchung sollte zumindest die vollständige Entkleidung des Patienten und die Basis-Untersuchung der Wirbelsäule sein.

- $\quad$ Vernachlässigung psychosozialer Risikofaktoren

Der Erhebung psychosozialer Risikofaktoren scheint trotz ihrer großen Bedeutung für die Chronifizierung, nur eine relativ geringe Beachtung zuteil zu werden. Die Patienten gaben diesbezüglich an, dass sie - wenn überhaupt - lediglich nach dem Beruf gefragt worden seien. Bei nur 1/5 aller Betroffenen wurde laut Patientenaussagen die familiäre Situation erfragt. Der Umgang mit den Schmerzen soll bei niemandem thematisiert worden sein.

Psychosozialen Risikofaktoren kommt in der Chronifizierung von Rückenschmerzen eine erhebliche Bedeutung zu. Diese Bedeutung wird in der NVL an verschiedenen Stellen betont und hat ihren Eingang in die Empfehlungen gefunden: Bei erfolgloser Akutbehandlung über 2 bis 4 Wochen wird die Erhebung der sog. yellow flags als Risikofaktoren für eine Chronifizierung (siehe Kapitel 2.4) empfohlen. Bei Bestehen der Beschwerden für mehr als 4 Wochen trotz leitlinien-gerechter Therapie soll erneut eine psychosoziale Einschätzung erfolgen, sofern dies nicht bei der Eingangsdiagnostik berücksichtigt wurde. Bei bestehendem Verdacht auf eine psychosoziale Beteiligung wird bereits nach 2 Wochen andauerndem nicht-spezifischem Kreuzschmerz die Konsultation eines Psychologen oder Psychotherapeuten angeraten. Bei einer Symptompersistenz von mehr als 6 Wochen sollen nochmals mögliche Chronifizierungs-Risikofaktoren überprüft werden.

All diese Empfehlungen sind jedoch laut unserer Patientenaussagen oftmals nicht ausreichend umgesetzt worden.

Dies mag am Zeitmangel auf Grund der oft knapp bemessenen Konsultationszeit liegen. Insbesondere aufgrund der Tatsache, dass viele Patienten eine stärkere KausalAttribution auf körperliche Symptome haben und psychologische Erklärungen oftmals 
als Angriff auf die „Legitimität“ ihrer Schmerzen interpretieren, ist ein Gespräch darüber zeitintensiv und verlangt eine entsprechende Kommunikationskompetenz. Insofern ist mindestens in einzelnen Fälle davon auszugehen, dass die Vermeidung derartiger Inhalte einen unproblematischeren Kontakt auf beiden Seiten garantiert.

Insgesamt sollten sowohl die Verarbeitung bzw. der Umgang mit den Schmerzen als auch die familiäre und berufliche Situation (besser) thematisiert werden. Helfen könnten hierbei entweder eine standartmässige Erfassung der yellow flags/ Screening Verfahren bei jedem Patienten zu Beginn der Konsultation (z.B. durch einen Fragebogen mit Angaben zu Beruf und Familie ausgefüllt mit Hilfe der zuständigen Arzthelferin) als auch ein vielseitig nutzbarer Frageleitfaden für den Arzt, der bei etwaigen Unsicherheiten zu Hilfe genommen werden könnte.

Einschränkend ist hier jedoch erneut anzuführen, dass die personellen Kapazitäten zur Umsetzung dieser Forderung derzeit nicht ausreichend vorhanden sind (s.o.).

- $\quad$ Zu viel und unnötige Bildgebung

Laut Leitlinie ist eine routinemäßige Bildgebung bei Kreuzschmerzen obsolet. Eine Bildgebung bei der ersten Arztkonsultation sollte nur dann erfolgen, wenn aus der körperlichen Untersuchung bzw. der Anamnese red flags als Warnhinweise für das Vorliegen einer gravierenden körperlichen Pathologie (siehe Kapitel 2.5.1.1, S. 15) erkennbar sind. Die Hälfte der Patienten berichtete, dass schon beim ersten Arztkontakt ein bildgebendes Verfahren veranlasst worden sei.

In Bezug auf die Art der Bildgebung wurden 78\% unserer Patienten in den letzten 3 Jahren mindestens einmal wegen ihres Rückens geröntgt. Ebenfalls $80 \%$ aller Befragten bekamen wegen der Rückenschmerzen in den letzten 3 Jahren mindestens ein MRT, 60\% aller Befragten erhielten in den letzten 3 Jahren mindestens ein CT.

Mit dem Vorliegen der oben genannten gravierenden körperlichen Pathologie ist insgesamt in den weniger häufigen Fällen zu rechnen (Niebling 2004), so dass der Anteil unserer Befragten (51\%), die schon beim ersten Arztbesuch eine Bildgebung erhalten haben, als $\mathrm{zu}$ hoch erscheint. Falls nach 6-wöchiger leitlinienorientierter Behandlung keine Besserung und evtl. sogar eine Progression der Beschwerden eintritt, wird eine einmalige bildgebende Diagnostik empfohlen. „Das in den Leitlinien 
verwendete Zeitkriterium 6 Wochen, innerhalb derer ohne Vorliegen von Warnhinweisen keine Bildgebung empfohlen wird, wird oft missverstanden in dem Sinne, dass nach 6 Wochen (regelhaft) eine Bildgebung zu erfolgen hat" (Chenot 2010, S. 216).

Insgesamt erscheint die Verwendung bildgebender Maßnahmen zu früh und die Anzahl der Bildgebungen unserer befragten Patienten als $z u$ hoch. Owen et al. (1990) berichten, dass auf die Frage, warum Ärzte so oft zu radiologischer Bildgebung tendieren würden, 88\% antworteten, dass sie dies zur Beruhigung ihrer Patienten veranlassten. $78 \%$ antworteten, dass sie sich selber beruhigen und versichern wollten, dass kein ernsthafter pathologischer Befund vorliegt. Überdenkenswert ist demnach, ob nicht auch eine intensivere Aufklärung der Patienten ein zur „Beruhigung“ dienendes bildgebendes Verfahren überflüssig machen könnte.

Laut Deyo \& Diehl (1986) und Deyo et al. (1987) sind viele Patienten der Überzeugung, dass sie eine Bildgebung bräuchten. Dieses Bedürfnis muss im Aufklärungsgespräch berücksichtigt werden. Wenn möglich sollten Patienten davon überzeugt werden, dass nach Auswertung der Befunde aus der körperlichen Untersuchung ein bildgebendes Verfahren nicht notwendig ist.

- $\quad$ Mangelhafte radiologische Befundübermittlung/ Informationsweitergabe

Zwar findet nach Angaben unserer Patienten relativ oft eine Kommunikation des radiologischen Befundes statt, in vielen Fällen wird jedoch nur zwischen den Botschaften „es ist eine deutliche Schädigung des Rückens ersichtlich“ und „es ist soweit alles in Ordnung" unterschieden; $20 \%$ der befragten Patienten gaben an, dass sie gar keine Mitteilung erhalten haben. Dass bei 70\% der Betroffenen eine "deutliche Schädigung" vorlag, ist dabei eher unwahrscheinlich. Wenn man die internationalen Prävalenzzahlen zugrunde legt, dann ist eher davon auszugehen, dass das Verhältnis umgekehrt ist, d.h., dass bei über $70 \%$ eben keine Schädigung Wirbelsäulen-bezogener Strukturen besteht.

Ein weiteres Problem liegt darin, dass Patienten durch Auskünfte über das Vorliegen eines strukturellen Schadens verunsichert bzw. alarmiert werden. Häufig werden die Patienten durch die „klassische“ Diagnose Bandscheibenvorfall irritiert. 50\% der 
Befragten wurde mindestens ein „Bandscheibenvorfall“ radiologisch bescheinigt, der oftmals jedoch asymptomatisch blieb.

Leider war uns ein Abgleich der Patientenaussagen mit den tatsächlich vorliegenden Befunden nicht ausreichend möglich, dennoch stellten wir fest, dass z.B. keine zufriedenstellende Unterscheidung zwischen Protrusio und Prolaps stattgefunden hat.

Bei einer Protrusio kommt es zu einer Vorwölbung der Bandscheibe, beim Prolaps kommt es zum realen „Vorfall“ der Bandscheibe/ des Nucleus pulposus, welcher oftmals zu einem Zerreißen des Anulus fibrosus führt (Müller 2010). Dies ist oftmals die schwerwiegendere Form. Dennoch wurde bei unseren Patienten oft vereinheitlichend von einem Bandscheiben-„,Vorfall“ gesprochen.

Die Auswirkungen negativer Botschaften aus der Röntgen-Befundung sind bei Kendrick et al. (2001) beschrieben. Patienten, die eine radiologische Befundung erhalten hatten, waren zwar zufriedener mit ihrer Behandlung als diejenigen, die keine Bildgebung erhielten, diese Gruppe (mit Bildgebung) hatte aber insgesamt ein schlechteres Outcome und eine höhere Anzahl an Arztkonsultationen als die Kontrollgruppe. Laut Kendrick et al. (2001) könnte einer der Gründe hierfür sein, dass radiologische Untersuchungen die Patienten auf einen schwereren Krankheitsgrad fokussieren. Dies könnte zu einem stärkeren Krankheitserleben und einem intensiveren Vermeidungsverhalten führen. "Radiography of the lumbar spine in primary care patients with low back pain of at least six weeks' duration is not associated with improved patient functioning, severity of pain, or overall health status but is associated with an increase in doctor workload." (S. 400)

\section{Merkmale der Behandlung}

- $\quad$ Ungenügende Patientenedukation und -motivation

Einen wesentlichen Bestandteil der Behandlung von Rückenschmerzpatienten stellt die Wissensvermittlung und Informationsweitergabe hinsichtlich Entstehung und Verlauf des persönlichen Krankheitsbildes dar. Hierunter versteht man ein geordnetes, didaktisch dem Bildungsniveau des Patienten angemessenes Gespräch, welches zwar ausführlich, aber nicht zu detailliert über das jeweilige Krankheitsbild informiert. Der 
Patient sollte erfahren, um welche Art von Erkrankung es sich handelt, einen kurzen Überblick über mögliche Ursachen bekommen, über die häufigsten Behandlungsstrategien aufgeklärt werden und realistische Aussagen bezüglich der Genesung erhalten. Jedoch sollte der Patient zu Beginn der Diagnosestellung nicht mit kompliziertem Detailwissen überfordert und verwirrt werden. Ggf. kann auch der Einsatz von Vorträgen, Broschüren oder Informationsfilmen hilfreich sein.

In der Information sollte i.d.R. die vorzufindende Harmlosigkeit von Rückenbeschwerden verdeutlicht werden. Den Patienten sollte z.B. auch erklärt werden, dass ca. 85\% der Bevölkerung westlicher Industriestaaten mindestens einmal in ihrem Leben an Rückenbeschwerden leiden (Hildebrandt, Mense 2001) und dass 68\% aller Patienten, die innerhalb einer Woche ihren Allgemeinmediziner konsultieren, Rückenschmerzpatienten sind (in DEGAM-Leitlinie Nr. 3: Kreuzschmerzen 2003). Dies soll bei den Patienten keinesfalls den Eindruck erwecken, dass sie aufgrund der hohen Patientenzahlen nicht mehr ernst genug genommen werden. Es soll ihnen vielmehr zeigen, dass ihr betreuender Arzt sehr häufig mit diesem Krankheitsbild konfrontiert wird, dadurch eine gewisse Sicherheit und Routine in der Diagnosestellung und Behandlung besitzt und vor allem, dass sie mit ihren Beschwerden kein Einzelfall sind. Auch die Ärzte könnten durch eine gewissenhaftere Anamnese für sich selbst eine bessere Zuordnung der Patienten in wahrscheinlich eher unkomplizierte Beschwerden versus Risikopatient schaffen, indem sie z.B. die yellow flags verstärkt erfragen und den Umfang der körperlichen Untersuchung erweitern.

Nur ungefähr die Hälfte unserer befragten Patienten (52\%) gibt an, ihrer Meinung nach adäquat über ihr Krankheitsbild (inkl. Ursachen, Behandlungsmöglichkeiten, Prognose) aufgeklärt worden zu sein. Wenn überhaupt, dann soll ein solches Aufklärungsgespräch meist erst im fortgeschrittenen Verlauf der Erkrankung stattgefunden haben. Ungefähr 2/3 gaben an, dass sie erst während einer Rehabilitationsmaßnahme ein ausführliches Informationsgespräch erhielten.

Dass nur ungefähr die Hälfte aller Befragten ihrer Meinung nach angemessen über ihr Krankheitsbild informiert wurde, und meist erst im fortgeschrittenen Verlauf der Erkrankung, lässt den Schluss zu, dass die Patientenedukation deutlich intensiviert werden muss. Die Information sollte angepasst sein an das Bildungsniveau und das 
Alter der Patienten und entsprechende Beeinträchtigungen (Sprachverständnis) berücksichtigen (s.o.).

Sofern noch nicht geschehen, sollte dies dringend vom betreuenden Hausarzt, (der oft den zentralen Ansprechpartner für unsere befragten Rückenschmerzpatienten darstellte), nachgeholt werden.

Außerdem sollte diese wichtige Patienten-Information später nochmals in den jeweiligen multimodalen Schmerzzentren vermittelt werden. Leider finden jedoch, wie bereits erwähnt, viele der Patienten sehr spät oder gar keinen Zugang zu diesen speziellen Schmerzkliniken, so dass eine frühzeitige Mitteilung absolut erstrebenswert ist.

Entsprechend den LL-Empfehlungen wäre außerdem eine Verbesserung der Patientenmotivation in Bezug auf die Ausübung körperlicher Aktivitäten wünschenswert. Immerhin 3/4 aller Patienten soll nach ihrer Erinnerung gesondert darauf hingewiesen worden sein, dass Bewegung nicht schadet, sondern dadurch ihre Genesung gefördert werden würde. Dies ist prinzipiell ein erfreulicher Wert, wenn auch noch verbesserungsfähig. Empfehlungen sind dann erfolgreicher für eine Umsetzung, wenn sie möglichst konkret ausfallen und nicht nur pauschal.

28\% der befragten Patienten erinnern sich daran, von ihren Ärzten konkrete Empfehlungen und Informationen zu bestehenden Sportprogrammen in der Umgebung erhalten zu haben. Entsprechend war dies bei 72\% nicht der Fall. Es wäre in diesem Zusammenhang sinnvoll, wenn die Behandler über lokale Sport- und Fitnessangebote ausreichend informiert sind, und diese Information in Form von Broschüren weitergeben könnten. Bzgl. dieses Punktes (z.B. Zusammenstellung der Informationen) könnten auch öffentliche Einrichtungen oder Krankenkassen mehr Aufgaben übernehmen.

In der Literatur werden durchaus positive Erfahrungen mit der Effektivität von Aufklärungskampagnen berichtet: Eine australische Studie (Buchbinder et al. 2001) untersuchte den Effekt einer Medienkampagne, die Aufklärung und verstärkte Informationsweitergabe bezüglich Kreuzschmerzen zum Ziel hatte. Es wurde geprüft, ob die Medienkampagne Auswirkungen auf die persönliche Einstellung der Bevölkerung zum Thema Rückenschmerzen sowie ihr Denken und Handeln hatte und ob daraus Veränderungen bezüglich Management und Umgang mit Kreuzschmerzen resultierten. Das Ergebnis war, dass die öffentliche Informationsweitergabe zu einer deutlichen 
Verbesserung des Denken und Handelns der Allgemeinbevölkerung in Bezug auf ihren Umgang bzw. ihre Ängste mit Rückenschmerzen führte. Es veränderten sich sowohl die sog. „fear avoidance beliefs" (Verringerung) als auch die physischen Aktivitäten der Bevölkerung (Anstieg) (Buchbinder et al. 2001, S. 2535). In einer späteren Studie in England (Burton et al. 1999) konnten diese überaus positiven Ergebnisse zwar pauschal nicht repliziert werden, doch bestätigte sich, dass Patienten mit initial höheren Fear-avoidance-beliefs bzw. mit größerem Angst-Vermeidungs-Verhalten nach Aufklärungs-Maßnahmen stärkere positive Veränderungen ihrer Einstellungen zu Rückenschmerzen erlebten.

Darlow et al. (2012) beschreiben außerdem, dass sich Ärzte bzw. medizinisches Personal intensiv über die Verbindung von ihren Ansichten und Haltungen, mit denen der Patienten bewusst sein müssen (S. 3). Anhand ihrer Studie konnten sie feststellen, dass Ärzte, die selbst über verstärkte fear-avoidance-beliefs verfügen, ihre Patienten auch eher zu Ruhe und eingeschränkten Bewegungen motivieren und eben nicht zu dosierter Aktivität. Weiterhin zeigte sich, dass Ärzte mit erhöhten eigenen AngstVermeidungs-Überzeugungen auch zu einem vermehrten bzw. schnelleren Ausstellen von Krankschreibungen tendieren (S. 3).

Abschließend ist zu sagen, dass eine adäquate Informationsweitergabe absolut unerlässlich ist: sie muss zielgerichtet sein und gleichzeitig dem Patienten die nötige Beruhigung vermitteln. Um das zu erreichen, ist nicht nur Zeit und Engagement wichtig, sondern auch die nötige Unterstützung z.B. von Seiten der Krankenkassen, die sich z.B. mit geeignetem Informationsmaterial beteiligen könnten. Die Information darf nicht zu einer (unterfinanzierten) Belastung für die Ärzte werden.

- $\quad$ Schmerzmittel als Erst- und Haupttherapeutikum, NSAR im Mittelpunkt der Schmerzmedikation

73\% aller Patienten berichten darüber, als Ersttherapeutikum ein Schmerzmittel verschrieben bekommen zu haben. Dabei stellten die Nichtsteroidalen Antirheumatika die am häufigsten verwendete Wirkstoffklasse der Schmerzmedikation dar: Ibuprofen (Einnahmehäufigkeit 74\%), Diclofenac (Voltaren) (46\%). Bei beiden Medikamenten 
muss bedacht werden, dass sie in geringer Dosierung frei verkäuflich sind, ihr Konsum also nicht zwingend von der ärztlichen Verschreibung abhängt.

Weiterhin wurden Tilidin (Valoron) und Metamizol (Novalgin) von fast der Hälfte unserer Befragten über einen regelmäßigen Zeitraum (mindestens 3 Monate) eingenommen. Außerdem bedeutsam waren Tramadol, Pregabalin (Lyrika) sowie Coxibe. Auch trizyklische Antidepressiva waren häufiger vertreten.

Insgesamt nahmen die Patienten für ihren Rückenschmerz im Verlauf ihrer Erkrankung durchschnittlich 4 verschreibungspflichtige Medikamente regelmäßig ein. Die Spannweite betrug dabei von 1 bis zu 8 Präparaten (SD 1,94).

Leider konnten die Patienten aufgrund der oft umfangreichen Patientenkarrieren keine näheren und zuverlässigen Angaben zur Dauer des jeweiligen Schmerzmittelgebrauchs machen.

Generell besagen die Leitlinien, dass die medikamentöse Therapie vor allem als Unterstützung dafür dienen soll, um dem Patienten therapeutische Maßnahmen sowie Alltagsaktivitäten zu ermöglichen. Deshalb sollen nicht notwendige Medikationen und unnötig hohe Konzentrationen vermieden werden. Dennoch bekamen laut unserer Befragung 3/4 (73\%) aller Patienten ein Schmerzmittel als alleiniges Erst- und Haupttherapeutikum. Auch die durchschnittliche Anzahl von 4 verschreibungspflichtigen Schmerzmedikamenten pro Patient ist überdenkenswert.

Nichtsteroidale Antirheumatika stehen offensichtlich im Mittelpunkt der Schmerzmedikation. Diese können laut der "Nationalen Versorgungsleitlinie Kreuzschmerz" (Version 1.2., 2011) zwar sowohl zur Behandlung des akuten nichtspezifischen Kreuzschmerzes als auch des chronischen nicht-spezifischen Rückenschmerzes verwendet werden, sollen jedoch nur "für kurze Zeit" und „in geringer Dosis“ („Nationalen Versorgungsleitlinie Kreuzschmerz“, Version 1.2., 2011, S. 31) verabreicht werden. In unserer Stichprobe fanden Ibuprofen und Diclofenac jedoch meistens als Dauermedikation Anwendung.

Ibuprofen und Diclofenac sind leicht zugängliche Medikamente und in einem Großteil der deutschen Haushalte problemlos verfügbar. Aufgrund der verbreiteten Meinung, dass (freiverkäufliche) Medikamente wenig Schaden anrichten können, finden sie oft Anwendung. Ibuprofen in der Dosierung von 400mg ist beispielsweise rezeptfrei 
erhältlich. Ob sich Patienten an diese geringe Dosierungsempfehlung halten, ist allerdings fraglich.

Einserseits ist es richtig, dass die NSAR im Rahmen des Gebrauchs von gesunden Patienten relativ ungefährlich sind, dennoch darf nicht vergessen werden, dass etwaige UAW wie Reizungen der Schleimhaut des Gastrointestinaltrakts bei bestimmten Risikogruppen, wie z.B. Patienten mit Ulcera-Anamnese, schwerwiegende Folgen haben können.

Die Annahme, dass NSAR oftmals weniger schwerwiegende Nebenwirkungen als z.B. niedrigdosierte Opioide oder Metamizol hervorrufen sowie die leichte Zugänglichkeit der Medikamente, führt möglicherweise zu der hohen Anzahl an Patienten, die mit Ibuprofen oder Diclofenac über einen längerfristigen Zeitraum behandelt wurden.

Führt die Behandlung mit Nicht-Opioiden nicht zum Erfolg, so können beim nichtspezifischen Kreuzschmerz schwache Opioide zum Einsatz kommen. Hierzu zählen z.B. Tramadol und Tilidin/ Naloxon. Tilidin war das Medikament, welches von unseren Patienten am 3. häufigsten (45\% der Befragten) über längere Zeit eingenommen wurde. Obwohl die Einnahme von Pregabalin (Lyrika) laut Leitlinie nicht empfohlen wird, fand dieses Medikament bei immerhin ca. 1/4 der Patienten Anwendung.

Dies könnte möglicherweise daran liegen, dass Lyrika aus der Gruppe der Antikonvulsiva z.B. gute Wirksamkeit bei neuropathischen Schmerzen zeigt und möglicherweise deswegen einige Ärzte der Meinung sind, dass nicht-spezifische Rückenschmerz-Patienten auch davon profitieren können.

Auch Cox 2-Hemmer sollen laut der „Nationalen Versorgungsleitlinie Kreuzschmerz" (Version 1.2., 2011) sowohl beim akuten als auch beim chronischen nicht-spezifischen Kreuzschmerz nur bei NSAR-Unverträglichkeit verordnet werden und gelten heute als off label use. Dennoch ist die Anzahl der Patienten, die nach ihrer Erinnerung mit Coxiben behandelt wurden, als relativ hoch einzuordnen (24\%).

Seit dem Jahr 1999 wurden in Deutschland verschiedene Coxibe zugelassen (z.B. Rofecoxib, Valdecoxib, Etoricoxib, Lumiracoxib, Celecoxib) jedoch auf Grund ihres vor allem kardiovaskulären Risikos teilweise wieder vom Markt genommen. Eine offizielle 
Zulassung zur Behandlung von Rückenschmerzen bestand für keines der Präparate. Lediglich für Rofecoxib (zugelassen 1999 bis 2004) bestand die Zulassung zur „Linderung akuter Schmerzen“. Ansonsten war und ist die Zulassung vor allem auf entzündliche und degenerative Gelenkerkrankungen beschränkt (Celecoxib, Valdecoxib, Etoricoxib). Eine Off- Label Anwendung wird jedoch in vielen Veröffentlichungen nach wie vor diskutiert und zum Teil auch empfohlen. (Bundesinstitut für Arzneimittel und Medizinprodukte, 2005).

Zusammengefasst stehen beim nicht-spezifischen Rückenschmerz die NSAR im Mittelpunkt der Schmerzmedikation, deren Nebenwirkungsprofil trotz der offensichtlichen „Ungefährlichkeit“ genauestens überwacht werden sollte. Weiterhin sollte versucht werden, die Medikation dem WHO-Stufenschema gemäß anzupassen (s. Abb. 19), bei dem zuerst ein Nicht-Opioid-Analgetika zum Einsatz kommen soll, gefolgt von schwachen Opioiden (nötigenfalls kombiniert mit Nicht-Opioid-Analgetika). Erst wenn weder Nicht-Opioid-Analgetika noch schwache Opioide den gewünschten Effekt zeigen, sollen starke Opioide eingesetzt werden. Co-Analgetika, wie z.B. Antidepressiva, sind laut WHO in allen Phasen indiziert.

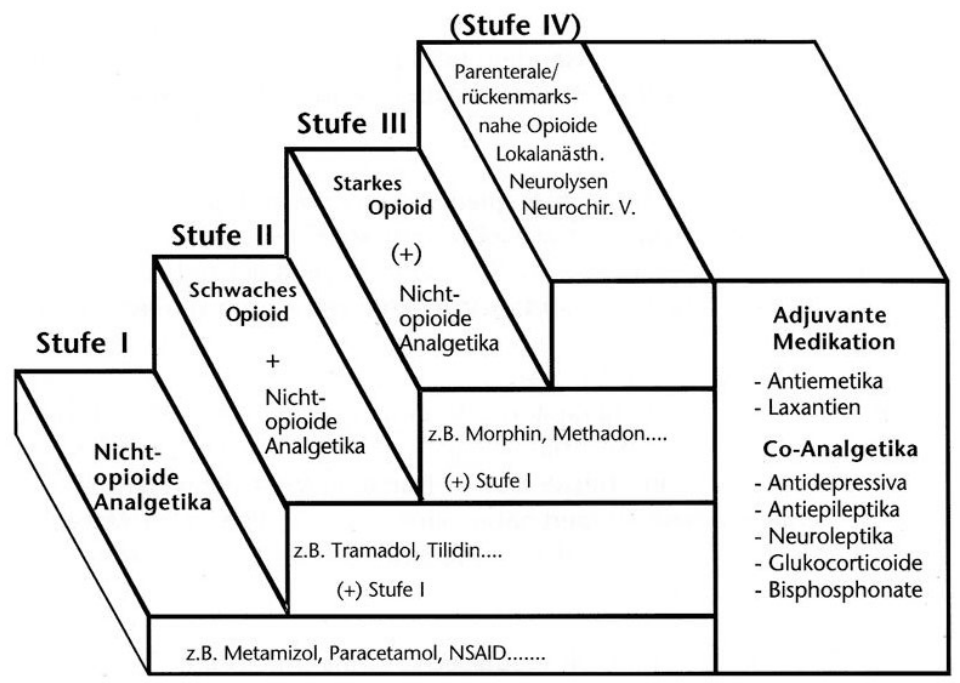

Abb. 19

WHO-Stufenschema der medikamentösen Schmerztherapie (http://afag-carre.de/index.php?page=medikamentoese-schmerztherapie (2013) 
- $\quad$ Ungenügende Medikamenten-Aufklärung, fehlendes festes Zeitschema

Im Falle der fest verordneten Medikamente scheinen fast alle Patienten (96\%) erfreulicherweise, über die Dosierung informiert worden zu sein. Problematisch waren allerdings die Erläuterungen zur Einnahmedauer: nur knapp 1/4 aller Probanden gaben an, explizit darauf hingewiesen worden zu sein, wie lange sie das gewählte Präparat einnehmen können/ sollen. Oft wurde der Begriff bei Bedarf verwendet, eine fest angesetzte Einnahmedauer und -häufigkeit gab es nicht.

Diese Einnahmeanweisung steht im Widerspruch zu der in der Schmerztherapie bevorzugten Strategie der nicht schmerz-kontingent sondern zeit-kontingent eingenommenen Einnahme. Hierbei sollen durch eine regelmäßige Einnahme der Medikamente die Schmerzspitzen verhindert und eine kontinuierliche Analgesie garantiert werden. Leider greifen viele Patienten dennoch nur dann zu Schmerzmitteln, sobald sie den Schmerz bzw. eine Schmerzverstärkung verspüren, (dann jedoch häufig auch zusätzlich in höherer Dosierung als eigentlich notwendig gewesen wäre, wenn sie die Medikamente zeit-kontingent/ zu fest vorgeschrieben Zeiten trotz Schmerzfreiheit eingenommen hätten).

Auch die Aufklärung der Patienten über mögliche Nebenwirkungen scheint als unzureichend einzustufen zu sein: nur 18\% wurden laut ihrer Aussage über etwaige unerwünschte Arzneimittelwirkungen aufgeklärt, obwohl später in $84 \%$ der Fälle UAWs auftraten.

Es stellt sich die Frage, warum die Ärzte oft nicht über die häufig vorkommenden Nebenwirkungen im Voraus berichten. Ein Grund ist sicherlich, dass sie nicht unnötige Verunsicherung beim Patienten hervorrufen wollen und die Sorge, dass Patienten die Medikamente ablehnen könnten. Andererseits würden die Patienten im Falle des Auftretens von UAW weniger ängstlich sein, wenn sie im Voraus darauf vorbereitet worden wären. Ein weiterer Grund kann auch wie bereits mehrfach erwähnt schlichtweg der Zeitfaktor sein, der die aufklärenden Gespräche limitiert.

Zusammenfassend kann die Informationsweitergabe bezüglich der Schmerzmitteldosierung als Fazit zufriedenstellend eingeordnet werden. Die Informationsweitergabe bezüglich der Einnahmedauer und Einnahmehäufigkeit ist 
möglicherweise aber verbesserungspflichtig. Die Formulierung bei Bedarf sollte grundsätzlich vermieden werden. Laut Leitlinie ist es wichtig, dass die Medikamenteneinnahme einem festen Schema folgt.

- $\quad$ Fast routinemäßige Physiotherapie-Verordnung

Betrachtet man die Zahlen der Krankengymnastik-Verordnungen, so fällt auf, dass fast jeder der befragten Patienten (92\%) angab, in den letzten 3 Jahren mit rückenspezifischer Physiotherapie behandelt worden zu sein. Die Gesamtmenge der Verschreibungen betrug bezogen auf die letzten 3 Jahre durchschnittlich 7 Rezepte à 6 Termine (42 Behandlungen).

Diese Menge an Krankengymnastik-Verordnungen steht in deutlichem Widerspruch sowohl $\mathrm{zu}$ den Leitlinienempfehlungen der Nationalen Versorgungsleitlinie Kreuzschmerz als auch den Heilmittelrichtlinien (nach Paragraph 92 SGB V). Laut Heilmittelkatalog sind für den chronischen nicht-spezifischen Kreuzschmerz durchschnittlich 18 Behandlungen (3 mal 6) als Gesamtverordnungsmenge angedacht. Demnach erscheinen durchschnittlich 42 Behandlungen pro Patient als deutlich zu viel. Auch Bekkering et al. (2003) berichteten von einer ungerechtfertigt hohen Verschreibungsmenge von Krankengymnastik. Grundsätzlich soll laut Leitlinie beim nicht-spezifischen Kreuzschmerz die Aktivierung der Patienten zwar im Vordergrund stehen, jedoch in Form von kontrollierter Bewegungstherapie mit verhaltenstherapeutischem Ansatz. Fast alle physiotherapeutischen passiven Behandlungsmethoden werden dort als nicht wirksam beschrieben. Diese Fehl- bzw. Überversorgung wird laut Arnold et al. 2011 außerdem anhand des GEK-Heil- und Hilfsmittel-Reports 2006 deutlich, der über eine Krankengymnastik-Verordung in $50 \%$ der Fälle aller nichtspezifischen Rückenschmerzen berichtet, obwohl diese nicht in den Leitlinien empfohlen wird. Manuelle Therapie (laut Leitlinien empfohlen) soll dagegen laut Bericht nur in ca. 12-13\% der Fälle verordnet worden sein (Arnold et al. 2011). Erschwerend zu diesen offensichtlichen physiotherapeutischen Fehl-, Unter- und Überverordnungen kommt hinzu, dass laut Angaben der Patienten ein Teil der spezifisch verordneten Physiotherapie (meist auf eigenen Wunsch der Patienten hin) in Massagen umgewandelt wird. Dies geschieht offensichtlich in Absprache mit den 
Physiotherapie-Praxen und entgegen der ärztlichen Verordnung. Dadurch wird dem eigentlichen gewünschten Effekt, die Selbstwirksamkeit der Patienten zu fördern, nicht entsprochen und eine Maßnahme durchgeführt, die der generellen Tendenz der NVL, passive therapeutische Maßnahmen nicht zu empfehlen, widerspricht. Grundsätzlich soll laut Leitlinie beim nicht-spezifischen Kreuzschmerz die Aktivierung der Patienten im Vordergrund stehen.

Wichtig ist, dass akuter nicht-spezifischer Kreuzschmerz keinesfalls mit Bettruhe behandelt werden soll. Eine zügige Rückkehr zu den gewohnten Aktivitäten wird angestrebt. Auch bei chronischem Rückenschmerz wird von Bettruhe abgeraten, da diese die Rehabilitation erschwert und das "passive Krankheitsverhalten“ ("Nationalen Versorgungsleitlinie Kreuzschmerz", Version 1.2., 2011, S. 23) verstärkt. Generell sollten die Patienten also zu körperlicher Aktivität motiviert werden. Diese muss nicht (ausschließlich) über Krankengymnastik erfolgen, sondern soll unabhängig von medizinischen Maßnahmen entstehen. Krankengymnastik kann diesen Prozess der Aktivierung unterstützen, wobei nicht die eigentliche krankengymnastische Technik zur Behandlung von individuellen Defiziten gefragt ist, sondern die physiotherapeutische Expertise und Beratung in Fragen der generellen körperlichen Aktivierung.

Wie bereits erwähnt, wird oft ein Teil der Physiotherapie Verordnungen in Massagen umgewandelt. Da auch Massagen die Passivität des Patienten unterstützen bzw. ihn möglicherweise nicht zu ausreichender Eigenaktivität motivieren, wird diese Behandlungsform für akute Kreuzschmerz-Patienten nicht empfohlen. Hier gälte es entsprechend Maßnahmen zu ergreifen, dass eine solche Umwandlung nicht vorgenommen werden kann.

\section{- $\quad$ Häufige Anwendung invasiver Verfahren}

Hierbei beziehen wir uns besonders auf Injektionen in den Rücken (mit und ohne Bildgebung). 34\% unserer Befragten gaben an, schon bei der ersten Arztkonsultation mit Injektionen in den Rücken behandelt worden zu sein. $76 \%$ haben im Laufe ihrer Behandlung mindestens einmal Injektionen in den Rücken (auch ohne Bildgebung) erhalten. 
Laut der „Nationalen Versorgungsleitlinie Kreuzschmerz" (Version 1.2., 2011) sollen bei nicht-spezifischen Rückenschmerzen aufgrund fehlender Effektivitäts-Studien generell keine invasiven Behandlungsverfahren angewendet werden. Wir konzentrierten uns in der Befragung besonders auf Injektionen in den Rücken (mit und ohne Bildgebung). Nahezu 1/3 der Patienten wollen sich daran erinnern, dass sie bereits beim Erstkontakt eine Injektion erhalten haben. Abgesehen davon, dass es keine Evidenz für die Wirksamkeit von Injektionsbehandlungen beim nicht-spezifischen Schmerz gibt, verstärken diese Maßnahmen das passive Krankheitsmodell der Patienten.

Leider wird oft zu schnell zu invasiven Methoden gegriffen. Dies mag einerseits an den Patientenerwartungen liegen, schneller Schmerzfreiheit zu erreichen, andererseits spielen sicherlich auch finanzielle Anreize auf Seiten der Ärzte eine Rolle.

Leider vergessen viele Patienten, dass zum einen durch die invasiven Maßnahmen oder Operationen oftmals nicht die Ursache der Beschwerden therapiert wird und andererseits dadurch auch bestimmte Risiken, wie z.B. die Gefahr von Infektionen bestehen.

Diese Variante schneller und bequemer vorrübergehende Schmerzfreiheit zu erreichen, ist leider oft sehr verlockend im Vergleich zum teilweise sehr mühsamen Weg der nichtinvasiven Therapien.

\section{- $\quad$ Zu späte Verordnung von multimodaler Therapie}

Die Schmerzdauer unserer Patienten lag zum Zeitpunkt der Befragung bei einigen Befragten bei bereits 30 Jahren (s.o.). Mehr als die Hälfte (56\%) hatte seit mindestens 10 Jahren Beschwerden. Die „Nationale Versorgungsleitlinie Kreuzschmerz" sieht bereits ab 12 Wochen Krankheitsdauer die Überprüfung der Notwendigkeit multimodaler, interdisziplinärer Therapie vor. Eine solche Prüfung hat laut unserer Befragten jedoch in den wenigsten Fällen stattgefunden; die Patienten wurden ihren Angaben zufolge jahrelang auf immer die gleiche Weise behandelt, obwohl es letztlich zu keiner durchschlagenen Veränderung kam. So sollen viele von ihnen jahrelang mit Massagen oder Krankengymnastik behandelt worden sein. Für nahezu jeden unserer Studienteilnehmer stellte das Göttinger Tagesklinik Programm die erste Berührung mit 
multimodaler (gleichzeitig interdiszipinärer) Therapie dar. Laut der „Nationalen Versorgungsleitlinie Kreuzschmerz" (Version 1.2., 2011) sollen Patienten mit nichtspezifischen Kreuzschmerzen - insbesondere wenn sich psychosoziale Risikofaktoren (sog. yellow flags) identifizieren lassen - so früh wie möglich in ein multimodales und interdisziplinäres Behandlungsprogramm einbezogen werden. Bei Beschwerden, die trotz leitlinien-gerechter Therapie über 12 Wochen anhalten, sollte die Teilnahme an einem multimodalen Programm generell in Betracht gezogen werden. Hierunter versteht man eine Therapieform, in die mindestens 3 verschiedene Berufsgruppen in ein interdisziplinäres Konzept einbezogen sind (Mediziner/ Schmerztherapeuten, Psychologen/ Psychotherapeuten, Physiotherapeuten, Ergotherapeuten). Eine solche Vorgehensweise ist sicherlich sinnvoll, andererseits muss aber auch die Umsetzbarkeit überprüft werden. Es ist z.B. kritisch zu hinterfragen, ob in der jeweiligen Region ausreichende diagnostische Kapazitäten und entsprechende Expertise bereitgehalten werden, um diese Prüfung vornehmen zu können. Wenn man davon ausgeht, dass It. epidemiologischen Annahmen ca. 10-15\% aller Patienten mit Kreuzschmerzen ein Chronifizierungsrisiko aufweisen (Fahland et al. 2011), wäre dies in Anbetracht der hohen Prävalenzzahlen dieses Krankheitsbildes eine so große Gruppe, dass die derzeit vorhandenen Kapazitäten interdisziplinärer Diagnostik dafür sicher nicht ausreichen würden. Hier sind in letzter Zeit interessante Anstrengungen erkennbar, diese Diagnostik über ein kurzes Screening-Instrument zu erleichtern (Schmidt et al. 2010). Angesichts der regional sehr unterschiedlichen Kapazitäten betrifft eine andere Frage außerdem die Umsetzbarkeit bzw. die zeitgerechte Durchführung multimodaler Therapie. Die multimodale Behandlungsform ist relativ neu und in den Versorgungsalltag in Deutschland bisher nicht regelhaft integriert. Momentan wird sie vor allem an (wenigen) spezialisierten schmerztherapeutischen Einrichtungen angeboten. Ihre Umsetzung ist aufwändig, kostenintensiv und an eine hohe Expertise des interdisziplinären Personals gebunden (Arnold et al. 2009).

Sicherlich wären hierfür eine intensivere Kommunikation untereinander und eine vermehrte Teilnahme an Schmerzkonferenzen förderlich, dennoch muss bedacht werden, dass angesichts des breiten Spektrums an Erkrankungen, das Hausärzte zu versorgen haben, und aufgrund der deutlichen Zunahme des 
Dokumentationsaufwandes es nicht leistbar ist, an immer neuen Fort- und Weiterbildungsveranstaltungen teilzunehmen. Diese gut gedachte Qualifizierung ist in der Versorgungsrealität nicht umsetzbar.

\section{Weitere Ergebnisse:}

- Unsere Patienten unterzogen sich ihrer Erinnerung nach einer Vielzahl ganz unterschiedlichster Behandlungsverfahren: Schmerzmitteleinnahme, Physiotherapie, Injektionen in den Rücken oder ins Gesäß, Rehabilitationsmaßnahmen,Manipulationen, Reizstrom, TENS (= transkutane elektrische Nervenstimulation), Psychotherapie sowie Rückenschulen.

Wie bereits im Kapitel 2.5.2.1 beschrieben, existiert eine Vielzahl unterschiedlicher Varianten an Rückenschulen. Laut Hildebrandt, Pfingsten (2011) sind in der Primärprävention jedoch keine positiven Effekte/ die Wirksamkeit von Rückenschulen belegt worden. Dennoch hat sie unserer Meinung nach einen berechtigten Stellenwert in der Tertiärprävention, bei der die negativen Folgen der bereits bestehenden Beschwerden reduziert werden sollen, um möglichen Komplikationen entgegen zu wirken.

Die hohe Anzahl diverser Therapieangebote zeigt laut Haldeman \& Dagenais (2008) jedoch, dass eine optimale Behandlungsstrategie bisher fehlt. Hier liegt laut Chenot et al. (2009 a) einer der Gründe dafür, warum die Patienten so häufig zu Selbstzahlerleistungen bereit sind. Kauf einer neuen Matratze, Akupunktur, Entspannungsverfahren (Yoga, Pilates, Qi Gong etc.), Massagen, OsteopathieBehandlungen. Hierin spiegelt sich offensichtlich auch eine Unzufriedenheit mit dem durch die übliche Vorgehensweise erzielten Erfolg wieder sowie eine Hilflosigkeit auf Seiten der Patienten, die letztlich „zu jedem Strohhalm“ greifen. Der Kauf von Sportgeräten sowie Aufwendungen für den Beginn neuer Sportarten sind dabei noch sinnvoll anzusehen, weil sie der Zielrichtung der Leitlinie folgen; die passiven Maßnahmen sind jedoch ein Problem.

Insofern ist der Rückenschmerz auch ein lukratives Geschäft geworden, was die Strukturierung einer sinnvollen Vorgehensweise und auch die Einflussmöglichkeiten 
noch erschweren dürfte. Interessant bei den Selbstzahlerleistungen ist die hohe Anzahl der Patienten (42\%), die an einer Akupunktur- Behandlung teilgenommen haben. Zum Zeitpunkt der Datenerhebung waren Akupunktur- Behandlungen noch Privatleistung. Mittlerweile (2013) werden sie teilweise von den gesetzlichen Krankenkassen bewilligt. Da bei dieser Art der Behandlung keine Aktivierung des Patienten erfolgt, wird eine Akupunkturbehandlung bei akutem Rückenschmerz laut Leitlinie jedoch nicht empfohlen; auch bei chronischem Kreuzschmerz werden Akupunktur-Anwendungen kritisiert. Es bleibt aber unklar, ob die Motivation für diese Maßnahme von Seiten der Patienten selbst ausging, oder ob sie im Rahmen der individuellen Gesundheitsleistungen von den Ärzten selbst angeboten wurden. In der Studie von Chenot et al. (2007) ist von einem bereits durch verschiedene Untersuchungen beschriebenen positiven Effekt von Akupunktur auf den Krankheitsverlauf die Rede. Allerdings wird auch beschrieben, dass die häufige Inanspruchnahme von Akupunktur zu keiner, wie zuerst angenommen, Verringerung der Inanspruchnahme anderer alternativer Heil-Verfahren geführt hat.

\subsection{Beeinträchtigungen der Interpretierbarkeit}

Wir haben in unserer Befragung substantielle Ergebnisse bzgl. Mängel in der Versorgung von Patienten mit Rückenscherzen aufzeigen können. Diese Ergebnisse sind z.T. erwartet worden, z.T. waren sie schon in anderen Studien bereits bestätigt (Schulte et al. 2009) worden. Trotz der damit erreichten Validität müssen für unsere Ergebnisse jedoch auch deutliche Vorbehalte berücksichtigt werden. Die Generalisierbarkeit der Resultate wird z.T. erheblich durch die Art der Datenermittlung (Befragung der betroffenen Patienten) eingeschränkt, da sie aufgrund der z.T. langen Krankheitsgeschichte unkalkulierbaren Erinnerungseffekten unterlag. Es handelt sich darüber hinaus um ein relativ kleines Patientenkollektiv, dessen Angaben fast ausschließlich die subjektiven Erfahrungen des Einzelnen wiedergeben.

Wie bereits erwähnt, handelt es sich jedoch keinesfalls um ausgesuchte Patienten, sondern um eine konsekutive Stichprobe einer universitäten schmerztherapeutischen Einrichtung. 
Da der Krankheitsbeginn bei den meisten Patienten oft schon viele Jahre zurücklag, (56\% hatte zum Zeitpunkt der Befragung seit mindestens 10 Jahren Beschwerden), konnten teilweise keine expliziten Details mehr zum Zeitpunkt des erstmaligen Auftretens der Beschwerden erfragt werden. Die Zuverlässigkeit dieser Patientenaussagen muss demnach deutlich als eingeschränkt betrachtet werden. Eine Erklärung für die geringe Berücksichtigung von psychosozialen Risikofaktoren - wie sie sich in der Befragung der Patienten widerspiegelt - könnte darin liegen, dass der oftmals bereits seit vielen Jahren betreuende Hausarzt auf die spezifische Erfragung der yellow flags verzichtet hat, weil er die Umstände des Patienten und seiner Familie bereits kannte.

Ideal wäre es gewesen, wenn wir nur Patienten in die Studie einbezogen hätten, deren Anamnese maximal die letzten 3 Jahre umfasst hätte. Leider war dies jedoch nicht möglich, da ansonsten während der 1-jährigen Laufzeit unserer Studie nur insgesamt 14 der befragten Patienten in die Studie eingeschlossen hätten werden können. Darin zeigt sich andererseits auch wiederum ein Problem in der Versorgungspraxis dieser Patienten: Ihre Überweisung in eine spezialisierte schmerztherapeutische Einrichtung passiert zu spät. Um Patienten früher in ihrer Krankheitsgeschichte zu erfassen, wäre ggf. eine Befragung in allgemeinärztlichen Praxen der bessere Weg gewesen.

Es wirkte sich bei der Befragung überaus erschwerend und zeitraubend aus, dass den Patienten vor Beantwortung des Fragenkataloges bzgl. mehrerer Sachverhalte, deren Kenntnis ursprünglich als bekannt vorausgesetzt wurde, zunächst ausführlichere Erklärungen gegeben werden mussten. So konnten viele Patienten, z.B. erst nach detaillierten Erklärungen der verschiedenen Möglichkeiten radiologischer Bildgebung, eine korrekte Zuordnung dahingehend treffen, ob Sie ein CT oder ein MRT erhalten hatten. Dies verdeutlicht abermals die Dringlichkeit einer besseren Aufklärung der Patienten. Rund die Hälfte der Patienten (53,6\%) wechselte außerdem mindestens einmal innerhalb einer Facharzt-Richtung den Arzt; die meisten sogar mehrfach. Somit muss davon ausgegangen werden, dass es teilweise falsche Zuordnungen der Maßnahmen zum jeweiligen Arzt gegeben haben könnte, schlichtweg weil die große 
Anzahl involvierter Ärzte in der häufig langjährigen Behandlungsgeschichte die Patienten den Überblick verlieren ließ.

Schwierig gestaltete sich außerdem die Unterscheidung der Berufsgruppen Neurologie und Neurochirurgie. Vielen Patienten war bis zu dem von uns durchgeführten Interview kein Unterschied zwischen diesen beiden Facharzt-Richtungen bekannt, so dass auch die Interpretierbarkeit beispielsweise dieser Ergebnisse deutlich eingeschränkt ist. Auch die Differenzierung zwischen Anschlussheilbehandlung, stationärem Aufenthalt und Rehabilitationsaufenthalt stellte sich kompliziert dar.

Retrospektiv wäre eine konkrete Befragung der Patienten zur Zufriedenheit mit ihrem jeweiligen Hausarzt, die genauere Erfragung der Zufriedenheit der Patienten mit ihren Fachärzten bezüglich der durchgeführten diagnostischen und therapeutischen Maßnahmen sowie eine deutlichere Definition der Geschlechterrolle wünschenswert gewesen.

Vergleicht man nun unsere Ergebnisse mit denen der Berliner Studie (Schulte et al. 2009), siehe Kapitel 2.6. (S. 31), so zeigen sich erwartungsgemäß einige Parallelen und vergleichbare Resultate. Dennoch gab es auch gegenteilige Resultate: So war z.B. für unsere Befragten in fast 2/3 der Fälle der Hausarzt der erste Ansprechpartner, während in der Berliner Studie mehr als die Hälfte aller Befragten als ersten Kontakt bereits einen Spezialisten (meist Orthopäden) wählten. Daher sind auch regionale Versorgungsbesonderheiten, bei der Interpretation der Ergebnisse in Betracht zu ziehen.

Bedacht werden muss weiterhin, dass wir nur Querschnittsdaten ermittelt haben und nicht im Längsschnitt untersuchen konnten, was erheblich zuverlässigere Schlussfolgerungen zulassen würde.

Trotz Abgleich des Fragenkataloges mit bereits durchgeführten Studien (Schulte et al. 2009), trotz sehr hilfreicher Inanspruchnahme entsprechender Expertisen aus der hiesigen Allgemeinmedizin und trotz einer Pilot-Befragung würde deshalb ein weiterer Fragenkatalog sicher anders, kürzer, prägnanter und auch präziser ausfallen. 


\section{Zusammenfassung}

Wir haben bei 50 Patienten mit chronischen Rückenschmerzen aufgrund von (subjektiv erinnerten) Patientenangaben die Behandlungsvorgeschichte erfasst und diese auf ihre Leitlinienkompatibilität geprüft.

Die meisten unserer Ergebnisse zeigen, dass in der Versorgung von Patienten mit Kreuzschmerzen möglicherweise noch ein (mitunter deutlicher) Verbesserungsbedarf besteht.

Insgesamt sollte die körperliche Untersuchung intensiviert werden und den psychosozialen Risikofaktoren mehr Beachtung zukommen. Auch die Indikationen zur Verordnung von Bildgebungen sollten nach den Maßgaben der Leitlinienempfehlungen strenger gestellt werden. Des Weiteren sollte die Unterscheidung von Primär- und Sekundärversorgung stärker gefördert und ein früherer Eintritt in multimodale Therapieprojekte angestrebt werden. Außerdem sind eine verbesserte Patientenedukation und eine stärkere Motivation zu körperlichen Aktivitäten wünschenswert. Nicht notwendige Medikationen sollten vermieden und der häufige Dauereinsatz von Nichtsteroidalen Antirheumatika überdacht werden. Generell ist außerdem eine bessere Aufklärung über mögliche unerwünschte Arzneimittelwirkungen anzustreben. Hierbei sollte u.a. auch auf mögliche Nebenwirkungen bei längerem Gebrauch von Paracetamol verstärkt aufmerksam gemacht werden. Wichtig ist die Einführung fester zeitlicher Schemata bezüglich der Medikamenteneinnahme. Der Begriff „bei Bedarf“ ist teilweise irreführend, wird oft missverstanden und führt dadurch zum problematischen Gebrauch sowie häufig ausbleibendem Behandlungserfolg. Weiterhin sollte der Gebrauch von Physiotherapiebehandlungen, Injektionen in den Rücken sowie Elektrotherapie kritischer überdacht werden.

Insgesamt kann jedoch aufgrund der retrospektiven, methodisch problematischen Betrachtung nicht sicher valide auf „erhebliche Mängel“ in der Versorgung geschlossen werden. 
Trotz allem weist unsere Studie jedoch auch auf einige problematische Aspekte i.S. der Leitlinienkompatibilität hin und regt zu Verbesserungen an.

Bis zur vollständigen Umsetzung der Leitlinien-Empfehlungen ist es sicherlich noch ein weiter Weg. Dieser Weg lohnt sich allemal für die Patienten und für das Gesundheitssystem. Dass er sich auch für Behandler lohnt, wird vermutlich von einer Änderung der Gebührenordnung abhängen, die invasive Therapien und BildgebungsLeistungen weniger honoriert als die sprechende Aktivität des Arztes. 


\section{Abkürzungsverzeichnis}

Abb.: $\quad$ Abbildung

ADS: $\quad$ Allgemeine Depressionsskala

BMI: $\quad$ Body Mass Index

BTM: Betäubungsmittel

BWS: Brustwirbelsäule

bzgl.: bezüglich

bzw.: beziehungsweise

ca.: circa

COX: Cyclooxygenase

CT: $\quad$ Computertomographie

DEGAM: Deutsche Gesellschaft für Allgemeinmedizin und Familienmedizin

DGS: Deutsche Gesellschaft für Schmerztherapie

DGSS: Deutsche Gesellschaft zum Studium des Schmerzes

d.h.: das heißt

DGOOC: Deutsche Gesellschaft für Orthopädie und Orthopädische Chirurgie

DSF: Deutscher Schmerzfragebogen

einschl.: $\quad$ einschließlich

et al.: $\quad$ et alii

etc.: $\quad$ et cetera

evtl.: $\quad$ eventuell

GCPS: $\quad$ Graded chronic pain status

GdB: $\quad$ Grad der Behinderung

ggf.: $\quad$ gegebenenfalls

HADS: Hospital Anxiety and Depression Scale

HWS: Halswirbelsäule

ICD: International Classification of Diseases

i.d.R.: $\quad$ in der Regel

inkl.: $\quad$ inklusive

i.S.: $\quad$ im Sinne 


$\begin{array}{ll}\text { Kap.: } & \text { Kapitel } \\ \text { It:: } & \text { laut } \\ \text { LWS: } & \text { Lendenwirbelsäule } \\ \text { M.: } & \text { Musculus } \\ \text { MdE: } & \text { Minderung der Erwerbsfähigkeit } \\ \text { MPSS: } & \text { Mainzer Pain Staging System } \\ \text { Mrd.: } & \text { Milliarden } \\ \text { MRT: } & \text { Magnetresonanztomographie } \\ \text { NRS: } & \text { Numerische Rating Skala } \\ \text { n.s.: } & \text { nicht signifikant } \\ \text { NSAR: } & \text { Nichtsteroidale Antirheumatika } \\ \text { NVL: } & \text { Nationale Versorgungsleitlinien } \\ \text { O.ä.: } & \text { oder ähnliche } \\ \text { o.J.: } & \text { ohne Jahr } \\ \text { o.O.: } & \text { ohne Ort } \\ \text { O. Verl.: } & \text { ohne Verlag } \\ \text { PDI: } & \text { Pain Disability Index } \\ \text { PENS: } & \text { perkutane elektrische Nervenstimulation } \\ \text { PMR: } & \text { Progressive Muskelrelaxation } \\ \text { S.: } & \text { Seite } \\ \text { S.: } & \text { siehe } \\ \text { SBL: } & \text { Schmerzbeschreibungsliste } \\ \text { SD: } & \text { Standard Deviation (Standartabweichung) } \\ \text { SES: } & \text { Schmerzempfindungsskala } \\ \text { SF-12: } & \text { Short-Form 12 } \\ \text { SGB: } & \text { Sozialgesetzbuch } \\ \text { S.o.: } & \text { siehe oben } \\ \text { sog.: } & \text { sogenannt } \\ \text { SPC: } & \text { specialized pain clinic } \\ \text { SPSS: } & \text { Firma für Analyse- und Statistik-Software }\end{array}$


SSNRI: $\quad$ selektiver Serotonin Noradrenalin Reuptake-Inhibitor (=Wiederaufnahme-Hemmer)

s.u.: $\quad$ siehe unten

Tab.: Tabelle

TENS: $\quad$ Transkutane elektrische Nervenstimulation

u.a.: $\quad$ unter anderem

UAW: unerwünschte Arzneimittelnebenwirkungen

vs.: $\quad$ versus

WHO: World Health Organization, Weltgesundheitsorganisation

z.B.: $\quad$ zum Beispiel

z.T.: $\quad$ zum Teil 


\section{Literaturverzeichnis}

ADS-Handbuch Universität Freiburg: http://www.unifr.ch/ztd/HTS/inftest/WEBInformationssystem/de/4de001/97780fa29a0c11d4b5280000b4909ce2/hb.htm Abrufdatum: 28.10.2012

Arnold B, Böhle E, Chenot JF, Hildebrandt J, Pfingsten M: Versorgungskoordination. In: Hildebrandt J, Pfingsten M (Hrsg.) Rückenschmerz und Lendenwirbelsäule. Elsevier: Urban \& Fischer, München 2011, 453-470

Arnold B, Brinkschmidt T, Casser HR, Gralowl, Irnich D, Klimczyk K, Müller G, Nagel B, Pfingsten M, Schiltenwolf M, Sittl R, Söllner W (2009): Multimodale Schmerztherapie Konzepte und Indikation. Schmerz 23: 112-120

Becker A, Kögel K, Donner-Banzhoff N, Basler H-D, Chenot JF, Maitra R, Kochen MM. (2003): Kreuzschmerzpatienten in der hausärztlichen Praxis: Beschwerden, Behandlungserwartungen und Versorgungsdaten. Z Allg Med 79: 126-131; auch in: DEGAM Leitlinie Nr. 3: Kreuzschmerzen 2003

Becker A, Held H, Redaelli M, Strauch K, Chenot JF, Leonhardt C, Keller S, Baum E, Pfingsten M, Hildebrandt J et al. (2010): Low back pain in primary care: Costs of Care and Prediction of Future Health Care Utilization. Spine $\underline{35}$, Nr 18, 1714-1720

Bekkering GE, Engers AJ, Wensing M, Hendriks HJM, van Tulder MW, Oostendorp RAB, Bouter LM (2003): Development of an implementation strategy for physiotherapy guidelines on low back pain. Aust J Physiother $\underline{49}$, 208-214

Bethge M (2010): Rückenschmerzpatienten. Psychosoziale arbeitsplatzbezogene Faktoren und berufliche Wiedereingliederung - eine Literaturübersicht. Orthopäde 39: 866-873

Buchbinder R, Jolley D, Wyatt M (2001): 2001 Volvo Award Winner in Clinical Studies: Effects of a Media Campaign on Back Pain Beliefs and Its Potential Influence on Management of Low Back Pain in General Practice. Spine 26, Nr. 23, 2535-2542 
Burton AK, Tillotston KM, Main CJ, Hollis S (1995): Psychosocial predictors of outcome in acute and subchronic low back trouble. Spine $\underline{20}, 722-728$

Burton AK, Waddell G, Tillotston KM, Summerton N (1999): Information and advice to patients with back pain can have a positive effect: a randomised controlled trial of a novel educational booklet in primary care. Spine $\underline{24}, 1-8$

Chenot JF (2010): Qualitätsindikatoren für die Versorgung von Patienten mit Rückenschmerzen. Schmerz $\underline{3}, 24:$ 213-220

Chenot JF, Becker A, Leonhardt C, Keller S, Donner-Banzhoff N, Baum E, Pfingsten M, Hildebrandt J, Basler HD, Kochen MM (2007): Use of complementary alternative medicine for low back pain consulting in general practice: a cohort study. BMC Complement Alternat Med $\underline{7}$, 42

Chenot JF, Kochen MM, Schmidt CO: Das Einhalten von Leitlinien und die Qualität der ambulanten Versorgung von Rückenschmerzpatienten; in Jan Böcken, Bernard Braun und Juliane Landmann (Hrsg.): Gesundheitsmonitor 2009. Gesundheitsversorgung und Gestaltungsoptionen aus der Perspektive der Bevölkerung. Verlag Bertelsmann Stiftung. Gütersloh 2009 a, 135-155

Chenot JF, Pieper A, Kochen MM, Himmel W (2009 b): Kommunikation und Befundaustausch zwischen Hausärzten und Orthopäden bei Rückenschmerzen. Schmerz 2, 173-179

Darlow B, Fullen BM, Dean S, Hurley DA, Baxter GD, Dowell A (2012): The association between health care professional attitudes and beliefs and the attitudes and beliefs, clinical management, and outcomes of patients with low back pain: A systematic review. Eur J Pain $\underline{16}$, 3-17

DEGAM-Leitlinie Nr. 3: Kreuzschmerzen - Gekürzte Internetfassung, DEGAM und omicron publishing, Düsseldorf, Stand: 2003, http://www.degam.de/leitlinien/LL_Kreuz_Internet.pdf

Abrufdatum: 28.10.2012 
Deutscher Schmerzfragebogen, Deutsche Gesellschaft zum Studium des Schmerzes, http://www.dgss.org/index.php?id=704

Abrufdatum: 20.11.2011

Deyo RA, Diehl AK (1986): Patient satisfaction with medical care for low - back pain. Spine $\underline{11}$, 28-30

Deyo RA, Diehl AK, Rosenthal M (1987): Reducing roentgenography use. Can patient expectations be altered? Arch Intern Med 147, 141-145

Dillmann U, Nilges P, Saile H, Gerbershagen HU (1994): Behinderungseinschätzung bei chronischen Schmerzpatienten. (Pain Disability Index) Schmerz $\underline{8}$, 100-110.

Dionne CE, Von Korff M, Koepsell TD, Deyo RA, Barlow WE, Checkoway H (2001): Formal education and back pain: a review. J Epidemiol Community Health $\underline{55}$, 455-468

Fahland RA, Schmidt CO, Raspe H, Feng YS, Kohlmann T: Epidemiologie und sozioökonomische Bedeutung. In: Hildebrandt J, Pfingsten M (Hrsg.) Rückenschmerz und Lendenwirbelsäule. Elsevier: Urban \& Fischer, München 2011, 6-22

Feuerstein M, Hartzell M, Rogers HL, Marcus SC (2006): Evidence based practice for acute low back pain in primary care: patient outcomes and cost of care. Pain; 124: 140-149

Franks P, Boisseau V (1980): Educational status and health. J Fam Pract $\underline{10}$, 1029-1034

Gerbershagen HU, Korb J, Nagel B, Nilges P: Das Mainzer Stadiensystem zur Schmerzchronifizierung - Mainzer Pain Staging System, o.Verl., o.O., o.J.

Grol R (2001): Successes and Failures in the implementation of evidence-based guidelines for clinical practice (Abstract). Med care $\underline{39}$, 8 Suppl 2: ॥46-54

Grol R, Grimshaw J (2003): From best evidence to best practice: effective implementation of change in patients' care. The Lancet $\underline{362}, 1225-1230$ 
'Handlungsleitlinie Kreuzschmerzen' aus: Empfehlungen zur Therapie von Kreuzschmerzen (3. Auflage) Arzneimittelkommission der deutschen Ärzteschaft, Arzneiverordnung in der Praxis, Band 34, Sonderheft 2, April 2007

http://www.akdae.de/Arzneimitteltherapie/TE/A-Z/PDF_Kurzversion/Kreuzschmerz_k.pdf

Haldeman, S, Dagenais S (2008): A supermarket approach to the evidence-informed management of chronic low back pain. Spine J $\underline{8}, 1-7$

Hauswaldt J, Hummers-Pradier E, Junius-Walker U (2012: Health service use among patients with chronic or multiple illnesses, and frequent attenders. Dtsch Ärztebl Int, 109(47):814-820

Hautzinger M, Bailer M: Allgemeine Depressionsskala. Manual, Beltz Test GmbH, Göttingen, 1993

Heuch I, Hagen K, Heuch I, Nygaard C, Zwart JA (2010): The Impact of Body Mass Index on the Prevalence of Low Back Pain: The HUNT Study. Spine $\underline{35}$, 7: 764-768

Heuch I, Heuch I, Hagen K, Zwart JA (2012): Body Mass Index as a Risk Factor for Developing Chronic Low Back Pain: A Follow-up in the Nord-Trøndelag Health Study. Spine (Phila Pa 1976) (Abstract ohne nähere Angaben)

Hildebrandt J, Pfingsten M (Hrsg.) Rückenschmerz und Lendenwirbelsäule. Elsevier: Urban \& Fischer, München 2011

Hildebrandt J, Pfingsten M (2000): Chronischer Rückenschmerz als interdisziplinäre Aufgabe. Notfall 26, 553-557

Hildebrandt J, Mense S (2001): Rückenschmerzen - ein ungelöstes Problem. Schmerz 15, 411412

http://afag-carre.de/index.php?page=medikamentoese-schmerztherapie

(Abrufdatum 13.09.13)

http://www.arbeitssicherheit.de/de/html/lexikon/224/

(Abrufdatum 13.09.13) 
http://www.erste-hilfe-infos.de/index.php?id=1505

http://www.kreuzschmerzen-rueckenschmerzen.de/
(Abrufdatum: 29.12.2011)

(Abrufdatum: 29.12.2011)

http://www.gesetze-im-internet.de/versmedv/BJNR241200008.html (Abrufdatum 06.05.2013)

http://www.versorgungsleitlinien.de/themen/kreuzschmerz (Abrufdatum 13.09.2013)

Kendrick D, Fielding K, Bentley E, Kerslake R, Miller P, Pringle M (2001): Radiography of the lumbar spine in primary care patients with low back pain: randomised control trial. BMJ $\underline{322}$, 400-405

Kohlmann T, Schmidt C: Epidemiologie des Rückenschmerzes. In: Hildebrandt et al. (Hrsg.) Lendenwirbelsäule Ursachen, Diagnostik und Therapie von Rückenschmerzen. 1. Aufl., Urban \& Fischer, Berlin 2005, 1-11 (=3-14)

Krauth C, Grobe T, Hoopmann M, Schwartz FW, Walter U (2004): Krankheitskosten und Einsparpotentiale. In: Hildebrandt J, Müller G, Pfingsten M (Hrsg.) Die Lendenwirbelsäule. Urban \& Fischer, München, 14-26

Mesrian A, Neubauer E, Pirron P, Schiltenwolf M (2005): Multimodale Schmerztherapie bei chronischen und chronifizierenden Rückenschmerzen. Manuelle Med $\underline{43}$, 85-92

Molzen $\mathrm{HU}$ : Entwicklung und Gebrauchstauglichkeitsprüfung eines elektronischen Dokumentationssystems für die Schmerztherapie. Med. Diss. Ulm, 2007

Müller $M$ und Mitarbeiter: Chirurgie für Studium und Praxis. Medizinische Verlags- und Informationsdienste, Breisach 2010/ 2011

Müller G, Pfingsten M: Vom Symptom zur Krankheit. In: Hildebrandt J, Müller G, Pfingsten M (Hrsg.) Die Lendenwirbelsäule. Urban \& Fischer (Elsevier), München 2004, 55-66 
Nagel B, Pfingsten M, Lindena G, Nilges P: Handbuch: Deutscher Schmerzfragebogen Version 2012.1: http://www.dgss.org/fileadmin/pdf/12_DSF_Manual_2012.2.pdf, Abrufdatum 01.01.2012

Nationale Versorgungsleitlinie Kreuzschmerz - Kurzfassung. Version 1.2. August 2011 Bundesärztekammer (BÄK), Kassenärztliche Bundesvereinigung (KBV), Arbeitsgemeinschaft der Wissenschaftlichen Medizinischen Fachgesellschaften (AWMF).

http://www.versorgungsleitlinien.de/themen/kreuzschmerz, Abrufdatum: 18.03.2012

Niebling W (2004): Rückenschmerz aus Sicht des Hausarztes. In: Hildebrandt J, Müller G, Pfingsten M (Hrsg) Lendenwirbelsäule. München: Urban \& Fischer, S. 540-546

Owen JP, Rutt G, Keir MJ, Spencer H, Richardson D, Richardson A, Barclay C (1990): Survey of general practitioners' opinions on the role of radiology in patients with low back pain. $\mathrm{Br} \mathrm{J}$ Gen Pract $\underline{40}$, 98-101

Pfingsten M, Hildebrandt J, Wille T (2000): Chronifizierungsausmaß von Schmerzerkrankungen. Schmerz $\underline{14}, 10-17$

Pfingsten M, Hildebrandt J (2010): Rückenschmerz. In: Kröner-Herwig B et al. (Hrsg.): Schmerzpsychotherapie (7.Auflage), Springer, Berlin, 431-452

Pfingsten M, Müller G, Chenot JF (2011): Vom Symptom zur Krankheit. In: Hildebrandt J, Pfingsten M (Hrsg.) Rückenschmerz und Lendenwirbelsäule. Elsevier: Urban \& Fischer, München, 103-110

Reisbord LS, Greenland S (1985): Factors associated with self-reported back-pain prevalence: a population-based study. J Chron Dis $\underline{38}, 691-702$

Schers H, Wensing M, Huijsmans Z, van Tulder M, Grol R (2001): Implementation Barriers for General Practice Guidelines on Low Back Pain: A qualitative study. Spine 1, 26: Issue 15: E348-E353 
Schmidt CO, Raspe H, Pfingsten M, Hasenbring M, Basler HD, Eich W, Kohlmann T (2007): Back Pain in the German Adult Population. Spine $\underline{32}$, 2005-2011

Schmidt CO, Chenot JF, Pfingsten M, Fahland RA, Lindena G, Marnitz U, Pfeifer K, Kohlmann T (2010): Assessing a risk tailored intervention to prevent disabling low back pain-protocol of a cluster randomized controlled trial. BMC Musculoskelet Disord 11, 5: 1-7

Schulte E, Hermann K, Berghöfer A, Hagmeister H, Schuh-Hofer S, Schenk M, Kopf A, Vilain M, Martus P, Willich SN et al. (2010): Referral practices in patients suffering from non-malignant chronic pain. Eur J Pain 14, 3: 308.e1-308.e10

Scott NA, Moga C, Harstall C (2010): Managing low back pain in the primary care setting: The know-do gap. Pain Res Manag 15, 6: 392-400

Statistisches Bundesamt, Gesundheitsberichterstattung des Bundes, Heft 7, Chronische Schmerzen, http://edoc.rki.de/documents/rki_fv/reUzuR53Jx9JI/PDF/29qmd3FjnRtQ_66.pdf Abrufdatum 28.10.2012

Von Korff M, Deyo RA, Cherkin D, Barlow W (1993): Back pain in primary care: outcomes at one year. Spine 18, 855-862; auch in: DEGAM-Leitlinie Nr. 3: Kreuzschmerzen 2003

Wall BF, Rae S, Darby SC, Kendall GM (1981): A reappraisal of the genetic consequences of diagnostic radiology in Great Britain. Br J Radiol $\underline{54}, 719-730$

Wegweiser „Berufsunfähigkeitsversicherungen“, http://www.wegweiser-berufsunfaehigkeitsversicherung.de/, Abrufdatum 28.10.2012

WHO: International Classification of Diseases, http://apps.who.int/classifications/icd10/browse/2010/en\#/M50-M54, Abrufdatum: 10.11.2012

Wikipedia „Grad der Behinderung“, http://de.wikipedia.org/wiki/Grad_der_Behinderung Abrufdatum 22.07.2012 


\section{Abbildungsverzeichnis}

Abb. 1 Lokalisation:

Nackenschmerzen - Rückenschmerzen - Kreuzschmerzen

Abb. 2 Lasègue-Test

Abb. 3 Diagnostik von "red flags" und spezifischen Ursachen

(0-2 Wochen nach Erstkonsultation)

Abb. 4 Weitere Diagnostik und Therapie bei akutem nicht-spezifischem

Kreuzschmerz (2-5 Wochen nach Erstkonsultation)

Abb. 5 Versorgung von subakutem Kreuzschmerz

30

(6-12 Wochen nach Erstkonsultation)

Abb. 6 BMI-Profil der Patienten

49

Abb. 7 Klassifizierte Werte der Allgemeinen Depressions-Skala (ADS)

52

Abb. 8 Verordnete Maßnahmen im Erstkontakt

57

Abb. 9 Verschriebene Medikamente

58

Abb. 10 Erinnerte Information zur Verordnung der Medikamente

60

Abb. 11 Häufigkeit von Rehabilitationsaufenthalten

61

Abb. 12 Von den Befragten angegebene therapeutische Maßnahmen

63

Abb. 13 Eigens angewendete Maßnahmen

64

Abb. 14 Weiterbehandelnde Arztgruppen 
Abb. 15 In orthopädischen Praxen durchgeführte/ verordnete Therapien

Abb. 16 Neurochirurgische Therapiemaßnahmen

68

Abb. 17 Zufriedenheit mit orthopädischer Behandlung (Noten von 1-5)

71

Abb. 18 Zufriedenheit mit neurochirurgischer Behandlung (Noten1-5)

72

Abb. 19 WHO-Stufenschema der medikamentösen Schmerztherapie

93 


\section{Tabellenverzeichnis}

Tab.1 Warnhinweise auf eine spezifische vertebragene Ursache („red flags“)

Tab. 2 Anzahl der radiologisch ermittelten Diagnosen pro Patient

Tab. 3 Depressive Symptome, Beeinträchtigungserleben und subjektive

Schmerzintensität bei Patienten mit der Empfehlung, eine

Psychotherapie aufzunehmen im Vergleich zu Patienten ohne eine solche Empfehlung

Tab. 4 Vergleich der Zufriedenheits-Werte mit allen beteiligten Facharzt-Gruppen 72 
Zentrum Anaesthesiologie, Rettungs- und Intensivmedizin

Schmerz-Tagesklinik und -Ambulanz

Kommissar. Leitung

Prof. Dipl. Psych. Dr. Michael Pfingsten (Itd. Psychologe)

Dr. med. Joachim Strube (Oberarzt)

Robert-Koch-Str.40,37075Göttingen

Tel:0551/398816

Fax: 0551/39 4164

E-Mail: pain@med.uni-goettingen.de

\section{Patienten-Information}

\section{Studie: „Patientenwege von Patienten mit nicht-spezifischen Rückenschmerzen“}

\section{Die Teilnahme an der nachfolgend beschriebenen Studie ist freiwillig!}

\section{Worum geht es bei unserer Untersuchung?}

Rückenschmerzen sind ein häufiger Anlass für einen Arztbesuch. Bei den meisten Betroffenen handelt es sich um unkomplizierte Verläufe und die Beschwerden klingen rasch wieder ab. Es gibt aber auch Patienten, bei denen sich ein chronischer Schmerz entwickelt und der Verlauf langwieriger ist. Diese Patienten werden dann oftmals auch in Schmerztherapeutischen Einrichtungen behandelt.

Über die Behandlungswege von Patienten mit chronischen Rückenschmerzen ist in Deutschland noch nicht sehr viel bekannt: So ist unklar, zu welchen Ärzten sie gehen, welche diagnostischen und therapeutischen Maßnahmen sie dort erhalten und welchen Erfolg diese aus ihrer Sicht haben. Auch die Frage, wann welcher Patient zum Facharzt überwiesen wird und aus welchen Gründen dies geschieht, ist noch nicht gut untersucht. Eine Verbesserung der Abläufe könnte den Patienten helfen, dass Abläufe beschleunigt werden, den Patienten schneller geholfen werden kann und letztlich auch die finanziellen Mittel sinnvoller eingesetzt werden können. Um diese Abläufe optimieren zu können, muss man sie aber erst einmal kennen lernen und analysieren.

Diese wissenschaftliche Untersuchung hat das Ziel, die Vorbehandlung von Patienten mit chronischen Rückenschmerzen zu erfassen, zu analysieren und Vorschläge für eine Veränderung zu machen.

\section{Bei dieser Untersuchung bitten wir Sie sehr herzlich um Ihre Mitarbeit!}

Wir wollen Ihre Behandlungsgeschichte anhand der vorliegenden Arztbriefe und Befundberichte analysieren. Diese wollen wir im Zeitverlauf tabellarisch auflisten und die durchgeführten Maßnahmen erfassen. Nachdem wir den Verlauf anhand der Briefe/ Berichte zusammengestellt haben, wollen wir das Ergebnis mit Ihnen besprechen und Ihnen u.a. Fragen nach Zeitabläufen und Erfolgen dieser Maßnahmen stellen. Als Mehraufwand zusätzlich zu Ihrer Behandlung fällt nur dieses einmalige Gespräch von ca. 45min Dauer an, das die Doktorandin Frau Helbing an einem Ihrer ersten 3 Behandlungstage bei uns mit Ihnen führen wird. Frau Helbing wird sich über das Gespräch Notizen machen und die Daten mit den Informationen aus den Arztbriefen und Berichten abgleichen. Mehr Aufwand ist für Sie nicht erforderlich.

Selbstverständlich ist die Teilnahme an dieser Untersuchung freiwillig. Ihr Arzt wird Sie unabhängig von der Befragung und deren Ergebnissen beraten und behandeln. Sie können Ihre Einwilligung zur Teilnahme jederzeit widerrufen, ohne dass Ihnen dadurch Nachteile entstehen.

\section{Datenschutz!}

Alle Angaben unterliegen der Schweigepflicht. Ihre persönlichen Daten (z.B. Name und Adresse) tauchen nur während der Datensammlung in entsprechenden Tabellen auf. Bevor die Daten zur Auswertung auf einem Computer gespeichert werden, sind sie pseudonomisiert, d.h. die Namen werden nicht auf den Computer übertragen. Die Daten werden nicht an Dritte weitergegeben, sondern nur von Frau Helbing bzw. Prof. Pfingsten zur Auswertung genutzt. Tabellen und Daten bleiben in der Schmerztagesklinik und Ambulanz. Wenn Sie Ihre Studienteilnahme im Verlauf widerrufen, werden die erhobenen Aufzeichnungen aus dem Gespräch vernichtet. Die anonymisierten/ pseudonomisierten Daten können dann nicht mehr gelöscht werden, eine Zuordnung zu Ihrem Namen ist aber ausgeschlossen. 


\section{Einverständniserklärung}

Studie: „Patientenwege von Patienten mit nicht-spezifischen Rückenschmerzen“

Ich bin über das o.g. Forschungsvorhaben der Schmerztagesklinik und -Ambulanz in der Universitätsmedizin Göttingen ausreichend informiert worden und erkläre mich bereit, daran teilzunehmen.

Die Teilnahme an dieser Studie ist freiwillig und kann jederzeit von mir beendet werden. Sofern ich nicht an dieser Studie teilnehmen oder später aus ihr ausscheiden möchte, erwachsen mir daraus keine Nachteile für meine Behandlung.

Ich bin damit einverstanden, dass meine Angaben in den Fragebögen sowie die Ergebnisse der ärztlichen Untersuchung ausschließlich für wissenschaftliche Zwecke gespeichert und ausgewertet werden. Nach Beendigung des Forschungsvorhabens werden alle Daten gelöscht, die einen Bezug zu meiner Person erlauben. Eine Weitergabe an Dritte ist ausgeschlossen.

Die von mir unterzeichnete Einverständniserklärung wird getrennt von meinen Angaben aufbewahrt, so dass eine Zuordnung von Namen und persönlichen Angaben nicht möglich ist.

Das Informationsblatt und den Text dieser Erklärung habe ich erhalten.

Name, Vorname:

Geburtsdatum:

Adresse:

Straße, Hausnummer

PLZ, Wohnort

Ort, Datum

Unterschrift 


\section{Standardisierte Erfassung:}

Nachname:

Vorname:

Datum der Erstvorstellung in der Schmerzklinik:

Aus Deutschem Schmerz-Fragebogen:

\begin{tabular}{|l|l|}
\hline Körpergröße: & \\
\hline Körpergewicht & \\
\hline BMI & \\
\hline Personenstand & \\
\hline Schulabschluss & \\
\hline Ausbildungsstand & \\
\hline Akt. berufl. Situation & \\
\hline Laufendes Rentenverfahren, MdE & \\
\hline Psychometrische Fragebogen (ADS, & \\
PDI) & \\
\hline Schmerzintensität (Ø, max.) & \\
\hline Vor-Operationen & \\
\hline
\end{tabular}

Aus ärztlicher Anamnese:

Chronifizierungsstatus (MPSS):

Diagnose der Schmerzklinik:

Aus psychologischer Exploration:

Hinweise auf Wirksamkeit psychosozialer Faktoren: $\mathrm{O}$ ja, stark $\mathrm{O}$ ja, mäßig $\mathrm{O}$ nein 


\section{Patientenbefragung (Leitfaden):}

0. Erstmanifestation der Rückenschmerzen:

1. Arztkontakt wg. aktueller Episode:
O wenige Tage
O 1 Woche
O 1 Monat
O 1-2 Monate
O 3 Monate
O 4-5 Monate
O 6 Monate

nach dem 1. Auftreten des Schmerzes

2. Fachgebiet des zuerst aufgesuchten Arztes:

3. Diagnostische Erstmaßnahmen:

Sind Sie körperlich untersucht worden?

O ja O nein

Mussten Sie Ihre Kleidung ablegen?

O ja O nein

Wurde Ihre Wirbelsäule abgetastet?

O ja O nein

Wurden Ihre Reflexe getestet?

O ja O nein

Welche Untersuchungen sind Ihnen sonst noch bekannt?

4. Yellow flags (psycho-soziale Risikofaktoren). Sind Fragen gestellt worden ....

O zur beruflichen Situation (Arbeitsplatzmerkmale)

O zur familiären/ partnerschaftlichen Lebenssituation

O zum Umgang mit Schmerz (ggf. Gedanken/ Überzeugungen)

5. Wurden diese Merkmale im späteren Behandlungsverlauf erfasst?

O ja

O nein wenn ja, durch wen: 
6. Bildgebende Diagnostik

a) Ist beim ersten Arztkontakt ein bildgebendes Verfahren durchgeführt bzw. initiiert worden?

$$
\mathrm{O} \text { ja } \mathrm{O} \text { nein }
$$

Wenn ja, folgendes Verfahren:
a. Röntgen,
$\mathrm{O}$
b. Computertomographie
$\mathrm{O}$
c. Magnetresonanztomographie/ NMR O
d. Anderes
$\mathrm{O}$

b) Von wem ging die Initiative zur Durchführung aus?
O von mir selbst
O vom Hausarzt
O vom Orthopäden
$\mathrm{O}$
O es wurde kein bildgebendes Verfahren durchgeführt

c) Wie häufig sind diese Verfahren wegen Ihrer Rückenschmerzen in den letzten 3 Jahren durchgeführt worden?
a. Röntgen, mal
b. Computertomographie mal
c. Magnetresonanztomographie/ NMR mal
d. anderes mal

d) Falls bildgebende Verfahren angewandt wurden, wurde der radiologische Befund mit Ihnen besprochen?

O nein, es wurden nur die CD/ Bilder ausgehändigt.

O es wurde gesagt, dass eine Schädigung am Rücken vorläge

O es wurde gesagt, dass alles weitgehend normal sei 
O ich habe darauf bestanden, andernfalls wäre der Befund nicht besprochen worden

7. Wurde eine Diagnose mitgeteilt?

$\mathrm{O}$ ja

O nein

Welche?: durch wen?

Welche?: durch wen?

Welche?: durch wen?

8. Therapie

a) Therapie des zuerst behandelnden Arztes:

b) Ist Ihnen die Prognose/ der Verlauf erläutert worden?

$\mathrm{O} \mathrm{ja}$

O nein Wenn ja, durch wen?

c) Wurde Ihnen geraten, körperlich möglichst aktiv zu bleiben?

O ja O nein Wenn ja, durch wen?

d) Wurde Physiotherapie verschrieben? O ja $\quad \mathrm{O}$ nein Anzahl der Termine:

e) An welche Medikamente können Sie sich erinnern? 
f) Haben Sie frei verkäufliche Schmerzmittel eingenommen

O nein

Ja, welche :

Dosierung

regelmäßig

bei Bedarf

$\mathrm{O}$

$\mathrm{O}$

$\mathrm{O}$

$\mathrm{O}$

g) Nehmen Sie immer noch zusätzlich frei verkäufliche Schmerzmittel ein O nein Ja, welche :

Dosierung regelmäßig

bei Bedarf

$\mathrm{O}$

$\mathrm{O}$

$0 \quad 0$

h) Wenn Ihnen ein Schmerzmittel verordnet wurde:

O nein Ist Ihnen die Dosierung erläutert worden?

O ja O nein

Ist Ihnen die Einnahmedauer erklärt worden

O ja O nein

Sind Nebenwirkungen besprochen worden

O ja O nein

i) Haben Sie Nebenwirkungen von Schmerzmedikamenten erlebt?

O nein Ja, welche:

j) Wurden im Behandlungsverlauf neben der Verordnung von Schmerzmedikamente noch andere Behandlungsmaßnahmen durchgeführt?

durch wen?:

durch wen?:

durch wen?: 
k) Wie häufig sind diese Verfahren die letzten 3 Jahre durchgeführt worden?

verordnender Arzt:

a. Verordnung von Einlagen

mal,

b. Magnetmatte mal,

c. Reizstrom mal,

d. Injektionen in den Rücken ohne Bildgebung mal,

e. Injektionen in Gesäß mal,

f. Injektionen in den Rücken mal,

g. Manipulationen (Einrenken) mal,

h. Rückenschule (Kasse) mal,

i. TENS mal,

j. Infusionen von Schmerzmitteln mal,

k. Kur/ Reha-Maßnahme mal,

I. Anderes mal,

I) Haben Sie eigene Maßnahmen durchgeführt und z.B. etwas gekauft? (neue Matratze, Massagen, Yoga, Hypnose, Fango, Wassergymnastik, regelmäßiger Sport z.B. Fitnessstudio oder schwimmen, Heimtrainer, etc.)

O nein

$\mathrm{O} \mathrm{ja}$

m) Haben Sie für Behandlungen selbst gezahlt?

Wie viel Geld haben Sie schätzungsweise selbst für die Behandlung Ihrer Rückenschmerzen ausgegeben? 
- selbstbezahlte Massagen

O ja O nein

- Osteopathie

$\mathrm{O}$ ja

O nein

- Rückenschule (privat)

O ja $\quad O$ nein

9. Wurden Sie krank geschrieben? O ja O nein wenn ja, wie lange? Wochen

(O nicht Berufstätig) Monate Jahr/ e

\section{Weiterer Behandlungsverlauf}

10. Bei wem waren Sie sonst noch wegen Ihrer Rückenschmerzen in Behandlung?

O Orthopäde: Anzahl Termine/ Zeitraum: Zufriedenheit (1-5):

Therapie:

O Neurologe: Anzahl Termine / Zeitraum Zufriedenheit (1-5):

Therapie:

O Neurochirurg: Anzahl Termine / Zeitraum Zufriedenheit (1-5): Therapie:

O Heilpraktiker: Anzahl Termine / Zeitraum Zufriedenheit (1-5): Therapie:

$\mathrm{O}$ :Anzahl Termine/ Zeitraum Zufriedenheit (1-5):

11. Wurde jemals eine psychotherapeutische Behandlung empfohlen oder durchgeführt?

Örztlich empfohlen und durchgeführt

O ärztlich empfohlen und begonnen, aber nicht kontinuierlich weitergeführt

Örztlich empfohlen, aber nicht begonnen

O nicht ärztlich empfohlen 
12. Ist eine Kur / Rehabilitation beantragt / durchgeführt worden? $O$ ja $O$ nein Klinik:

Aufenthaltsdauer:

Erfolg (1-5):

Klinik:

Aufenthaltsdauer: Erfolg (1-5): Klinik:

Aufenthaltsdauer: Erfolg (1-5):

\section{Allgemeine Fragen}

13. Haben Sie während Ihres Krankheitsverlaufs jemals schriftliche Informationen bekommen zu:

Rückenschmerzen $\quad$ ja $\quad$ nein wenn ja, von wem?

lokalen Angeboten von Bewegungsprogrammen

O ja O nein wenn ja, von wem?

14. Hatten Sie während Ihres Krankheitsverlaufs das Gefühl, dass ein bestimmter Arzt alles koordiniert? O nein $\mathrm{O}$ ja Wenn ja, welches Fachgebiet?

15. Wurden mit diesem Ihre Diagnosen bzw. die diagnostische Ergebnisse mitbehandelnder Fachärzte besprochen?

$O \mathrm{ja}$, in regelmäßigen Abständen $\mathrm{O}$ selten $\quad \mathrm{O}$ einmal $\quad \mathrm{O}$ nie O ich habe keinen Arzt, der alles koordiniert

16. Haben Sie während Ihres Krankheitsverlaufs Ihre Ärzte gewechselt? $\mathrm{O}$ ja O nein Gründe: O wegen Umzug

$\mathrm{O}$ aus Unzufriedenheit

O andere: 
17. Von wem wurden Sie in die Schmerzklinik überwiesen?

Bemerkungen: 
Das Mainzer Stadienmodell der Schmerz-Chronifizierung (MPSS) Auswertungsformular

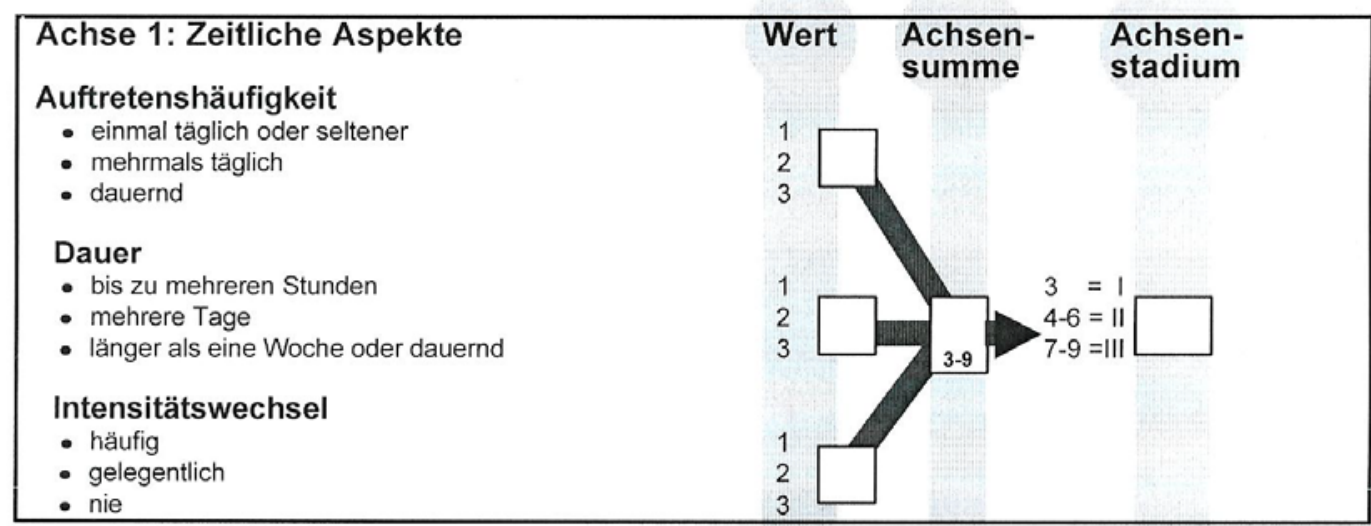

Achse 2: Räumliche Aspekte
Schmerzbild
$\begin{aligned} & \text { - monolokulär } \\ & \text { - bilokulär } \\ & \text { - multilokulär oder Panalgesie }\end{aligned}$

Achse 3: Medikamenteneinnahmeverhalten
Medikamenteneinnahme
- unregelmäßiger Gebrauch von
max.2 peripheren Analgetika
max. 3 periphere Analgetika,
$\quad$ höchstens 2 regelmäßig
$\quad$ regelmäßig mehr als 2 periphere Analgetika
oder zentralwirkende Analgetika
$\begin{aligned} & \text { Anzahl der Entzugsbehandlungen } \\ & \text { - keine } \\ & \text { - eine }\end{aligned}$

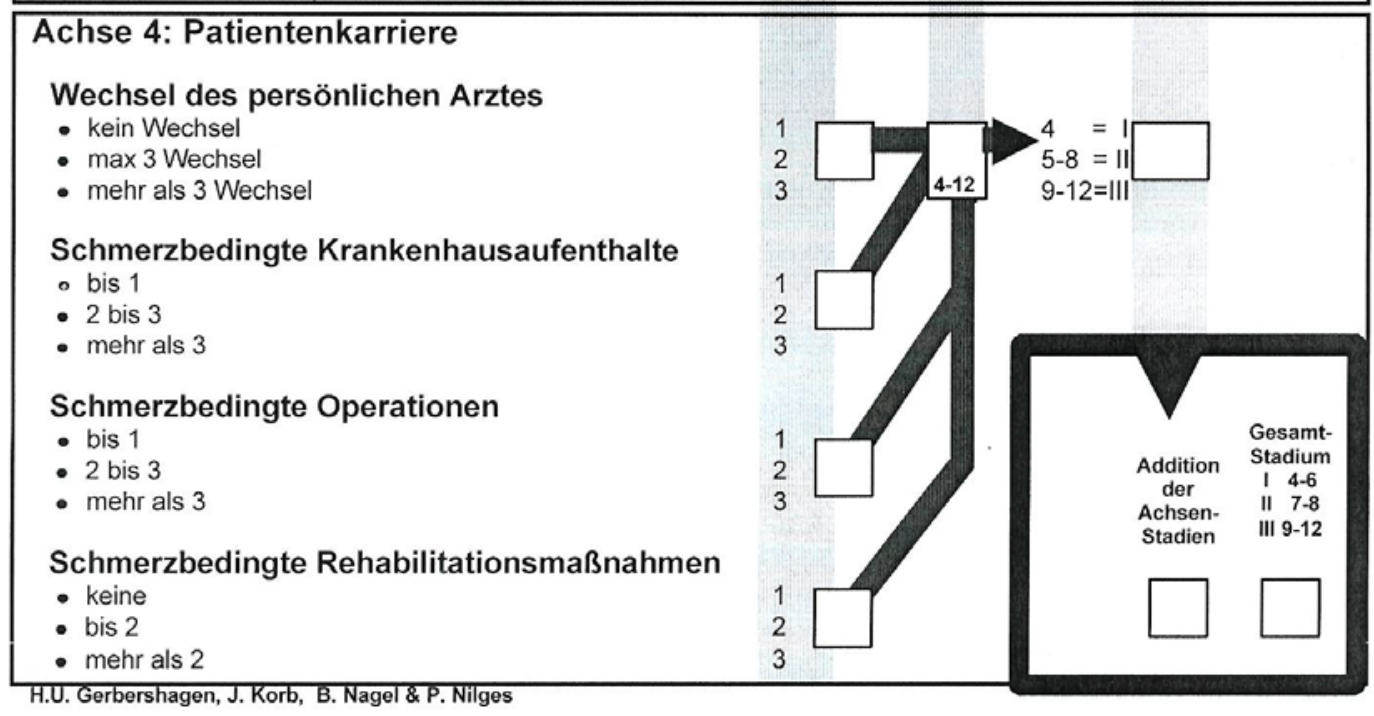




\section{Deutscher Schmerz-Fragebogen}

Stempelfeld Schmerztherapeutische Einrichtung

\section{Empfohlen von}

Deutsche Gesellschaft für Schmerztherapie e.V.

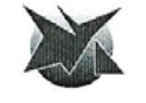

German Pain Association. Société Allemande de la Douleur

und

DGSS

DEUTSCHE GESELLSCHAFT ZUM STUDIUM DES SCHMERZES E.V.

Copyright: www.dgss.org 
Sehr geehrte Patientin, sehr geehrter Patient,

sicher haben Sie selbst schon erlebt wie schwierig es ist, Ihrem Arzt eine genaue Beschreibung Ihrer Schmerzen zu geben. Er ist jedoch darauf angewiesen, zu erfahren, wie Ihre Schmerzen beschaffen sind und wie Sie auf die Schmerzbehandlung ansprechen.

Ihre Angaben in diesem Fragebogen dienen der Vorbereitung des ersten ärztlichen Gespräches und der Basisuntersuchung. In Ihrem eigenen Interesse möchten wir Sie deshalb bitten, alle Fragen in Ruhe und mit Sorgfalt zu beantworten.

Bitte beantworten Sie alle Fragen, auch die, die Ihnen unwichtig erscheinen. Wenn Ihnen eine Frage unklar ist, machen Sie bitte vor der entsprechenden Frage ein Fragezeichen.

Ihre Daten unterliegen der ärztlichen Schweigepflicht. Die Bearbeitung Ihrer Angaben in unserer schmerztherapeutischen Einrichtung entspricht den Bestimmungen des Bundesdatenschutzgesetzes.

Ihre Fragebogendaten werden in einen EDV-Dokumentationssystem gespeichert, das nur berechtigten Mitarbeitern unserer Abteilung zugănglich ist. Diese sind zur Verschwiegenheit verpflichtet.

Zur Verbesserung der Versorgung werden Daten für eine externe Qualitätssicherung herangezogen. Dabei werden die Daten pseudonymisiert, d.h. sie können Ihnen nicht zugeordnet werden.

Wir bitten Sie hiermit um Ihr Einverständnis zur Abspeicherung der Daten. Dieses Einverständnis können Sie jeder Zeit widerrufen.

\title{
Einverständniserklärung
}

Ich bin mit der Abspeicherung und der pseudonymisierten Verwendung der im Fragebogen erhobenen Daten einverstanden.

Die folgenden vier Zeilen bitte nicht ausfüllen!

\author{
Patienten-Nr: \\ Ausgabe-Datum: \\ Eingangs-Datum: \\ Erster Behandlungstermin:
}


Patient: Datum beim Ausfüllen:

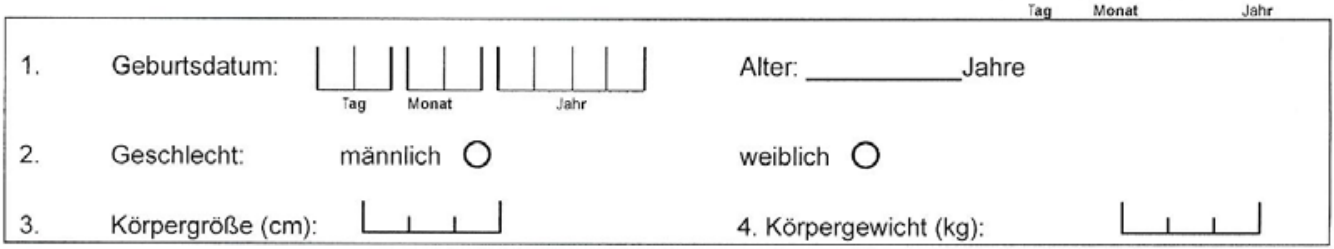

5. Bitte zeichnen Sie im Körperschema ein, an welchen Körperstellen Ihre Schmerzen auftreten

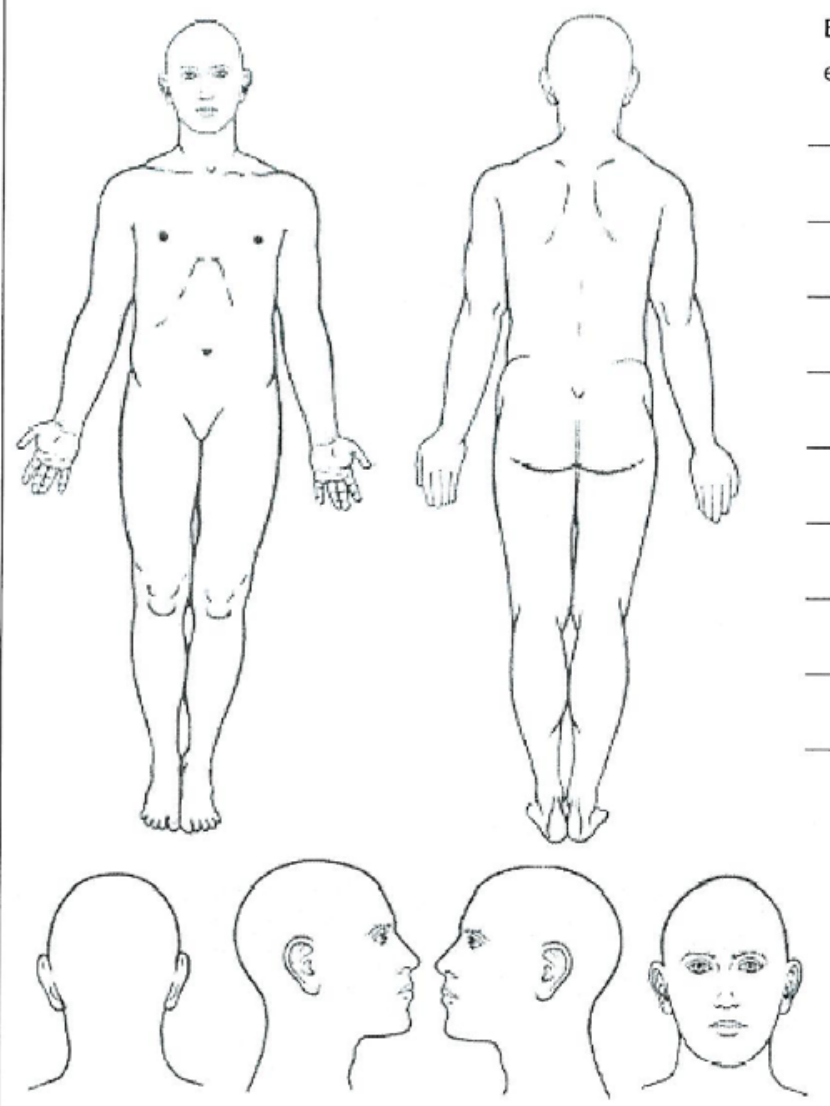
Bitte beschreiben Sie Ihre Schmerzen mit

eigenen Worten:

6. Wegen welcher Schmerzen kommen Sie hauptsächlich zur Behandlung?

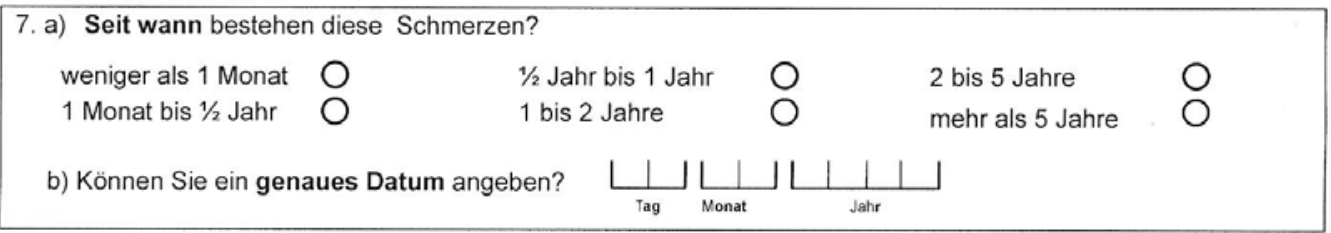


8. a) Welche der Aussagen trifft auf Ihre Schmerzen in den letzen 4 Wochen am besten zu? (Bitte nur eine Angabe machen!)

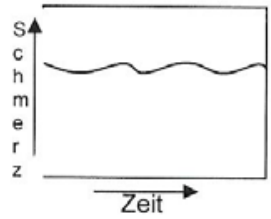

1) Dauerschmerzen mit leichten Schwankungen $\bigcirc$

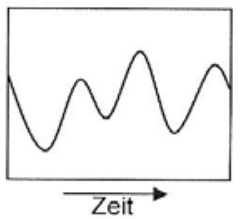

2) Dauerschmerzen mit starken Schwankungen $\mathrm{O}^{\circ}$

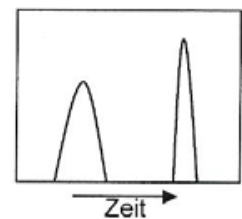

3) Schmerzattacken, dazwischen schmerzfrei $\mathrm{O}^{\circ}$

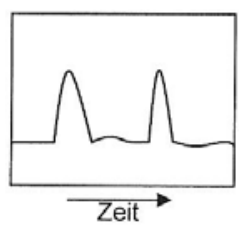

4) Schmerzattacken, auch dazwischen Schmerzen

Wenn Sie an Schmerzattacken leiden (Bilder 3 und 4), beantworten Sie bitte zusätzlich noch folgende Fragen:

b) Wie oft treten diese Attacken durchschnittlich auf?

$\begin{array}{lllll}\text { mehrfach täglich } & \bigcirc & \text { einmal täglich } & \bigcirc & \text { mehrfach wöchentlich } \bigcirc \\ \text { einmal wöchentlich } & \bigcirc & \text { mehrfach monatlich } & \bigcirc & \text { einmal monatlich } \\ \text { seltener: } & \bigcirc & & & \end{array}$

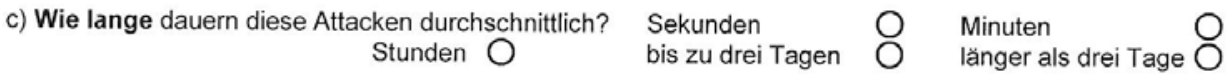
9. Sind Ihre Schmerzen zu bestimmten Tageszeiten besonders stark?
wenn ja: morgens $\mathrm{O}$ mittags $\mathrm{O}$ nachmittags $\mathrm{O}$
abends $\mathrm{O}$ nein $\bigcirc$

10. Mit der folgenden Liste von Eigenschaftsworten können Sie genauer beschreiben, wie Sie Ihre Schmerzen empfinden. Denken Sie bei der Beantwortung an Ihre typischen Schmerzen in der letzten Zeit. Bitte lassen Sie keine der Beschreibungen aus und machen Sie für jedes Wort ein Kreuz, inwieweit die Aussage für Sie zutrifft.

Sie haben bei jeder Aussage 4 Antwortmöglichkeiten:

$3=$ trifft genau $z \mathrm{u} \quad 2=$ trifft weitgehend $\mathrm{zu} \quad 1=$ trifft ein wenig zu $\quad 0=$ trifft nicht $z \mathrm{u}$

Ich empfinde meine Schmerzen als ....

\begin{tabular}{|c|c|c|c|c|c|c|c|c|c|}
\hline & $\begin{array}{c}\text { trifft } \\
\text { genau } \\
\text { zu }\end{array}$ & $\begin{array}{l}\text { trifft weit- } \\
\text { gehend } \\
\mathrm{zu}\end{array}$ & $\begin{array}{l}\text { trifft ein } \\
\text { wenig } \\
\mathrm{zu}\end{array}$ & $\begin{array}{l}\text { trifft } \\
\text { nicht } \\
\mathrm{zu}\end{array}$ & & $\begin{array}{c}\text { trifft } \\
\text { genau } \\
\text { zu }\end{array}$ & $\begin{array}{l}\text { trifft weit- } \\
\text { gehend } \\
\mathrm{zu}\end{array}$ & $\begin{array}{l}\text { trifft ein } \\
\text { wenig } \\
\mathrm{zu}\end{array}$ & $\begin{array}{c}\text { trifft } \\
\text { nicht } \\
\mathrm{zu}\end{array}$ \\
\hline & 3 & 2 & 1 & 0 & & 3 & 2 & 1 & 0 \\
\hline ....dumpf & $\mathrm{O}$ & 0 & $\mathrm{O}$ & $\mathrm{O}$ & ....heiß & $\mathrm{O}$ & $\mathrm{O}$ & $\mathrm{O}$ & $\mathrm{O}$ \\
\hline ....drückend & 0 & 0 & $\mathrm{O}$ & 0 & ....brennend & $\mathrm{O}$ & 0 & 0 & 0 \\
\hline ....pochend & 0 & $\mathrm{O}$ & $\mathrm{O}$ & 0 & ....elend & 0 & $\mathrm{O}$ & 0 & $\mathrm{O}$ \\
\hline ....klopfend & 0 & $\mathrm{O}$ & $\mathrm{O}$ & $\mathrm{O}$ & ....schauderhaft & 0 & 0 & 0 & 0 \\
\hline ....stechend & $\mathrm{O}$ & $\mathrm{O}$ & $\mathrm{O}$ & $\mathrm{O}$ & ....scheußlich & 0 & $\mathrm{O}$ & 0 & $\mathrm{O}$ \\
\hline .... ziehend & $\mathrm{O}$ & $\mathrm{O}$ & $\mathrm{O}$ & 0 & ....furchtbar & $\mathrm{O}$ & 0 & $\mathrm{O}$ & $\mathrm{O}$ \\
\hline
\end{tabular}


11. Geben Sie im Folgenden die Stärke Ihrer Schmerzen an. Kreuzen Sie auf den unten aufgeführten Linien an, wie stark Sie Ihre Schmerzen empfinden (unter Ihrer üblichen Medikation). Die Zahlen können Ihnen bei der Einteilung helfen: Ein Wert von 0 bedeutet, Sie haben keine Schmerzen, ein Wert von 10 bedeutet, Sie leiden unter Schmerzen, wie sie für Sie nicht stärker vorstellbar sind. Die Zahlen dazwischen geben Abstufungen der Schmerzstärke an.

a) Geben Sie bitte zunächst Ihre momentane Schmerzstärke an:

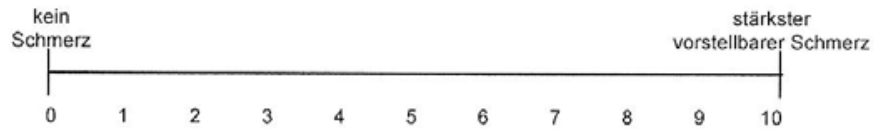

b) Geben Sie jetzt bitte Ihre durchschnittliche Schmerzstärke während der letzten 4 Wochen an:

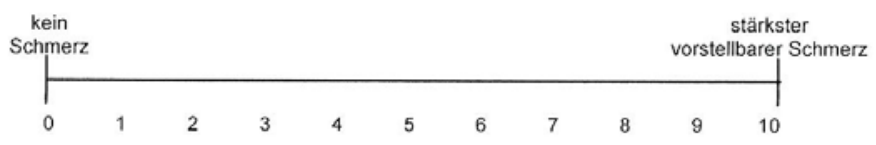

c) Geben Sie jetzt bitte Ihre größte Schmerzstärke während der letzten 4 Wochen an:

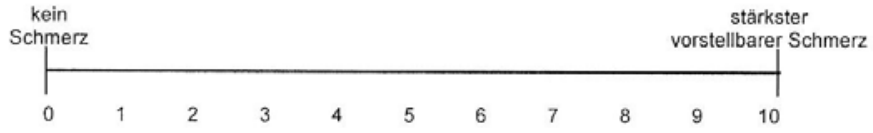

d) Geben Sie jetzt an, welche Schmerzstärke für Sie bei erfolgreicher Behandlung erträglich wäre:

\begin{tabular}{|llllllllllll} 
kein \\
Schmerz
\end{tabular}

12. In den folgenden Fragen geht es um Ihre Schmerzen während der letzten 3 Monate. Für diesen Zeitraum möchten wir Genaueres über die Auswirkungen der Schmerzen erfahren.

a) An wie vielen Tagen konnten Sie in den letzten 3 Monaten aufgrund von Schmerzen nicht Ihren üblichen Aktivitäten nachgehen (z.B. Beruf, Schule, Haushalt)?

$$
\text { an etwa } L \perp 」 \text { Tagen }
$$

b) In welchem Maße haben die Schmerzen in den letzten 3 Monaten Ihren Alltag (Ankleiden, Waschen, Essen, Einkaufen etc.) beeinträchtigt?

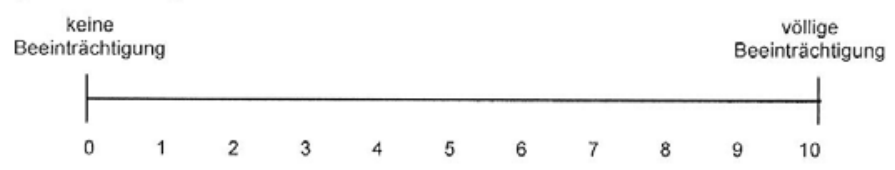

c) In welchem Maße haben die Schmerzen in den letzten 3 Monaten Ihre Freizeitaktivitäten oder Unternehmungen im Familien- oder Freundeskreis beeinträchtigt?

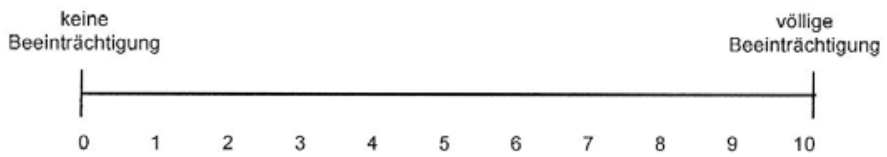

d) In welchem Maße haben die Schmerzen in den letzten 3 Monaten Ihre Arbeitsfähigkeit (einschließlich Hausarbeit) beeinträchtigt?

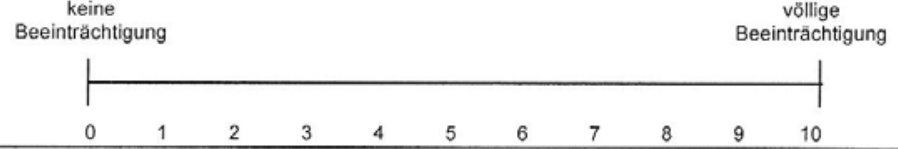


13. a) Auf welche Ursachen führen Sie Ihre Schmerzen zurück? (Mehrfachnennungen sind möglich)

für mich ist keine Ursache erkennbar $\bigcirc$

auf eine bestimmte Krankheit

wenn ja, welche?

auf eine Operation

wenn ja, welche?

Datum der Operation

$\left.\bigsqcup_{\text {Tag }}\right\rfloor \bigsqcup_{\text {Monat }} \perp \perp \perp|\perp|$

auf einen Unfall

wenn ja, welchen?

Datum des Unfalls

auf körperliche Belastung

O

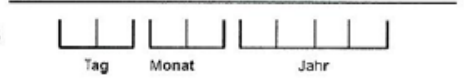

auf seelische Belastung

O

auf eine andere Ursache

wenn ja, welche?

Falls Ihre Schmerzen im Zusammenhang mit einem Unfall, einer berufsbedingten Erkrankung oder Ersatzansprüchen (z.B. nach Operationen) stehen:

b) Sind alle diesbezüglichen rechtlichen oder versicherungsrechtlichen Fragen abgeschlossen

(z.B. Schmerzensgeld)?

ja

nein

$\mathrm{O}$

14. Was machen Sie selbst, um Ihre Schmerzen günstig zu beeinflussen?

Bitte machen Sie genaue Angaben, z.B. spazieren gehen, schlafen, Ablenkung, ...

Ich kann meine Schmerzen nicht beeinflussen $\quad \bigcirc$

15. Was löst Ihrer Erfahrung nach die Schmerzen aus oder verschlimmert sie?

Ich weiss es nicht

16. Bitte schätzen Sie Ihr derzeitiges allgemeines Wohlbefinden ein. Geben Sie bitte an, wie Sie sich in den letzten 14 Tagen meistens gefühlt haben. Kreuzen Sie dazu auf der 6-stufigen Skala jeweils die Zahl an, die am ehesten auf Sie zutrifft: 0 = trifft gar nicht zu, $5=$ trifft vollkommen zu. Bearbeiten Sie bitte alle Aussagen

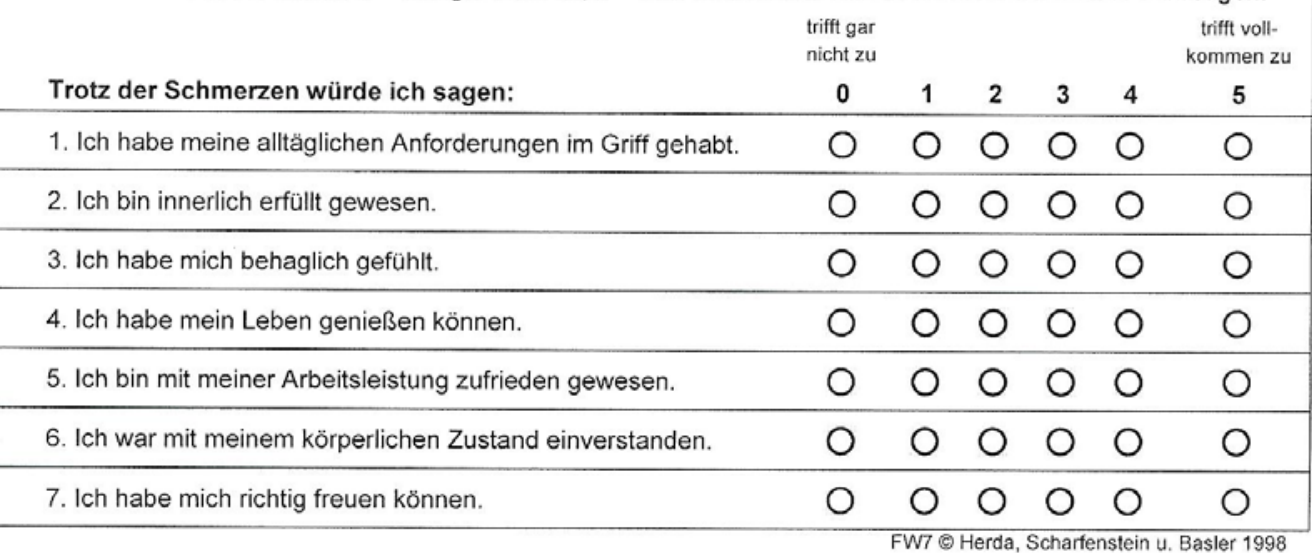


17. Zur vollständigen Beurteilung ihrer Erkrankung bitten wir Sie nun um einige persönliche Angaben. Man weiß heute, dass körperliche Krankheit und seelisches Befinden oft eng zusammenhängen. Deshalb beziehen sich die Fragen ausdrücklich auf Ihre allgemeine und seelische Verfassung

Wir bitten Sie, jede Frage zu beantworten, und zwar so, wie es für Sie persönlich in den letzten 14 Tagen

(inklusive heute) am ehesten zutraf bzw. zutrifft. Machen Sie bitte ein Kreuz für jede Feststellung und lassen Sie bitte keine aus. Überlegen Sie nicht lange, sondern wählen Sie die Antwort aus, die Ihnen auf Anhieb am zutreffendsten erscheint.

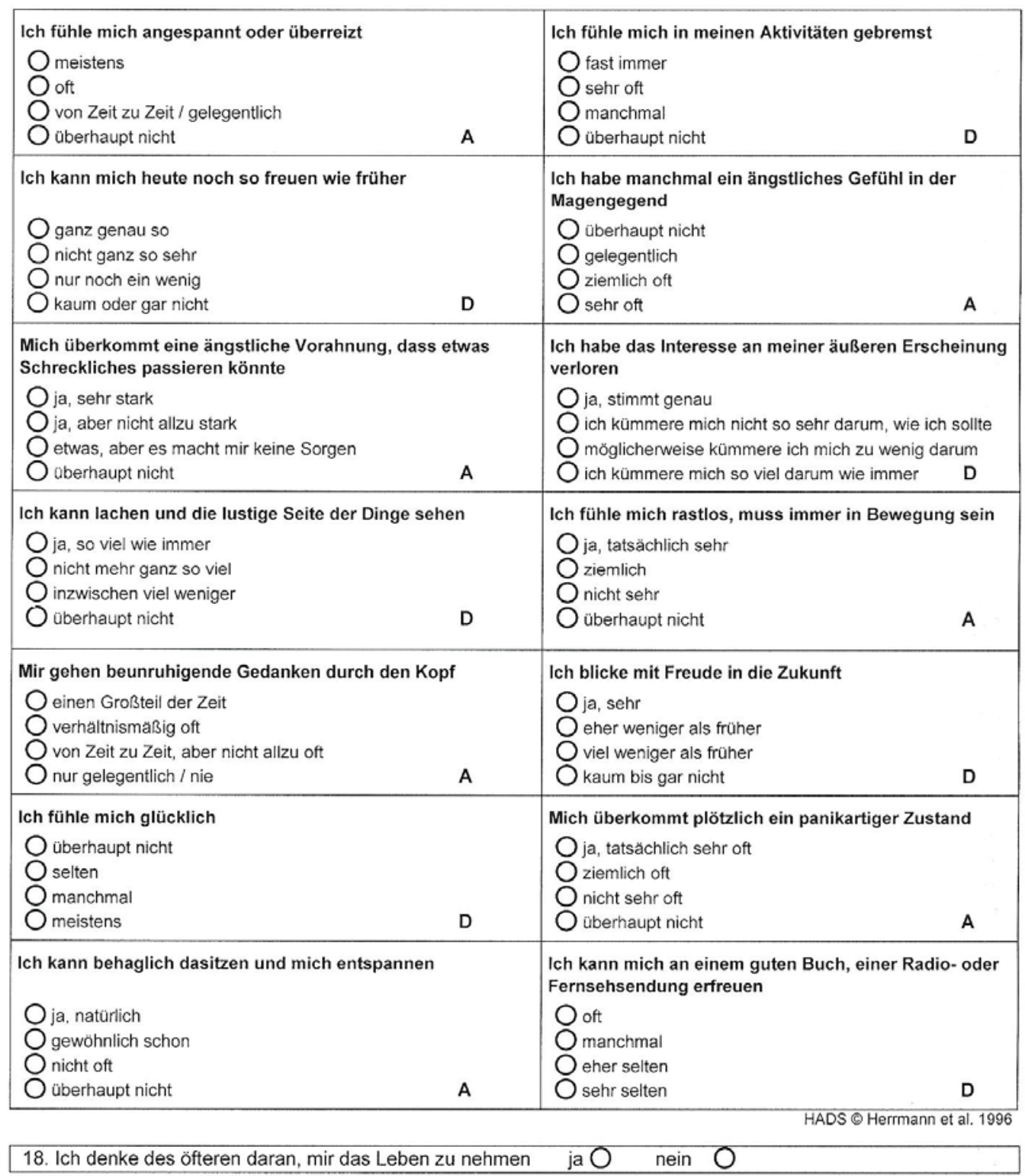




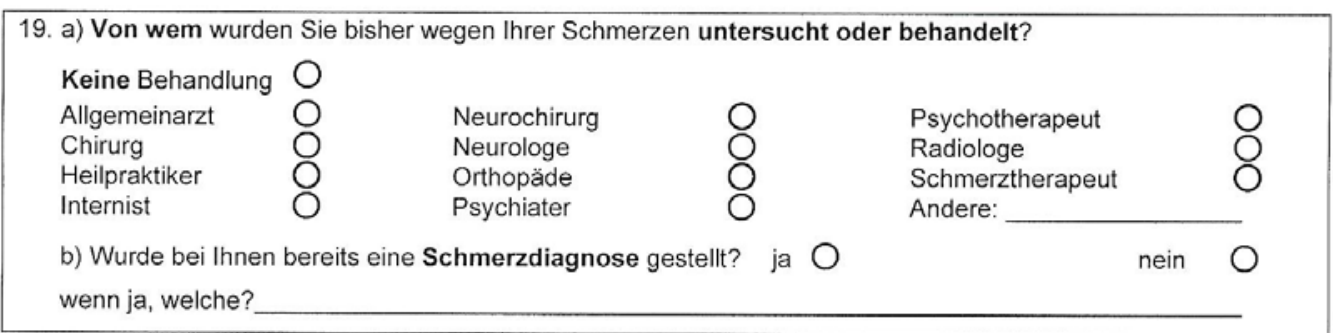

\begin{tabular}{|c|c|c|c|c|c|}
\hline \multicolumn{6}{|c|}{$\begin{array}{l}\text { 20. Wie wurden Ihre Schmerzen bisher behandelt? } \\
\text { Kreuzen Sie bitte an, welche der unten aufgeführten Behandlungsmaßnahmen Sie erhalten haben. Geben Sie } \\
\text { bitte auch an, ob Ihre Schmerzen durch diese Maßnahmen zumindest zeitweise gelindert wurden. }\end{array}$} \\
\hline & Erhalten: & Wenn ja $\rightarrow$ & ja & $\begin{array}{l}\text { wirksam? } \\
\text { voruber- } \\
\text { gehend }\end{array}$ & nein \\
\hline bisher keine Schmerzbehandlung & 0 & & & & \\
\hline Medikamente & 0 & $\rightarrow$ & 0 & 0 & 0 \\
\hline Infusionen & $\mathrm{O}$ & $\rightarrow$ & $\mathrm{O}$ & $\mathrm{O}$ & $\mathrm{O}$ \\
\hline Einspritzungen in das Schmerzgebiet, Nervenblockaden & 0 & $\rightarrow$ & $\mathrm{O}$ & $\mathrm{O}$ & 0 \\
\hline Einspritzungen am Rückenmark (z.B. epidural) & 0 & $\rightarrow$ & 0 & 0 & $\mathrm{O}$ \\
\hline Rückenmarksnahe Sonden- (SCS) oder Pumpensysteme & $\mathrm{O}$ & $\rightarrow$ & $\mathrm{O}$ & $\mathrm{O}$ & $\mathrm{O}$ \\
\hline Krankengymnastik & $\mathrm{O}$ & $\rightarrow$ & $\mathrm{O}$ & $\mathrm{O}$ & $\mathrm{O}$ \\
\hline Massagen, Bäder, Kälte-Märmetherapie & $\mathrm{O}$ & $\rightarrow$ & $\mathrm{O}$ & $\mathrm{O}$ & $\mathrm{O}$ \\
\hline Elektrische Nervenstimulation (TENS) & O & $\rightarrow$ & O & 0 & 0 \\
\hline Akupunktur & $\mathrm{O}$ & $\rightarrow$ & $\mathrm{O}$ & $\mathrm{O}$ & $\mathrm{O}$ \\
\hline Chiropraktik & O & $\rightarrow$ & $\mathrm{O}$ & $\mathrm{O}$ & $\mathrm{O}$ \\
\hline Psychotherapie & 0 & $\rightarrow$ & 0 & 0 & 0 \\
\hline Entspannungsverfahren, Hypnose, Biofeedback & $\mathrm{O}$ & $\rightarrow$ & $\mathrm{O}$ & $\mathrm{O}$ & 0 \\
\hline Medikamenten-Entzug & $\mathrm{O}$ & $\rightarrow$ & $\mathrm{O}$ & $\mathrm{O}$ & $\mathrm{O}$ \\
\hline Kur-/Reha-Behandlung & $\mathrm{O}$ & $\rightarrow$ & $\mathrm{O}$ & $\mathrm{O}$ & $\mathrm{O}$ \\
\hline Anderes: & $\mathrm{O}$ & $\rightarrow$ & $\mathrm{O}$ & 0 & 0 \\
\hline
\end{tabular}

\section{Wurden Sie schon einmal operiert?}

ja $\bigcirc \underset{\text { wio on }}{\perp} \mathrm{Lal}_{\text {mal }}$ nein $\bigcirc$

Wichtig sind für die Beantwortung dieser Frage auch alle "kleineren operativen Eingriffe", die oft in örtlicher Betäubung durchgeführt werden, z.B. Nasenoperationen, Gelenk- und Bauchspiegelungen. Bitte markieren Sie, welche Operation wegen Ihrer Schmerzen durchgeführt wurde.

Art der Operation:

Datum:

wg Schmerz $\downarrow$

1.

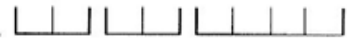

2.

3.

Lل Lل L L L

O

3.

5. 
22. Aktuelle Medikamenten-Einnahme. Bitte tragen Sie in die nachfolgende Tabelle alle Medikamente ein, die Sie zur Zeit nehmen (Schmerzmedikamente, Blutdruckmittel u.s.w)

\begin{tabular}{|l|c|c|c|c|c|c|}
\hline Beispiele & \multicolumn{3}{|c|}{$\begin{array}{c}\text { Ich nehme das Medikament } \\
\text { regelmäßig }\end{array}$} & \multicolumn{2}{c|}{$\begin{array}{c}\text { Ich nehme das Medikament } \\
\text { nur bei Bedarf }\end{array}$} \\
\hline Medikament & früh & mittags & abends & $\begin{array}{c}\text { spät } \\
\text { abends }\end{array}$ & $\begin{array}{c}\text { wenn } \\
\text { zutreffend, } \\
\text { ankreuzen }\end{array}$ & $\begin{array}{c}\text { ungefăhr wie oft } \\
\text { pro Monat? }\end{array}$ \\
\hline Beispiel: Ibuprofen 600 & $1 \mathrm{Tbl}$. & $1 \mathrm{Tbl}$. & 0 & 0 & & $\mathrm{X}$ \\
\hline Beispiel: Ibuprofen 200 & & & & & $\mathrm{Ca} .7 \mathrm{mal} 1 \mathrm{Tbl}$. \\
\hline
\end{tabular}

\begin{tabular}{|c|c|c|c|c|c|c|}
\hline \multirow[b]{2}{*}{$\begin{array}{l}\text { Bitte hier Ihre Medikamente } \\
\text { eintragen: }\end{array}$} & \multicolumn{4}{|c|}{$\begin{array}{c}\text { Ich nehme das Medikament } \\
\text { regelmäßig }\end{array}$} & \multicolumn{2}{|c|}{$\begin{array}{l}\text { Ich nehme das Medikament } \\
\text { nur bei Bedarf }\end{array}$} \\
\hline & früh & mittags & abends & $\begin{array}{c}\text { spät } \\
\text { abends }\end{array}$ & $\begin{array}{l}\text { wenn } \\
\text { zutreffend, } \\
\text { ankreuzen }\end{array}$ & $\begin{array}{l}\text { ungefähr wie oft } \\
\text { pro Monat? }\end{array}$ \\
\hline & & & & & & \\
\hline & & & & & & \\
\hline & & & & & & \\
\hline & & & & & & \\
\hline & & & & & & \\
\hline & & & & & & \\
\hline & & & & & & \\
\hline & & & & & & \\
\hline & & & & & & \\
\hline
\end{tabular}

23. Frühere Schmerzmedikamente: Bitte tragen Sie hier Ihre Schmerzmedikamente ein, die Sie früher genommen haben. Bitte bewerten Sie auch deren Wirksamkeit und eventuelle Nebenwirkungen.

\begin{tabular}{|l|l|l|l|l|}
\hline & \multicolumn{3}{|c|}{$\begin{array}{c}\text { wirksam? } \\
\text { (bitte ankreuzen) }\end{array}$} & Nebenwirkungen? \\
\hline $\begin{array}{l}\text { Ihre früheren } \\
\text { Schmerz-Medikamente }\end{array}$ & nein & etwas & ja & bitte beschreiben \\
\hline & & & & \\
\hline & & & & \\
\hline & & & & \\
\hline & & & & \\
\hline
\end{tabular}

24. Haben Sie Allergien gegen bestimmte Medikamente? wenn ja, gegen welche?

$\mathrm{jaO}$

nein $\mathrm{O}$ 


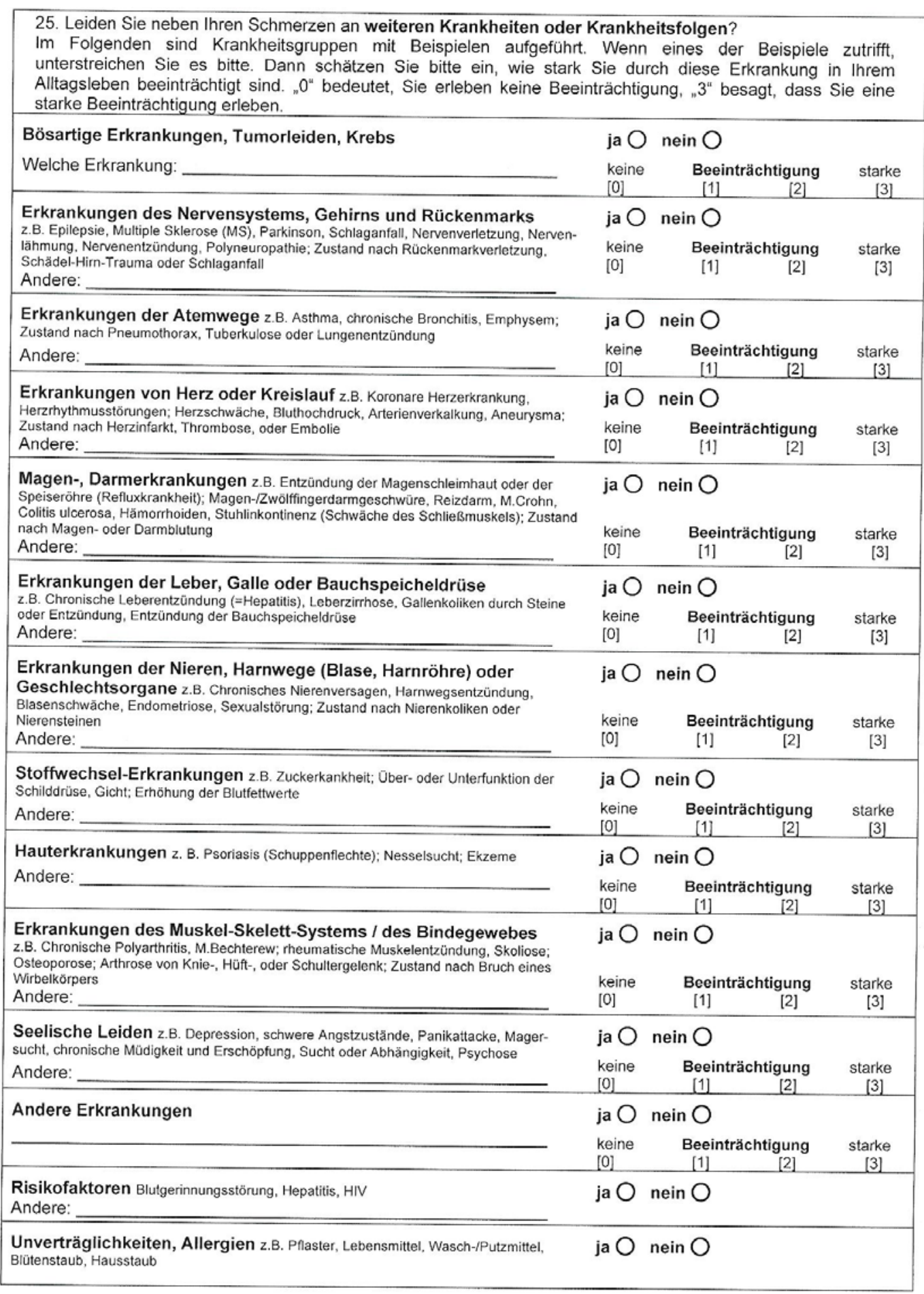




\section{Modul D Demographie, Versicherung}

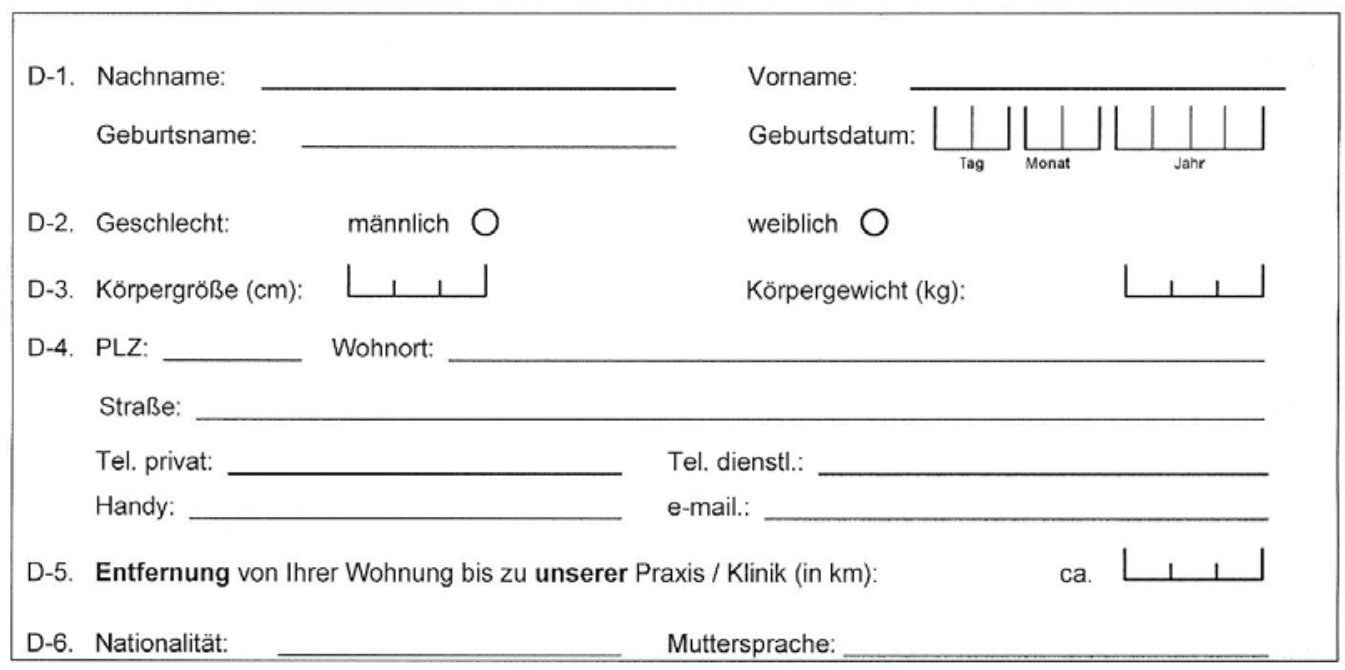

D-7. Name, Adresse und Telefon-Nummer Ihres überweisenden Arztes:

D-8. Name, Adresse und Telefon-Nummer der Ärzte oder Psychotherapeuten, die Sie zur Zeit hauptsächlich behandeln (falls abweichend von Frage D-7):

D-9. Krankenversicherung für ambulante Behandlung: zuständige Geschäftsstelle inkl. Adresse:

D-10. Krankenversicherung für stationäre Behandlung:

D-11. Beihilfe-Berechtigung: nein $\bigcirc$ ja $\bigcirc$ durch:

D-12. Behandlung aufgrund eines anerkannten BG-Verfahrens (Arbeits- oder Wegeunfall) ja $\bigcirc$ nein $O$

D-13. Haben Sie eine Krankentagegeldversicherung? ja $\mathrm{O}$ nein $\mathrm{O}$

D-14. Besteht eine Zusatzversicherung? $\quad$ ja $\bigcirc$ nein $\bigcirc$

D-15. Wer lebt gemeinsam mit Ihnen in Ihrem Haushalt? (Mehrfachantworten sind möglich):

ich lebe allein $\quad \mathrm{O}$ Ehepartner/Partner $\mathrm{O}$ Kinder $\mathrm{O}$ (Schwieger-)Eltern $\mathrm{O}$

$\begin{array}{lllll}\text { D-16. Welche Schulausbildung haben Sie? } & & \\ \text { keinen Abschluss } & \bigcirc & \text { Hauptschule / Volksschule } & O & \text { Realschule / Mittlere Reife } \\ \text { Fachhochschulreife } & \bigcirc & \text { Abitur / allgemeine Hochschulreife } & O\end{array}$




\section{Modul L Gesundheitsbezogene Lebensqualität}

Bei diesen Fragen geht es um die Beurteilung Ihres allgemeinen Gesundheitszustandes. Ihre Antworten ermöglichen, im Zeitverlauf nachzuvollziehen, wie Sie sich fühlen und wie Sie im Alltag zurechtkommen. Bitte beantworten Sie jede der folgenden Fragen, indem Sie bei den Antwortmöglichkeiten die Antwort ankreuzen, die am besten auf Sie zutrifft.

L-1. Wie würden Sie Ihren Gesundheitszustand im Allgemeinen beschreiben?

ausgezeichnet $\quad$ sehrgut $\bigcirc$ gut $\bigcirc$ weniger gut $\bigcirc$

Im. Folgenden sind einige Tätigkeiten beschrieben, die Sie vielleicht an einem normalen Tag ausüben.

Sind Sie durch Ihren derzeitigen Gesundheitszustand bei diesen Tätigkeiten eingeschränkt? Wenn ja, wie stark?

L-2. Mittelschwere Tätigkeiten, z.B. einen Tisch verschieben, staubsaugen, kegeln, Tennis spielen ja, stark eingeschränkt $\bigcirc$ ja, etwas eingeschränkt $\bigcirc \quad$ nein, überhaupt nicht eingeschränkt $\bigcirc$

L-3. Mehrere Treppenabsätze steigen ja, stark eingeschränkt $\bigcirc$

ja, etwas eingeschränkt $\bigcirc$ nein, überhaupt nicht eingeschränkt $\bigcirc$

Hatten Sie in den vergangenen 4 Wochen aufgrund Ihrer körperlichen Gesundheit irgendwelche Schwierigkeiten bei der Arbeit oder anderen alltäglichen Tätigkeiten im Beruf bzw. zu Hause?
L-4. Ich habe weniger geschafft als ich wollte.
ja $\mathrm{O}$
nein $\mathrm{O}$
L-5. Ich konnte nur bestimmte Dinge tun.
ja $\bigcirc$
nein $\mathrm{O}$

Hatten Sie in den vergangenen vier Wochen aufgrund seelischer Probleme irgendwelche Schwierigkeiten bei der Arbeit oder anderen alltäglichen Tätigkeiten im Beruf bzw. zu Hause (z.B. weil Sie sich niedergeschlagen oder ängstlich fühlten)?
L-6. Ich habe weniger geschafft als ich wollte.
ja $\mathrm{O}$
nein $\mathrm{O}$
L-7. Ich konnte nicht so sorgfältig wie üblich arbeiten.
ja $\mathrm{O}$
nein $\bigcirc$
L-8. Inwieweit haben die Schmerzen Sie in den vergangenen 4 Wochen bei der Ausübung Ihrer Alltags- tätigkeiten zuhause und im Beruf behindert?
überhaupt nicht $\bigcirc$ ein bisschen mäßig $\mathrm{O}$ ziemlich $\mathrm{O}$ sehr $\mathrm{O}$

In diesen Fragen geht es darum, wie Sie sich fühlen und wie es Ihnen in den vergangenen 4 Wochen gegangen ist (bitte kreuzen Sie in jeder Zeile den Begriff an, der Ihrem Befinden am ehesten entspricht).

Wie oft waren Sie in den vergangenen 4 Wochen ...

L-9. ruhig und gelassen...

manchmal $\mathrm{O}$

selten $\mathrm{O}$

nie

L-10. voller Energie...

immer $\mathrm{O}$

meistens $\bigcirc \quad$ ziemlich $\bigcirc$

manchmal $\bigcirc$

selten $\mathrm{O}$ nie $\mathrm{O}$

$\mathrm{L}-11$. entmutigt und traurig...
immer $\mathrm{O}$
meistens $\mathrm{O}$
ziemlich $\mathrm{O}$
manchmal $\mathrm{O}$
selten $\mathrm{O}$
nie

L-12. Wie häufig haben Ihre körperliche Gesundheit oder seelischen Probleme in den vergangenen 4 Wochen Ihre Kontakte zu anderen Menschen (Besuche bei Freunden, Bekannten, usw.) beeinträchtigt?

immer $\mathrm{O}$ meistens $\mathrm{O}$

manchmal $\bigcirc$

selten

nie 


\section{Modul S Sozialrechtliche Situation}

S-1. Sind Sie zur Zeit berufstätig? (trifft auch zu, wenn Sie jetzt gerade arbeitsunfähig sind) ja, ich bin zur Zeit berufstätig, d.h. habe einen Arbeitsplatz nein, ich bin zur Zeit nicht berufstätig

$\rightarrow$ weiter bei Frage S-2

$\rightarrow$ weiter bei Frage S-5

Die Fragen S-2 bis S-4 bitte nur beantworten, wenn Sie zur Zeit berufstätig sind.

S-2. Welche berufliche Tätigkeit üben Sie aus?

S-3. Sind Sie zur Zeit arbeitsunfähig?

ja $\mathrm{O}$ nein $\mathrm{O}$

wenn ja, glauben Sie, dass Sie wieder an Ihren alten Arbeitsplatz

zurückkehren können?

ja $\mathrm{O}$ nein $\mathrm{O}$

S-4. Wie viele Tage waren Sie in den letzten 3 Monaten arbeitsunfähig?

ich war in letzten 3 Monaten an Tagen arbeitsunfähig (0-92 Tage)

bin durchgehend arbeitsunfähig $\bigcirc$ seit

$$
\text { Taq } \underset{\text { Monat }}{\text { Jahr }}
$$

Die Frage S-5 bitte nur beantworten, wenn Sie derzeit nicht berufstätig sind.

S-5. Wenn Sie zur Zeit nicht berufstätig sind, sind Sie ...

$\begin{array}{llll}\text { Schüler/in, Student/in } \mathrm{O} & \text { Hausfrau / Hausmann } & \text { berentet } \mathrm{O} \\ \text { arbeitslos / erwerbslos } \mathrm{O} & \text { seit__Lnat/Jahr) } & \end{array}$

S-6. Beabsichtigen Sie einen Renten-Antrag oder einen Antrag auf Renten-Änderung zu stellen?

ja $\mathrm{O}$ nein $\mathrm{O}$

Haben Sie einen Rentenantrag / Antrag auf Renten-Änderung gestellt, der noch nicht entschieden ist? nein $\mathrm{O}$

ja $\mathrm{O}$

wenn ja, wegen:

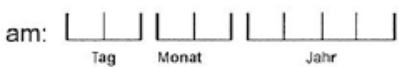

Ist bereits ein Rentenantrag abgelehnt worden?

ja $\bigcirc$ nein $\mathrm{O}$

Befindet sich derzeit ein Rentenantrag im Widerspruchsverfahren? ja $\bigcirc$ nein $\bigcirc$

S-7. Beziehen Sie derzeit eine Rente? ja $\mathrm{O}$ nein $\mathrm{O}$

wenn ja, auf Zeit $\bigcirc$ bis wann: endgültig $\bigcirc$ seit wann:

wenn ja, Berentung welcher Art? vorgezogenes Altersruhegeld

Berufsunfähigkeit

$\mathrm{O}$

Erwerbsunfähigkeit

O

Erreichen der Altersgrenze

O

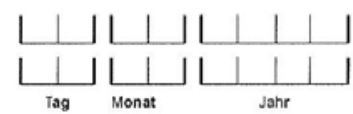

Teilweise Erwerbsminderung Volle Erwerbsminderung Unfallrente

Witwen- oder Waisenrente

S-8. Haben Sie einen (z.B. durch das Amt für Versorgungsangelegenheiten) anerkannten Grad der Behinderung? (GdB) ja $\mathrm{O}$ nein $\mathrm{O} \rightarrow$ wenn ja, wie hoch ist der GdB? Ist ein GdB oder eine Höherstufung beantragt?

ja $\mathrm{O}$ nein $\mathrm{O}$ 
Modul V

\section{Vorbehandlungen}

V-1 Bitte notieren Sie soweit möglich jeweils Namen, Fachrichtung und Adresse von Ärzten, Heilpraktikern und anderen Therapeuten, bei denen Sie wegen Ihrer Schmerzen bereits in Behandlung waren oder sind.

Name

Fachrichtung

Adresse

in Behandlung

von - bis

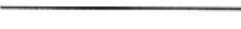
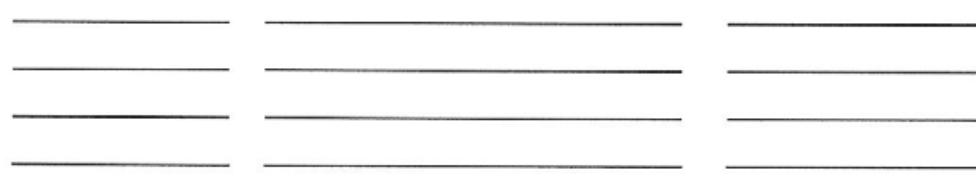

V-2 Bitte notieren Sie hier Ihre schmerzbezogenen stationären Behandlungen (Krankenhausaufenthalte) Name
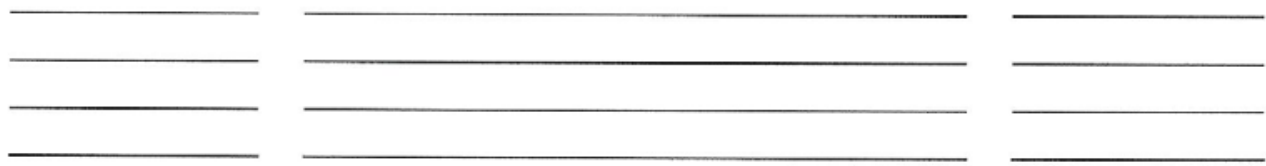

V-3 Bitte notieren Sie hier Ihre schmerzbezogenen Aufenthalte in Kur- oder Rehabilitationskliniken Name

Adresse

von - bis

Wir benötigen zur vollständigen Erfassung Ihrer Krankheitsgeschichte evtl. die Befunde der aufgeführten ärztlichen Kollegen bzw. der aufgeführten therapeutischen Einrichtungen.

Die Befunde dürfen wir nur mit Ihrem ausdrücklichen Einverständnis anfordern. Wir bitten Sie daher, die nachfolgende so genannte Entbindung von der Schweigepflicht zu unterschreiben.

\section{Entbindung von der Schweigepflicht}

Ich bin mit der Anforderung von Unterlagen, die der Vervollständigung meiner Krankheitsgeschichte dienen, durch die

\section{Stempelfeld}

einverstanden. 
Modul A

Allgemeinbefindlichkeit

Die folgenden Fragen beziehen sich auf die letzten 14 Tage:

A-1. Wie war Ihr allgemeines Wohlbefinden? Ordnen Sie Ihrem Befinden eine Position auf der Linie zu, wobe ${ }_{n}-100 "$ einem sehr schlechten Befinden und ${ }_{n}+100 "$ einem sehr guten Befinden entspricht. Machen Sie eine Markierung an der Stelle, die Ihrem Befinden entspricht.

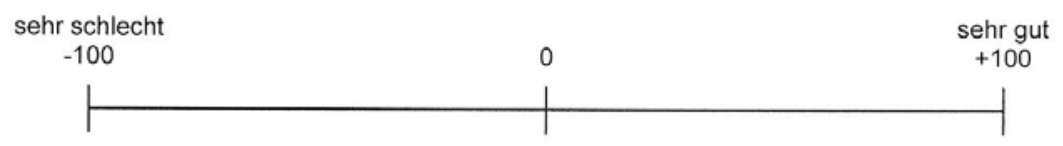

A-2. War Ihre nächtliche Schlafdauer...

ausreichend? ..nicht ausreichend?

A-3. Hatten Sie Dauerschmerzen:

nein $\bigcirc$

ja $\bigcirc$

A-4. Wurden Sie durch Ihre Schmerzen in Ihren Tätigkeiten und Bedürfnissen eingeschränkt?

nein $\mathrm{O}$

ja, ein wenig $\bigcirc$

deutlich $\mathrm{O}$

stark $\bigcirc$

fast völlig $\mathrm{O}$

A-5. Haben die Schmerzen Ihre Stimmung beeinträchtigt?

nein $\mathrm{O}$

ja, ein wenig $\bigcirc$

deutlich $\bigcirc$

stark $\bigcirc$

sehr stark $\bigcirc$

A-6. Hatten Sie das Gefühl, die Schmerzen lindernd beeinflussen zu können?

nein $\mathrm{O}$

ja, ein wenig $\mathrm{O}$

deutlich

stark $\mathrm{O}$

sehr stark

A-7. Hatten Sie sonstige Beschwerden? (Mehrfachnennungen möglich)

keine

O

Müdigkeit

O

Übelkeit

0

Magenbeschwerden $\mathrm{O}$

Konzentrationsstörung $\mathrm{O}$

Niedergeschlagenheit

Appetitlosigkeit

Schlafstörungen

Schwitzen

$\begin{array}{ll}\text { Lustlosigkeit } & \bigcirc \\ \text { Schwindel } & \bigcirc \\ \text { Verstopfung } & \bigcirc\end{array}$

Andere 


\section{Danksagung}

Ich danke allen Patienten der Schmerz-Tagesklinik Göttingen, die sich bereit erklärt haben, an meiner Befragung teilzunehmen. Ohne sie wäre die Durchführung der Arbeit nicht möglich gewesen.

Bedanken möchte ich mich außerdem bei allen Mitarbeitern der Schmerz-Tagesklinik und -ambulanz Göttingen, die stets bemüht waren, mir bei der Durchführung des Projektes zu helfen.

Außerdem danke ich Frau Prof. Hummers-Pradier für die kritische Korrektur meiner Arbeit.

Mein besonderer Dank gilt Herrn Prof. Dr. phil. Dipl.-Psych. Michael Pfingsten, der mir während der letzten Jahre jederzeit unterstützend zur Seite stand, mich stets ermutigte und mir die Möglichkeit gab, meine Ergebnisse auf dem Deutschen Schmerzkongress 2010 in Mannheim zu präsentieren. 